\title{
Single-Shot Error Correction of Three-Dimensional Homological Product Codes
}

\author{
Armanda O. Quintavalle $\odot,{ }^{1, *}$ Michael Vasmer $\odot,{ }^{2,3, \dagger}$ Joschka Roffe $\odot,{ }^{1,4, \sharp}$ and Earl T. Campbell ${ }^{1,5, \S}$ \\ ${ }^{1}$ Department of Physics and Astronomy, University of Sheffield, Sheffield S3 7RH, United Kingdom \\ ${ }^{2}$ Perimeter Institute for Theoretical Physics, Waterloo, Ontario N2L 2Y5, Canada \\ ${ }^{3}$ Institute for Quantum Computing, University of Waterloo, Waterloo, Ontario N2L 3G1, Canada \\ ${ }^{4}$ Dahlem Center for Complex Quantum Systems, Freie Universität Berlin, 14195 Berlin, Germany \\ 5 AWS Center for Quantum Computing, Cambridge, CB1 2GA, United Kingdom
}

(Received 17 December 2020; accepted 20 April 2021; published 14 June 2021)

\begin{abstract}
Single-shot error correction corrects data noise using only a single round of noisy measurements on the data qubits, removing the need for intensive measurement repetition. We introduce a general concept of confinement for quantum codes, which roughly stipulates qubit errors cannot grow without triggering more measurement syndromes. We prove confinement is sufficient for single-shot decoding of adversarial errors and linear confinement is sufficient for single-shot decoding of local stochastic errors. Further to this, we prove that all three-dimensional homological product codes exhibit confinement in their $X$ components and are therefore single shot for adversarial phase-flip noise. For local stochastic phase-flip noise, we numerically explore these codes and again find evidence of single-shot protection. Our Monte Carlo simulations indicate sustainable thresholds of 3.08(4)\% and 2.90(2)\% for three-dimensional (3D) surface and toric codes, respectively, the highest observed single-shot thresholds to date. To demonstrate single-shot error correction beyond the class of topological codes, we also run simulations on a randomly constructed family of 3D homological product codes.
\end{abstract}

DOI: 10.1103/PRXQuantum.2.020340

\section{INTRODUCTION}

Quantum error correction encodes logical quantum information into a codespace [1]. Given perfect measurement of the codespace stabilizers we obtain the syndrome of any error present. A suitable decoding algorithm can determine a recovery operation that returns the system to the codespace. Either this recovery is a perfect success, or a failure resulting in a high weight logical error. However, in real quantum systems the measurements are not perfect and this simple story becomes more involved. The three main strategies for tackling noisy measurements are as follows: repeated measurements on the code $[2,3]$; performing measurement driven error correction on a cluster state [4-9]; or using a single-shot code and decoder [10]. Focusing on the last strategy, the single-shot approach has the advantage of

\footnotetext{
*armandaoq@gmail.com

†mvasmer@perimeterinstitute.ca

¥joschka@roffe.eu

\$earltcampbell@gmail.com
}

Published by the American Physical Society under the terms of the Creative Commons Attribution 4.0 International license. Further distribution of this work must maintain attribution to the author(s) and the published article's title, journal citation, and DOI. no additional time cost or cluster-state generation cost and provides a resilience against time-correlated noise [11]. In single-shot error correction, some residual error persists after each round of error correction, but this residual error is kept small and does not rapidly accumulate. However, only a special class of codes support single-shot error correction, but exactly which codes and why is not yet fully understood.

Bombin coined the phrase single-shot error correction and remarked that it "is related to self-correction and confinement phenomena in the corresponding quantum Hamiltonian model." [10]. He defined confinement for subsystem codes, and showed that it is sufficient for singleshot error correction with a limited class of subsystem codes. In particular, he proved that the three-dimensional (3D) gauge color code supports single-shot error correction, though it is unknown whether the corresponding Hamiltonian exhibits self-correction. Later single-shot error correction was numerically observed in a variety of higher-dimensional topological codes, including the following: the 3D gauge color code [12], four-dimensional (4D) surface codes [13] and their hyperbolic cousins [14], and 3D surface codes with phase noise [15-17]. Campbell established a general set of sufficient conditions, encapsulated by a code property called good soundness, that ensured adversarial noise could be suppressed using a 
single-shot decoder [18]. While Campbell's sufficiency conditions explained single-shot error correction in a wide range of codes, around the same time it was shown that quantum expander codes [19-21] supported single-shot error correction [22]. However, quantum expander codes lack the soundness property so neither Bombín's notion of confinement or Campbell's notion of soundness is sufficient to encompass all known examples of single-shot error correction. Our work provides the first framework that captures all known forms of single-shot error correction, encompassing both previous approaches within a single theory.

We can use different classical algorithms to decode a given quantum code, and this choice will affect the utility of the code. Different decoders have various time complexities and error tolerances, which affects the resources required by a quantum computer based on the code [23-25]. Thus far, single-shot decoders come in two flavors. The first are two-stage decoders [12,13], where stage 1 decoding repairs the noisy syndrome using redundancy in the parity check measurements and stage 2 decoding solves the corrected syndrome problem. The second flavor of decoders computes a correction from the noisy syndrome without attempting to repair it. Most examples of such decoders are local decoders, meaning that the whole correction is made up of corrections computed in small local regions of the code using syndrome information in the immediate neighborhood [14-17,21,26,27]. However, there are some examples of nonlocal decoders such as belief propagation (BP) being used for single-shot error correction without syndrome repair [14,27]. A natural question to ask is the following: what is the optimal decoding strategy for single-shot codes? Even in the simple case of the 3D toric code this is not well understood.

The remainder of this paper is structured as follows. In Sec. II, we give a summary of our results. In Sec. III, we formally state our results on confinement and singleshot decoding. In Sec. IV, we detail the construction of 3D product codes. In Sec. V, we present our numerical simulations and analyse their results. Finally, in Sec. VI, we discuss future research directions that flow from this work.

\section{SUMMARY OF RESULTS}

This paper is in two parts: on the one hand, we propose the concept of confinement as an essential characteristic for a code family to display single-shot properties; on the other, we investigate the single-shot performances of the class of 3D homological product codes [19,28,29], which we call 3D product codes. First, we introduce confinement in Sec. III. Loosely, confinement stipulates that syndrome weight must increase with qubit weight, under some caveats. We formalize the notion of a code family having good confinement, which we prove is a sufficient condition for single-shot decoding in the adversarial noise setting. In addition to that, we prove that good linear confinement is a sufficient condition for a family of codes to exhibit a sustainable single-shot threshold for local stochastic noise (Appendix A). Second, we review the construction of the 3D product codes in Sec. IV, and show that the 3D surface and toric codes are particular instances of this more general class of codes in Appendix B. We prove that all 3D product codes have (cubic) confinement for phase-flip errors (Appendix C), and therefore have singleshot error correction for adversarial phase-flip noise. We expect these codes to have single-shot error correction for local stochastic phase-flip noise as well. In fact, our definition of confinement generalizes the definition proposed by Bombín [10] for the gauge color code and the notion of robustness for expander codes [20]; since both class of codes are proven to have a single-shot threshold for local stochastic noise $[10,22]$ we conjecture that low-density parity-check (LDPC) codes with good (superlinear) confinement have a threshold too. We investigate this case numerically.

In the single-shot setting, the code always has some residual error present and the error-correction procedure introduces noise correlations in subsequent rounds of single-shot error correction. How then do we assess success or failure of a decoding algorithm? The concept of sustainable threshold was proposed by Brown et al. [12] as a metric for single-shot codes and decoders. We use $p_{\text {th }}(N)$ to denote the threshold of a code-decoder family given $N$ cycles of qubit noise, noisy syndrome extraction, and single-shot decoding, with the $N^{\text {th }}$ cycle followed by a single round of noiseless syndrome extraction and decoding. The final round ensures that we return the system to the codespace and assess success by the absence of a logical error. We define the sustainable threshold of the code-decoder family to be

$$
p_{\text {sus }}=\lim _{N \rightarrow \infty} p_{\text {th }}(N) .
$$

Numerically, this is estimated by plotting $p_{\text {th }}(N)$ against $N$ and fitting to the following ansatz,

$$
p_{\text {th }}(N)=p_{\text {sus }}\left\{1-\left[1-p_{\text {th }}(0) / p_{\text {sus }}\right] e^{-\gamma N}\right\} .
$$

We numerically estimate the sustainable error thresholds of 3D toric and surface codes for two different two-stage decoders. We surpass all previous single-shot error thresholds for these code families, and we also obtain the highest phase-flip noise threshold; see Table I. For our singleshot simulations, we use an independent and identically distributed noise model where each qubit experiences a phase-flip error with probability $p$, and each stabilizer measurement outcome is flipped with probability $q=p$. We investigate two decoding strategies: one where we use minimum-weight perfect matching (MWPM) for stage-1 decoding and belief propagation with ordered statistics 
TABLE I. Comparison of the error thresholds of toric code decoders (results from this work are highlighted in bold). For phase-flip noise, BP+OSD outperforms all prior art, and approaches the theoretical upper bound given by mapping to a statistical mechanical model. In the single-shot regime, MWPM and BP+OSD outperforms the Sweep decoder (the theoretical maximum is unknown in this case).

\begin{tabular}{lc}
\hline \hline Toric code decoder & Phase-flip threshold \\
\hline Erasure mapping [30] & $12.2 \%$ \\
Toom's rule [15] & $14.5 \%$ \\
Sweep [17] & $15.5 \%$ \\
Renormalization group [13] & $17.2 \%$ \\
Neural network [31] & $17.5 \%$ \\
BP+OSD & $\mathbf{2 1 . 5 5 ( 1 ) \%}$ \\
Statistical phase transition [32-36] & $23.180(4) \%$ \\
\hline & Single-shot threshold \\
\hline Sweep & $1.7 \%$ \\
MWPM and BP+OSD & $\mathbf{2 . 9 0}(\mathbf{2}) \%$ \\
\hline \hline
\end{tabular}

decoding $(\mathrm{BP}+\mathrm{OSD})$ for stage-2 decoding and another where we use BP+OSD for both decoding stages. Figure 1 shows the 3D surface code sustainable threshold fit, using the MWPM and BP+OSD decoding strategy. We find a comparable sustainable threshold for the 3D surface code using $\mathrm{BP}+\mathrm{OSD}$ for both decoding stages, as shown in Table II.

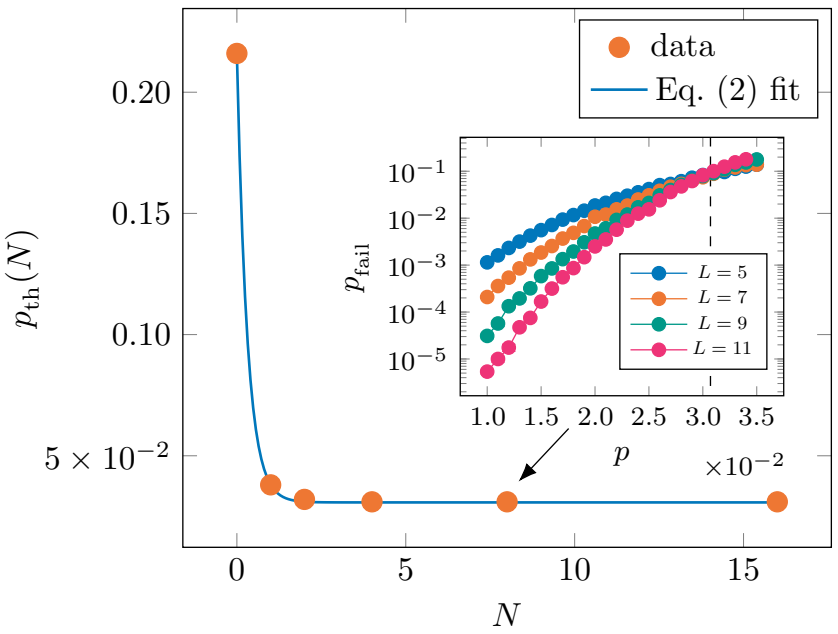

FIG. 1. Numerical estimate of the sustainable threshold of the 3D surface code for a two-stage decoder where we repair the syndrome using MWPM, and solve the corrected syndrome problem using $\mathrm{BP}+\mathrm{OSD}$. We plot the errors threshold $p_{\text {th }}(N)$ for different numbers of cycles, $N$. Using the ansatz in Eq. (2), we estimate the sustainable threshold to be $p_{\text {sus }}=0.0308$ (4) with $\gamma=3.23$. The inset shows a plot of the logical error rate, $p_{\text {fail }}$, against the phase-flip and measurement error rate, $p$, for $N=8$. The error threshold $p_{\text {th }}(8)$ is the point at which the curves intersect ( $L$ is the code distance).
TABLE II. Sustainable thresholds for 3D toric and surface codes for different single-shot decoding strategies. For each entry in the table, we did an analogous simulation to that described in Fig. 1. The numbers in brackets are the standard errors.

\begin{tabular}{lcc}
\hline \hline Code & MWPM and BP+OSD & BP+OSD $\times 2$ \\
\hline Surface & $3.08(4) \%$ & $2.90(1) \%$ \\
Toric & $2.90(2) \%$ & $2.78(2) \%$ \\
\hline \hline
\end{tabular}

There is an important difference in single-shot decoding for the 3D toric code when compared with the 3D surface code. Specifically, in the 3D toric code, the syndromerepair stage of single-shot decoding can fail, producing a "syndrome" for which there is no corresponding error. We find that this failure mode substantially increases the logical error rate of the 3D toric code when compared with the 3D surface code (although the thresholds are very similar). We provide a novel decoding subroutine for dealing with these errors, which dramatically improves the performance of the 3D toric code. Furthermore, our subroutine is applicable to any single-shot LDPC code whose parity-check matrix is not full rank.

The advantage of using BP+OSD for stage- 1 decoding is that, unlike MWPM, this decoder does not rely on the special structure of the looplike syndrome present in 3D toric and surface codes. We use BP+OSD for single-shot decoding of a family of nontopological 3D product codes, achieving sustainable thresholds that are comparable to those of the 3D surface code. This is the second example of single-shot decoding of nontopological codes, the first being the quantum expander codes considered in Ref. [27]. Whilst our single-shot threshold $(2.7 \%)$ is slightly below the corresponding value observed in quantum expander codes $(3 \%)$, our code family has other advantages over expander codes. Most importantly, the expansion properties of our code family are less severe, which implies that our code family would be easier to implement in architectures with geometrically constrained connectivity.

\section{DEFINITIONS AND THEOREMS}

In this section, we introduce the definition of confinement for a stabilizer code and exhibit a theoretical twostage decoder, the shadow decoder, which we prove is single shot on confined codes against adversarial noise. We refer the reader to Appendix A to see how a variant of the shadow decoder can be used to prove that good families of codes with linear confinement have a single-shot threshold for local stochastic noise.

A stabilizer code encoding $k$ logical qubits into $n$ physical qubits can be described by its stabilizer group $\mathcal{S}$ and a syndrome map $\sigma(\cdot)$. The stabilizer group $\mathcal{S}$ is an Abelian subgroup of the Pauli group $\mathcal{P}_{n}$ on $n$ qubits, which does not contain $-\mathbb{1}$ and has dimension $n-k$. The syndrome map is not unique: any generating set of the group $\mathcal{S}$ defines a 
valid syndrome map for the code. If $\left\{s_{1}, \ldots, s_{m}\right\}$ is one such generating set, the associated function $\sigma(\cdot)$ maps a qubit operator $p \in \mathcal{P}_{n}$ into the binary vector $\left(\bar{s}_{1}, \ldots, \bar{s}_{m}\right)^{T} \in \mathbb{F}_{2}^{m}$, where $\bar{s}_{i}=1$ if $s_{i}$ anticommutes with $p$ and 0 otherwise. Importantly, $\sigma(\cdot)$ is linear, meaning that $\sigma(p \cdot q)=$ $\sigma(p)+\sigma(q)$ over $\mathbb{F}_{2}^{m}$. Because any Pauli operator $p \in \mathcal{P}_{n}$ can be factorized as the product of an $X$ and a $Z$ operator $p_{X}$ and $p_{Z}$, we can identify it with a binary vector $\bar{p}=\left(\bar{p}_{X}, \bar{p}_{Z}\right)^{T} \in \mathbb{F}_{2}^{2 n}$, where the $i$ th entry of $\bar{p}_{X} / \bar{p}_{Z}$ is 1 if and only if $p_{X} / p_{Z}$ acts nontrivially on the $i$ th qubit. Given a Pauli operator $p$, its weight $|p|$ is the number of qubits on which its action is not the identity. Consider a stabilizer code with syndrome function $\sigma(\cdot)$, then the reduced weight of a Pauli operator $p \in \mathcal{P}_{n}$ on the physical qubits is

$$
|p|^{\text {red }}:=\min \left\{|q|: \sigma(q)=\sigma(p), q \in \mathcal{P}_{n}\right\} .
$$

A stabilizer code is said to be distance $d$ if $d$ is the minimum weight of a Pauli operator not in $\mathcal{S}$ that has trivial syndrome. We refer to a code of length $n$, dimension $k$, and distance $d$ as a $[[n, k, d]]$ code.

For a stabilizer code, we then have the following.

Definition 1 (Confinement). Let $t$ be an integer and $f$ : $\mathbb{Z} \rightarrow \mathbb{R}$ some increasing function with $f(0)=0$. We say that a stabilizer code has $(t, f)$ confinement if, for all errors $e$ with $|e|^{\text {red }} \leq t$, it holds

$$
f(|\sigma(e)|) \geq|e|^{\text {red }} .
$$

The reduced weight of operators is crucial in this definition to avoid making confinement fail by adding stabilizer operators to arbitrarily increase the weight of an otherwise low weight error.

Let us contrast this with Bombín's notion of confinement (Definition 16 of Ref. [10]) that has some similarities but allows only for linear functions of the form $f(x)=\kappa x$ for some constant $\kappa$. Many codes, including $3 \mathrm{D}$ product codes, have superlinear confinement functions, as such Bombín's definition does not encompass them. Moreover, the concept of confinement is closely related to soundness [18] but it is weaker and so able to encompass more families of codes, such as the expander codes [19-21], which are confined but not sound. Roughly speaking, a code has good confinement if small qubit errors produce small measurement syndromes; this differs from good soundness, which entails that small syndromes can be produced by small errors.

Formally, we define the following notion of good confinement for a family of stabilizer codes.

Definition 2 (Good confinement). Consider an infinite family of stabilizer codes. We say that the family has good confinement if each code in it has $(t, f)$ confinement, where the following holds:
1. t grows with the length $n$ of the code: $t \in \Omega\left(n^{b}\right)$ with $b>0$;

2. and $f(\cdot)$ is monotonically increasing and independent of $n$.

We say the code family has good $X$ confinement if the above holds only for Pauli-Z errors.

Our main analytic result is that codes with good confinement are single shot.

Theorem 1. Consider a family of $[[n, k, d]]$ quantumLDPC codes with good confinement and growing distance $d \geq a n^{b}$ with $a>0$ and $b>0$. This code family is single shot for the adversarial noise model. If the code family only has good $X$ confinement then it is single shot with respect to Pauli-Z noise.

We conjecture that the result of Theorem 1 can be extended to deal with local stochastic noise and used to show that LDPC codes with good confinement have a single-shot threshold. In this direction, we are able to prove that linear confinement is sufficient for codes to exhibit a single-shot threshold in the local stochastic noise setting.

Theorem 2. Consider a family of $[[n, k, d]]$ quantumLDPC codes with qubit degree at most $\omega-1$ and good linear confinement such that $d \geq a n^{b}$ with $a>0$ and $b>$ 0 . This code family has a sustainable single-shot threshold for any local stochastic noise model. If the code family only has good $X$ confinement then it has a sustainable single-shot threshold with respect to Pauli-Z noise.

We further prove that 3D product codes have $X$ confinement.

Theorem 3. All $3 D$ product codes have $(t, f) X$ confinement, where $t$ is equal to the $Z$ distance of the code and $f(x)=x^{3} / 4$ or better .

Theorems 1 and 3 together motivate our numerical experiments reported in Sec. V.

We now proceed to prove Theorem 1. To this end, we use the shadow decoder that we introduce in Definition 3. The shadow decoder differs from previous single-shot two stage decoders (e.g., the MW single-shot decoder introduced in Definition 6 of [18]) in that it does not rely on metachecks on syndromes. If syndromes are protected by a classical code, as is the case for $X$ syndromes of 3D product codes introduced in Sec. IV, then a single-shot decoding strategy could work as follows: (1) correct the measured syndrome whenever it does not satisfy all the constraints defined by the metacode; (2) find a recovery operator on qubits that has syndrome equal to the one found at point (1). The shadow decoder, instead, corrects 
the syndrome both anytime it fails to satisfy all the constraints of the metacode and when it is generated by high weight errors. We do not describe how to implement it or make statements concerning the complexity of decoding. Our proof makes similar assumptions as the KovalevPryadko quantum-LDPC threshold theorem [37] where they assumed a minimum-weight decoder without addressing implementation issues. Indeed, decoding for arbitrary LDPC codes is an nondeterministic polynomial-time complete (NP complete) problem that we do not expect to be efficiently solvable in full generality.

The building blocks of the shadow decoder are the $t$ shadows of the code. A $t$ shadow is a set in the syndrome space, which contains all the images of Pauli errors $e$ on the physical qubits that have weight at most $t$. In other words, if we identify Pauli errors $e$ on $n$ qubits with $2 n$-bit strings and we consider the metric space $\mathcal{M}=\mathbb{F}_{2}^{2 n}$ endowed with the Hamming distance [i.e., the distance $d\left(\bar{e}_{1}, \bar{e}_{2}\right)$ between the vectors $\bar{e}_{1}$ and $\bar{e}_{2}$ corresponding to the Pauli errors $e_{1}$ and $e_{2}$, respectively, is defined as $\left.d\left(\bar{e}_{1}, \bar{e}_{2}\right)=\left|e_{1}+e_{2}\right|\right]$ then the $t$ shadow of the code is the image, via the syndrome function $\sigma(\cdot)$, of the ball of radius $t$ centered at 0 in $\mathcal{M}$. Note that, because balls on $\mathcal{M}$ are not vector spaces, the shadows are not vector spaces either.

We are now ready to introduce the shadow decoder.

Definition 3 (Shadow decoder). The shadow decoder has variable parameter $t>0$. Given an observed syndrome $\bar{s}=\sigma(e)+\bar{s}_{e}$ where $\bar{s}_{e} \in \mathbb{F}_{2}^{m}$ is the syndrome error, the shadow decoder of parameter t performs the following two steps:

1. Syndrome repair: find a binary vector $\bar{s}_{r}$ of minimum weight $\left|\bar{s}_{r}\right|$ such that $\bar{s}+\bar{s}_{r}$ belongs to the $t$ shadow of the code, where

$$
t \text { shadow }=\{\sigma(e):|e| \leq t\} .
$$

2. Qubit decode: find $e_{r}$ of minimum weight $\left|e_{r}\right|$ such that $\sigma\left(e_{r}\right)=\bar{s}+\bar{s}_{r}$.

We call $r=e_{r} \cdot e$ the residual error.

A key result in proving Theorem 1 is the following promise on the performance of the shadow decoder: when a code has confinement, the weight of the residual error after one decoding cycle is bounded by a function of the weight of the syndrome error.

Lemma 1. Consider a stabilizer code that has $(t, f)$ confinement. Provided that the original error pattern e has $|e|^{\text {red }} \leq t / 2$, on input of the observed syndrome $\bar{s}=\sigma(e)+$ $\bar{s}_{e}$, the residual error $r$ left by the shadow decoder of parameter $t / 2$ satisfies

$$
|r|^{\text {red }} \leq f\left(2\left|\bar{s}_{e}\right|\right)
$$

Assume $|e|^{\text {red }} \leq t / 2$. By construction, $e_{r}$ has minimum weight among all errors with syndrome $\sigma(e)+\bar{s}_{e}+\bar{s}_{r} \in t / 2$ shadow of the code. In particular, $\left|e_{r}\right| \leq t / 2$. By the triangular inequality for the weight function,

$$
\left|e_{r} \cdot e\right|^{\text {red }} \leq\left|e_{r}\right|^{\text {red }}+|e|^{\text {red }} \leq t .
$$

Therefore, we can apply the confinement property on the residual error $r=e_{r} \cdot e$ :

$$
f\left(\left|\sigma\left(e_{r} \cdot e\right)\right|\right) \geq\left|e_{r} \cdot e\right|^{\mathrm{red}} .
$$

By linearity of the syndrome function $\sigma(\cdot)$ :

$$
\sigma\left(e_{r} \cdot e\right)=\sigma\left(e_{r}\right)+\sigma(e)=\bar{s}_{e}+\bar{s}_{r} .
$$

Note that the syndrome error $\bar{s}_{e}$ is a possible solution of the syndrome repair step of the shadow decoder, because by assumption $|e|^{\text {red }} \leq t / 2$. Thus, $\left|\bar{s}_{r}\right| \leq\left|\bar{s}_{e}\right|$ and

$$
\left|\bar{s}_{e}+\bar{s}_{r}\right| \leq\left|\bar{s}_{e}\right|+\left|\bar{s}_{r}\right| \leq 2\left|\bar{s}_{e}\right| .
$$

Combining these and the monotonicity of $f$ leads to the required bound on the residual error $r=e_{r} \cdot e$ :

$$
\left|e_{r} \cdot e\right|^{\text {red }} \leq f\left(2\left|\bar{s}_{e}\right|\right) .
$$

Theorem 1 follows directly from Lemma 1 . In particular, Lemma 1 entails that a code with $(t, f)$ confinement is robust against $N$ cycles of qubit noise, noisy syndrome extraction, and single-shot decoding, as explained below.

At each cycle $\tau$, we assume that a new error $e^{\tau}$ is introduced in the system and it is added to the residual error $r^{\tau-1}$. We assume that for the new physical error $e^{\tau}$ and the syndrome measurement error $\bar{s}_{e}^{\tau}$ the following hold:

$$
\left|e^{\tau}\right|^{\mathrm{red}} \leq t / 4 \text { and } f\left(2\left|\bar{s}_{e}^{\tau}\right|\right) \leq t / 4 .
$$

We perform syndrome extraction on the state $\hat{e}^{\tau}=e^{\tau}$. $r^{\tau-1}$. The noisy syndrome $\bar{s}^{\tau}=\sigma\left(\hat{e}^{\tau}\right)+\bar{s}_{e}^{\tau}$ is used as input for the shadow decoder of parameter $t / 2$. The recovery operator $e_{r}^{\tau}$ found by the shadow decoder is then applied to the state and finally a new cycle starts where $r^{\tau}=e_{r}^{\tau} \cdot \hat{e}^{\tau}$. Let $r^{0}=\mathbb{1}$, so that the initial state of the system is given by $\hat{e}^{1}=e^{1}, \bar{s}^{1}=\sigma\left(\hat{e}^{1}\right)+\bar{s}_{e}^{1}$. Note that if

$$
\left|e^{\tau}\right|^{\text {red }},\left|r^{\tau-1}\right|^{\text {red }} \leq t / 4
$$

then $\left|\hat{e}^{\tau}\right|^{\text {red }}=\left|e^{\tau} \cdot r^{\tau-1}\right|^{\text {red }} \leq t / 2$ and the hypotheses of Lemma 1. Combining this with the bound on the syndrome error, Eq. (9), we obtain

$$
\left|r^{\tau}\right|^{\text {red }} \leq f\left(2\left|\bar{s}_{e}^{\tau}\right|\right) \leq \frac{t}{4} .
$$

In conclusion, provided that the conditions on the physical and the measurement errors, Eq. (9), are satisfied for each 
iteration up to $\tau-1$, the residual error after the $\tau^{\text {th }}$ cycle is kept under control too.

Theorem 2 is proven in Appendix A. There, we introduce a novel notion of weight to describe local stochastic errors: the closeness weight. We then present the stochastic shadow decoder, a variant of the (adversarial) shadow decoder of Definition 3. Importantly, on confined codes, it keeps the closeness weight of the residual error under control over repeated correction cycles. Finally, the proof of Theorem 2 follows by combining these results with some classic percolation theory bounds.

The proof of Theorem 3 is very technical and is deferred to Appendix C. It is an adaption of the one of soundness for 4D codes given in Ref. [18], and it is reported in this paper for completeness. We remind the reader that, for our numerical studies on 3D product codes, we do not use the shadow decoder, but rather heuristics that perform well in practice. In particular, we use a two-stage decoder that exploits a metacheck structure on syndromes and attempts to repair the syndrome if and only if it does not pass all metachecks (see Sec. V C).

Our main motivation to introduce the concept of confinement and the shadow decoder was to find a feature of codes able to encompass all known examples of singleshot codes. Campbell [18] introduced the notion of soundness and showed that this property is a sufficient condition for codes to show single-shot properties in the adversarial setting. Nonetheless, Fawzi et al. [22] showed that expander codes have a single-shot threshold for local stochastic noise, even though they do not have the soundness property. As already said though, expander codes do have confinement. In Corollary 9 of Ref. [20] the authors prove that their confinement function is linear and call this property robustness. Confinement, in other words, fills the gap leaved by the concept of soundness. Furthermore, as Lemma 2 states, it is a requirement strictly weaker than soundness: any LDPC family of codes with good soundness has good confinement.

Definition 4 (Soundness [18]). Let $t$ be an integer and $f: \mathbb{Z} \rightarrow \mathbb{R}$ be a function with $f(0)=0$. Given a stabilizer code with syndrome map $\sigma(\cdot)$ we say it is $(t, f)$ sound if for all error sets e with $|\sigma(e)| \leq t$ it follows that $f(|\sigma(e)|) \geq|e|^{\text {red }}$.

Definition 5 (Good soundness [18]). Consider an infinite family of codes with syndrome maps $\sigma_{n}(\cdot)$. We say that the family has good soundness if each code in it is $(t, f)$ sound where the following holds:

1. t grows with $n$ such that $t \in \Omega\left(n^{b}\right)$ with $b>0$;

2. and $f(\cdot)$ is monotonically increasing and independent of $n$.
It follows easily from Campbell's definition of soundness and our definition of confinement that the former entails the latter.

Lemma 2. Consider a LDPC code that is $(t, f)$ sound with $f$ increasing. If its qubit degree is at most $\omega$, then it has $((t / \omega), f)$ confinement.

If $e$ is an error set with $|e|^{\text {red }} \leq t / \omega$, for its syndrome the following holds:

$$
|\sigma(e)| \leq \frac{t}{\omega} \times \omega=t .
$$

By soundness of the code,

$$
f(|\sigma(e)|) \geq|e|^{\text {red }} .
$$

In conclusion, confinement successfully describes general and inclusive properties related to single-shot error correction. More than that, good confinement is a requirement strictly weaker than good soundness as the following example illustrates. We consider the quantum expander code family of Ref. [20], which has the following four properties: (i) they have full-rank parity-check matrices; (ii) they have $(t, 3 x)$ confinement, with $t \in \Omega(d)$; (iii) for every small error $e$ with $|e| \leq 3$, we have $|\sigma(e)|>1$ (see Appendix D for details).

By property (i), every syndrome is a valid syndrome and we can consider some weight-1 syndrome $s$. Assume to the contrary that there exists an $|e| \leq t$ giving the syndrome $s=\sigma(e)$, then by $(t, 3 x)$ confinement

$$
3|\sigma(e)| \geq|e|^{\mathrm{red}},
$$

and plugging in $|\sigma(e)|=1$ gives

$$
3 \geq|e|^{\mathrm{red}} \text {. }
$$

We know by property (iii) that this would entail $|\sigma(e)|>1$, which leads to a contraction. Therefore, the assumption $|e| \leq t$ must be false and so $|e|>t$.

Therefore, if the code family had $(\tau, \varphi)$ soundness, then

$$
\varphi(1)>t \text {. }
$$

Because $\varphi$ is the same across the whole family, and $t \in$ $\Omega(d)$ increases proportionally to the code distance, by considering bigger codes if necessary, this leads to a contradiction. In other words, we show that this family of expander codes with good linear confinement cannot have good soundness.

The remainder of this paper is devoted to the study of the $3 \mathrm{D}$ product codes. We recall their construction in Sec. IV and we numerically assess their single-shot performance under local stochastic noise in Sec. V. 


\section{CODE CONSTRUCTION}

The identification of Pauli operators $p \in \mathcal{P}_{n}$ with binary vectors $\bar{p}=\left(\bar{p}_{X}, \bar{p}_{Z}\right) \in \mathbb{F}_{2}^{2 n}$ is a group homomorphism (i.e., multiplication of Pauli operators corresponds to the sum of their vector representation in $\mathbb{F}_{2}^{2 n}$ ) and because $\sigma(\cdot)$ is linear, syndrome measurement can be simulated via a matrix-vector product:

$$
\begin{aligned}
\sigma: \mathbb{F}_{2}^{2 n} & \longrightarrow \mathbb{F}_{2}^{m} \\
\left(\begin{array}{c}
\bar{p}_{X} \\
\bar{p}_{Z}
\end{array}\right) & \mapsto H\left(\begin{array}{l}
\bar{p}_{X} \\
\bar{p}_{Z}
\end{array}\right),
\end{aligned}
$$

where the vector $\left(\bar{p}_{X}, \bar{p}_{Z}\right)^{T} \in \mathbb{F}_{2}^{2 n}$ represents a Pauli error on the physical qubits. Following the nomenclature from classical coding theory, we refer to the syndrome matrix $H$ as parity-check matrix and we say that a code is LDPC when its parity check is low density.

A stabilizer code is a Calderbank-Shor Steane (CSS) code if its stabilizer group can be generated by the disjoint union of a set of $X$ operators and a set of $Z$ operators. In this case, its parity check is a block matrix:

$$
H=\left(\begin{array}{c|c}
0 & H_{X} \\
\hline H_{Z} & 0
\end{array}\right),
$$

where $H_{X}$ has size $m_{x} \times n$ and $H_{Z}$ has size $m_{z} \times n$ if the generating set of $X$ stabilizers and $Z$ stabilizers has cardinality $m_{x} / m_{z}$. Equation (14) entails that syndrome extraction can be performed separately for the $X$ component and for the $Z$ component. In fact, if a Pauli operator has vector representation $\left(\bar{p}_{X}, \bar{p}_{Z}\right)^{T}=\left(\bar{p}_{X}, 0\right)^{T}+\left(0, \bar{p}_{Z}\right)^{T} \in \mathbb{F}_{2}^{2 n}$, then for its syndrome the following holds:

$$
\begin{aligned}
H\left(\begin{array}{c}
\bar{p}_{X} \\
\bar{p}_{Z}
\end{array}\right) & =H\left(\begin{array}{c}
\bar{p}_{X} \\
0
\end{array}\right)+H\left(\begin{array}{c}
0 \\
\bar{p}_{Z}
\end{array}\right) \\
& =\left(\begin{array}{c}
0 \\
H_{Z} \bar{p}_{X}
\end{array}\right)+\left(\begin{array}{c}
H_{X} \bar{p}_{Z} \\
0
\end{array}\right) \\
& =\left(\begin{array}{c}
0 \\
\bar{s}_{Z}
\end{array}\right)+\left(\begin{array}{c}
\bar{s}_{X} \\
0
\end{array}\right),
\end{aligned}
$$

where $\bar{s}_{Z} \in \mathbb{F}_{2}^{m_{z}}$ and $\bar{s}_{X} \in \mathbb{F}_{2}^{m_{x}}$. In other words, it is possible to truncate these vectors without losing information and deal with $X$ and $Z$ operators separately. For this reason, we say that a CSS code is provided with two syndrome maps, which correspond to the two blocks or matrices $H_{X}$ and $H_{Z}$, respectively. Accordingly, a CSS code will have a $X$ distance and a $Z$ distance and can be compactly refereed to as a $\left[\left[n, k, d_{x}, d_{z}\right]\right]$ code.

For our purposes, it is useful to describe CSS codes in terms of chain complexes. We first explain how a CSS code yields a chain complex and then how to define valid CSS codes starting from chain complexes. This last step ultimately allows us to use a standard method to iteratively build chain complexes, namely the product of complexes, to build interesting CSS codes (see, for instance, Ref. [38] for a comprehensive discussion on the subject).

Consider a CSS code $\mathcal{C}$ defined by the syndrome maps $H_{X}$ and $H_{Z}$ of size $m_{x} \times n$ and $m_{z} \times n$ respectively. The sequence of maps and vector spaces,

$$
\mathbb{F}_{2}^{m_{z}} \stackrel{H_{Z}^{T}}{\longrightarrow} \mathbb{F}_{2}^{n} \stackrel{H_{X}}{\longrightarrow} \mathbb{F}_{2}^{m_{x}}
$$

contains all the information needed to define $\mathcal{C}$. In fact, the dimension of the vector space in the middle, $n$, is the length of the code, and the dimensions $m_{x}$ and $m_{z}$ of the external spaces are, respectively, the number of $X$ and $Z$ stabilizer generators. The logical dimension $k$ of the code $\mathcal{C}$ equates to

$$
\begin{aligned}
k & =\operatorname{dim}\left(\operatorname{ker} H_{X}\right)-\operatorname{dim}\left(\operatorname{Im} H_{Z}^{T}\right) \\
& =\operatorname{dim}\left(\operatorname{ker} H_{Z}\right)-\operatorname{dim}\left(\operatorname{Im} H_{X}^{T}\right) .
\end{aligned}
$$

We use ker $H$ for the kernel of $H$, which is the set of all $v$ such that $H v=0$. We use $\operatorname{Im} H$ for the image of $H$, which is the set of all vectors $w$ that can be written as $w=H v$ for some $v$. The distances $d_{x}$ and $d_{z}$ are given by

$$
\begin{aligned}
& d_{x}=\min \left\{|v| \text { such that } v \in \operatorname{ker} H_{Z}, v \notin \operatorname{Im}\left(H_{X}^{T}\right)\right\}, \\
& d_{z}=\min \left\{|v| \text { such that } v \in \operatorname{ker} H_{X}, v \notin \operatorname{Im}\left(H_{Z}^{T}\right)\right\} .
\end{aligned}
$$

Lastly, because rows of $H_{X}$ and $H_{Z}$ represent the support of $X$ and $Z$ stabilizer generators, respectively, we can also verify that $X$ and $Z$ stabilizers commute by assuring that the scalar product of each row of $H_{X}$ and any row of $H_{Z}$ (or equivalently each row of $H_{Z}$ and any row of $H_{X}$ ) is 0 in $\mathbb{F}_{2}$. In fact, this is equivalent to verifying that the supports of any two $X$ and $Z$ stabilizer generators have even overlap and therefore that they represent commuting Pauli operators. In other words, for $H_{X}$ and $H_{Z}$ it holds that

$$
H_{X} H_{Z}^{T}=0 \in \mathbb{F}_{2}^{m_{x} \times m_{z}} .
$$

To sum up, we completely define the CSS code $\mathcal{C}$ in terms of the sequence described by Eq. (15), which in homology theory is referred to as a length-2 chain complex. As we now detail the converse is also true, and any chain complex of length 2 or greater determines a CSS code.

A length $\ell$ chain complex $\mathfrak{C}$ is a collection of vector spaces $C^{0}, \ldots, C^{\ell}$ and linear maps $\delta_{i}: C^{i} \rightarrow C^{i+1}$ :

$$
C^{0} \stackrel{\delta_{0}}{\rightarrow} C^{1} \rightarrow \cdots \rightarrow C^{i} \stackrel{\delta_{i}}{\rightarrow} C^{i+1} \rightarrow \cdots \rightarrow C^{\ell}
$$

with the only constraint

$$
\delta_{i} \delta_{i-1}=0,
$$

for $i=1, \ldots, \ell-1$. Whenever the spaces $C^{i}$ are vector spaces on the binary field $\mathbb{F}_{2}$ and $\ell \geq 2$, we can define a 
CSS code on $\mathfrak{C}$. To see how this is the case, let $0<i \leq$ $\ell-1$. We define a CSS code $\mathcal{C}\left(\mathfrak{C}_{i}\right)$ on the chain complex $\mathfrak{C}$ by equating

$$
H_{Z}=\delta_{i-1}^{T}, H_{X}=\delta_{i}
$$

Notice that the defining property of chain complexes, Eq. (17), entails that our choice, Eq. (18), for $H_{X}$ and $H_{Z}$ is valid: the stabilizer group generated by the $X$ and $Z$ operators supported on rows of $H_{X}$ and $H_{Z}$ respectively, is Abelian. Therefore, the unique CSS code $\mathcal{C}\left(\mathfrak{C}_{i}\right)$ associated to the syndrome maps given in Eq. (18) is well defined. Importantly, the parameters $\left[\left[n, k, d_{x}, d_{z}\right]\right]$ of $\mathcal{C}\left(\mathfrak{C}_{i}\right)$ all have a translation in the chain-complex language. Using such terminology, we say that the code has length $n=\operatorname{dim}\left(C_{i}\right)$. It is known that the number of logical qubits $k$ is equal to the dimension of the $i$ th homology group $\mathcal{H}_{i}$ or, equivalently, the dimension of the $(i-1)$ th cohomology group $\mathcal{H}_{i+1}^{*}$, defined, respectively, as the quotient groups:

$$
\begin{aligned}
\mathcal{H}_{i} & =\operatorname{ker} \delta_{i} / \operatorname{Im} \delta_{i-1}, \\
\mathcal{H}_{i+1}^{*} & =\operatorname{ker} \delta_{i-1}^{T} / \operatorname{Im} \delta_{i}^{T} .
\end{aligned}
$$

The $X$ distance equates the minimum weight of any nonzero vector in $\mathcal{H}_{i-1}^{*}$, while the $Z$ distance is the minimum weight of any nonzero vector in $\mathcal{H}_{i}$. It is easy to verify that these definitions in terms of homology and cohomology are actually equivalent to the ones given above for the CSS code described by Eq. (15); we refer the interested reader to Ref. [39] for a detailed presentation of homology theory.

We introduce the homology language because it allows us to succinctly describe the class of $3 \mathrm{D}$ product codes studied here. By 3D product codes we refer to the CSS codes derived by the homological product of three length1 chain complexes, as described in Ref. [40]. Given three classical linear codes with parity-check matrices $\delta_{A}, \delta_{B}$, and $\delta_{C}$ they define three length- 1 chain complexes:

$$
\begin{aligned}
& \delta_{A}: C_{A}^{0} \longrightarrow C_{A}^{1}, \\
& \delta_{B}: C_{B}^{0} \longrightarrow C_{B}^{1}, \\
& \delta_{C}: C_{C}^{0} \longrightarrow C_{C}^{1},
\end{aligned}
$$

where $C_{\ell}^{0}=\mathbb{F}_{2}^{n_{\ell}}$ and $C_{\ell}^{1}=\mathbb{F}_{2}^{m_{\ell}}$ if $\delta_{\ell}$ has size $m_{\ell} \times n_{\ell}$ for $\ell=A, B, C$. By using tensor product and direct sum of vector spaces and maps, we can combine these three chain complexes to build a bigger length-3 chain complex.

The tensor product is denoted by the symbol $\otimes$. Given two vector spaces $A$ and $B$ over a field $\mathbb{F}$, their tensor product is the vector space $A \otimes B$ generated by the formal sums $\sum a \otimes b$ where $a \in A$ and $b \in B$ and the operator $\otimes$ is bilinear, i.e., for any $a_{1}, a_{2}, b_{1}, b_{2}$ in $A$ and $B$, respectively, it holds that

$$
\begin{aligned}
& \left(a_{1}+a_{2}\right) \otimes b_{1}=a_{1} \otimes b_{1}+a_{2} \otimes b_{1}, \\
& a_{1} \otimes\left(b_{1}+b_{2}\right)=a_{1} \otimes b_{1}+a_{1} \otimes b_{2} .
\end{aligned}
$$

If $\alpha: A \rightarrow A^{\prime}$ and $\beta: B \rightarrow B^{\prime}$ are linear maps, their tensor product is defined as the linear map:

$$
\begin{aligned}
\alpha \otimes \beta: A \otimes B & \longrightarrow A^{\prime} \otimes B^{\prime}: \\
a \otimes b & \longmapsto \alpha(a) \otimes \beta(b) .
\end{aligned}
$$

It is of course possible to iterate this construction and define the tensor product of three (or more) spaces and maps, as we do now in order to obtain a length-3 chain complex $\mathfrak{C}^{\prime \prime \prime}$ from the seed matrices $\delta_{A}, \delta_{B}, \delta_{C}$. The chain complex $\mathfrak{C}^{\prime \prime \prime}$ is compactly described by the sequence of spaces and maps:

$$
\mathcal{C}_{0} \stackrel{\delta_{0}}{\rightarrow} \mathcal{C}_{1} \stackrel{\delta_{1}}{\rightarrow} \mathcal{C}_{2} \stackrel{\delta_{2}}{\rightarrow} \mathcal{C}_{3}
$$

which correspond to the tensor-product structure:
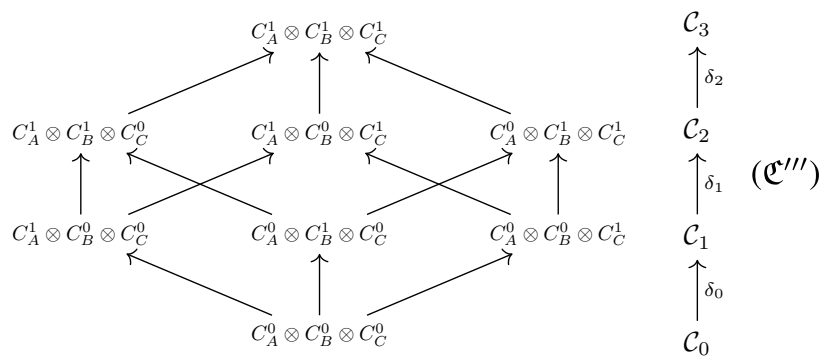

where

$$
\begin{aligned}
& \mathcal{C}_{0}=C_{A}^{0} \otimes C_{B}^{0} \otimes C_{C}^{0}, \\
& \mathcal{C}_{1}=C_{A}^{1} \otimes C_{B}^{0} \otimes C_{C}^{0} \oplus C_{A}^{0} \otimes C_{B}^{1} \otimes C_{C}^{0} \oplus C_{A}^{0} \otimes C_{B}^{0} \otimes C_{C}^{1}, \\
& \mathcal{C}_{2}=C_{A}^{1} \otimes C_{B}^{1} \otimes C_{C}^{0} \oplus C_{A}^{1} \otimes C_{B}^{0} \otimes C_{C}^{1} \oplus C_{A}^{0} \otimes C_{B}^{1} \otimes C_{C}^{1}, \\
& \mathcal{C}_{3}=C_{A}^{1} \otimes C_{B}^{1} \otimes C_{C}^{1},
\end{aligned}
$$

and

$$
\delta_{0}=\left(\begin{array}{c}
\delta_{A} \otimes \mathbb{1} \otimes \mathbb{1} \\
\mathbb{1} \otimes \delta_{B} \otimes \mathbb{1} \\
\mathbb{1} \otimes \mathbb{1} \otimes \delta_{C}
\end{array}\right),
$$




$$
\begin{aligned}
& \delta_{1}=\left(\begin{array}{ccc}
\mathbb{1} \otimes \delta_{B} \otimes \mathbb{1} & \delta_{A} \otimes \mathbb{1} \otimes \mathbb{1} & 0 \\
\mathbb{1} \otimes \mathbb{1} \otimes \delta_{C} & 0 & \delta_{A} \otimes \mathbb{1} \otimes \mathbb{1} \\
0 & \mathbb{1} \otimes \mathbb{1} \otimes \delta_{C} & \mathbb{1} \otimes \delta_{B} \otimes \mathbb{1}
\end{array}\right), \\
& \delta_{2}=\left(\mathbb{1} \otimes \mathbb{1} \otimes \delta_{C} \quad \mathbb{1} \otimes \delta_{B} \otimes \mathbb{1} \quad \delta_{A} \otimes \mathbb{1} \otimes \mathbb{1}\right) .
\end{aligned}
$$

It is easy to verify that the chain complex $\left(\mathfrak{C}^{\prime \prime \prime}\right)$ satisfies condition (17) for $i=1, \ldots, 2$ and it is therefore well defined. As done above, we define a $\operatorname{CSS} \operatorname{code} \mathcal{C}\left(\delta_{A}, \delta_{B}, \delta_{C}\right)$ on $\left(\mathfrak{C}^{\prime \prime \prime}\right)$ by equating

$$
H_{Z}=\delta_{0}^{T}, H_{X}=\delta_{1} .
$$

We refer to the matrix $M=\delta_{2}$ as the metacheck matrix for the $X$ stabilizers. Condition (18) entails $M H_{X}=0$ and as a consequence we can think of the matrix $M$ as a paritycheck matrix on the $X$ syndromes: any valid $X$ syndrome satisfies the constraints defined by $M$.

Remarkably, the parameters of the code $\mathcal{C}\left(\delta_{A}, \delta_{B}, \delta_{C}\right)$ can be derived in terms of the properties of the seed matrices $\delta_{A}, \delta_{B}, \delta_{C}$. In fact, let $\left[n_{\ell}, k_{\ell}, \delta_{\ell}\right] /\left[n_{\ell}^{T}, k_{\ell}^{T}, d_{\ell}^{T}\right]$ be the parameters of the classical linear code with parity-check matrix $\delta_{\ell} / \delta_{\ell}^{T}, \ell=A, B, C$. As shown in Ref. [40], the chain complex $\left(\mathfrak{C}^{\prime \prime \prime}\right)$ yields an $\left[\left[n, k, d_{x}, d_{z}\right]\right]$ code such that, if $k \neq 0$,

$$
\begin{aligned}
n & =n_{a}^{T} n_{b} n_{c}+n_{a} n_{b}^{T} n_{c}+n_{a} n_{b} n_{c}^{T}, \\
k & =k_{a}^{T} k_{b} k_{c}+k_{a} k_{b}^{T} k_{c}+k_{a} k_{b} k_{c}^{T}, \\
d_{x} & =\min \left\{d_{a}^{T}, d_{b}^{T}, d_{c}^{T}\right\}, \\
d_{z} & =\min \left\{d_{b} d_{c}, d_{a} d_{c}, d_{a} d_{b}\right\} .
\end{aligned}
$$

By convention, the distance of a code with dimension 0 is $\infty$. We define the single-shot distance $d_{\mathrm{SS}}[18]$ of the chain complex $\left(\mathfrak{C}^{\prime \prime \prime}\right)$ as the minimum weight of a vector in $\mathcal{C}_{2}$ that satisfies all the constraints given by $\delta_{2}$ (i.e., it belongs to the kernel of $\delta_{2}$ ) but is not a valid $X$ syndrome (i.e., it does not belong to the image of $\delta_{1}$ ). In other words, $d_{\mathrm{SS}}$ is the minimum weight of a vector in the second homology group $\mathcal{H}_{2}=\operatorname{ker} \delta_{2} / \operatorname{Im} \delta_{1}$ of the chain complex $\mathcal{C}$. Following Ref. [40] it is easy to verify that $d_{\mathrm{SS}}=\min \left\{d_{a}, d_{b}, d_{c}\right\}$ if $\mathcal{H}_{2} \neq 0$ and $\infty$ otherwise.

It is important to note that, if the matrices $\delta_{\ell}$ are LDPC, then their 3D product code is quantum LDPC. In fact, if $\delta_{\ell}$ has column (row) of weight bounded by $c_{\ell}\left(r_{\ell}\right)$, then $\delta_{i}$ has column and row weight bounded by $c_{i}$ and $r_{i}$, respectively, where

\footnotetext{
i. $c_{0} \leq c_{a}+c_{b}+c_{c}$ and $r_{0} \leq \max \left\{r_{a}, r_{b}, r_{c}\right\}$;

ii. $c_{1} \leq \max \left\{c_{a}+c_{b}, c_{a}+c_{c}, c_{b}+c_{c}\right\}$

and

$r_{1} \leq \max \left\{r_{a}+r_{b}, r_{a}+r_{c}, r_{b}+r_{c}\right\}$

iii. $c_{2} \leq \max \left\{c_{a}, c_{b}, c_{c}\right\}$ and $r_{2} \leq r_{a}+r_{b}+r_{c}$.
}

\section{A. On geometric locality}

In addition to preserving the LDPC properties of the seed matrices, the 3D product yields local codes when qubits are placed on edges of a 3D cubic lattice. We defer the reader to Appendix B for a thorough discussion on the embedding of $3 \mathrm{D}$ product codes on a cubic lattice and we here present a loose summary.

Qubits of a 3D product code associated to the chain complex $\left(\mathfrak{C}^{\prime \prime \prime}\right)$ are in bijection with basis elements of the space $\mathcal{C}_{1}$; since $\mathcal{C}_{1}$ is the direct sum of the three vector spaces $C_{A}^{1} \otimes C_{B}^{0} \otimes C_{C}^{0}, C_{A}^{0} \otimes C_{B}^{1} \otimes C_{C}^{0}$ and $C_{A}^{0} \otimes C_{B}^{0} \otimes C_{C}^{1}$ we introduce three different types of qubits: transverse, vertical, and horizontal. Qubit types naturally correspond to the three different orientations of edges on a cubic lattice, namely edges parallel to each of the three crystal planes. Referring to this particular display of qubits, the stabilizers of the code defined by Eq. $\left(\mathfrak{C}^{\prime \prime \prime}\right)$ have support as follows:

1. $X$ stabilizers have support on a two-dimensional (2D) cross of qubits of two types out of three, contained in one of the three crystal planes; the crossing is defined by a square face of a cube (see Fig. 7);

2. $Z$ stabilizers have support on a 3D cross of qubits, with crossing defined by a vertex of a cube (see Fig. 8).

The cubic lattice considered can present some irregularities: in general it is a cubic lattice with some missing edges. Nonetheless, square faces and vertices are uniquely defined and they correspond to a stabilizer every time they contain at least one edge. More specifically, a square face identifies two perpendicular lines of edges i.e., qubits on a plane, which are the edges parallel to the boundary of the square face itself. The corresponding $X$ stabilizer has support contained on those lines of edges i.e., qubits. Similarly, a vertex identifies three perpendicular lines of qubits and the corresponding $Z$ stabilizer has support there contained. When combined with some locality properties of the seed matrices, this characteristic "cross shape" of the stabilizers support entails that 3D product codes are local on a cubic lattice (Proposition 1 in Appendix B). Here, by locality, we mean that for some positive integer $\rho$, the following hold:

1. any $X$-stabilizer generator has weight at most $2 \rho$ with support contained in a 2D box of size $\rho \times \rho$;

2. any $Z$-stabilizer generator has weight at most $3 \rho$ with support contained in a 3D box of size $\rho \times \rho \times$ $\rho$.

Interestingly, it follows easily as a corollary of our locality proof that the 3D toric and surface codes are in fact 3D product codes. We now detail an explicit construction of the $3 \mathrm{D}$ toric and surface codes as $3 \mathrm{D}$ product codes and we refer the reader to Appendix B for further details. 
The $3 \mathrm{D}$ toric code is the $3 \mathrm{D}$ product code obtained by choosing $\delta_{A}=\delta_{B}=\delta_{C}=\delta$ as seed matrices, where $\delta$ is the parity-check matrix of the repetition code. For instance, the $3 \mathrm{D}$ toric code with linear lattice size $L=3$ is given by

$$
\delta=\left(\begin{array}{lll}
1 & 1 & 0 \\
0 & 1 & 1 \\
1 & 0 & 1
\end{array}\right)
$$

In general, the 3D toric code of lattice size $L$, has parameters

$$
\left[\left[3 L^{3}, 3, L, L^{2}\right]\right]
$$

and single-shot distance $d_{\mathrm{SS}}=L$.

The 3D surface code is obtained from this construction by choosing, for linear lattice size $L=3$,

$$
\delta_{A}=\delta_{B}=\left(\begin{array}{lll}
1 & 1 & 0 \\
0 & 1 & 1
\end{array}\right)
$$

and

$$
\delta_{C}=\left(\begin{array}{lll}
1 & 1 & 0 \\
0 & 1 & 1
\end{array}\right)^{T}
$$

Therefore, for lattice size $L$, it has parameters

$$
\left[\left[2 L(L-1)^{2}+L^{3}, 1, L, L^{2}\right]\right]
$$

and single-shot distance $d_{\mathrm{SS}}=\infty$. Further details can be found in Appendix B.

It should be noted that the surface and toric codes are special instances of 3D product codes that have geometrically local stabilizers. This is beneficial, as it means they could be implemented on a quantum computer using only nearest-neighbor (in 3D) interactions between qubits. The disadvantage of the $3 \mathrm{D}$ surface and toric codes is that they have fixed dimension, encoding only one and three qubits, respectively. The $3 \mathrm{D}$ product construction can be used to obtain codes that are not geometrically local, but have improved encoding rates over the surface and toric codes. We refer to these codes as "nontopological" codes and investigate their decoding in Sec. VE.

\section{NUMERICS}

To assess the single-shot performance of the $3 \mathrm{D}$ product codes, we simulate the decoding of phase-flip $(Z)$ errors. As 3D product codes are CSS codes, the relevant stabilizers are the $X$ stabilizers. Let $\bar{e}_{Z} \in \mathbb{F}_{2}^{n}$ describe the support of a phase-flip error, i.e., $\left(\bar{e}_{Z}\right)_{i}=1$ if qubit $i$ has a phase-flip error. The syndrome, $\bar{s}_{X}$, of this error is then

$$
\bar{s}_{X}=H_{X} \bar{e}_{Z},
$$

where $H_{X} \in \mathbb{F}_{2}^{m \times n}$ is the parity-check matrix of the $X$ stabilizers of the code [see Eq. (14)].
Owing to the chain-complex structure of 3D product codes (outlined in Sec. IV) the syndromes $\bar{s}_{X}$ are themselves the codewords of a classical linear code with paritycheck matrix $M$ such that $M \bar{s}_{X}=0$ for all $\bar{s}_{X} \in \operatorname{Im}\left(H_{X}\right)$. We refer to such a code on the syndromes as a metacode. The metacheck matrix can be used to detect and correct syndrome noise.

In a two-stage single-shot decoder, stage-1 decoding corrects the syndrome noise using $M$ before stage- 2 decoding corrects the data qubits. In general, decoding is an NP-complete problem that cannot be solved exactly in polynomial time. However, good heuristic techniques exist that allow approximate solutions to be efficiently computed. In this work, we use two such decoding methods: minimum-weight perfect matching and belief propagation plus ordered statistics decoding. Both MWPM and $\mathrm{BP}+\mathrm{OSD}$ are algorithms that run over graphical models that encapsulate the structure of the code. We now briefly describe each decoder.

\section{A. Minimum-weight perfect matching}

The minimum-weight perfect-matching decoder is useful for codes in which chains of errors produce weight-2 syndromes. The method works by mapping the decoding problem to a graph where nodes represent the code's stabilizer generators and weighted edges represent error chains of different lengths. For a given pair of unsatisfied stabilizers, MWPM deduces the shortest error chain that could have caused it [41].

MWPM finds use for a variety of topological codes, most famously for the 2D surface and toric codes [2,4245]. For $3 \mathrm{D}$ codes, MWPM is a suitable candidate for the syndrome-repair step referred to as stage-1 decoding. Specifically, the syndrome of a phase-flip error can be viewed as a collection of closed loops of edges in a simple cubic lattice [46] (with boundary conditions depending on the code). Measurement errors cause loops of syndrome to be broken, and the job of stage- 1 decoding is to repair these broken syndromes. To obtain the corresponding matching problem, we create a complete graph whose vertices correspond to the break-points of the broken syndrome loops, with edge weights that are equal to the path lengths between the break points. We use the Blossom V [47] implementation of Edmonds's algorithm to solve this matching problem. The edges in the matching correspond to the syndrome-recovery operators.

\section{B. Belief propagation + ordered statistics decoding}

Belief propagation is an algorithm for performing inference on sparse graphs that finds widespread use in highperformance classical coding. Classical LDPC codes, for example, achieve performance close to the Shannon limit when decoded with BP [48]. In the context of quantum 
coding, BP is useful for codes that do not produce pairs of syndromes and therefore cannot be decoded with MWPM.

The BP algorithm maps the decoding problem to a bipartite factor graph where the two node species represent data qubits and syndromes, respectively. Graph edges are drawn between the data and syndrome nodes according to the code's parity-check matrix. The factor graph is designed to provide a factorization of the probability distribution that describes the relationship between syndromes and errors. The BP algorithm proceeds by iteratively passing "beliefs" between data and syndrome nodes, at each step updating the probability that a data node is errored. The algorithm terminates once the probability distribution implies an error pattern that satisfies the inputted syndrome. For a full description of the BP algorithm we direct the reader to Ref. [49].

For quantum codes, the standard BP algorithm alone does not achieve good decoding performance due to the presence of degenerate errors. These cause "split beliefs" and prevent the algorithm from terminating. Various methods have been proposed for adapting BP decoding to quantum codes [27,50-54]. A particularly effective recent proposal involves combining BP with a postprocessing technique known as ordered statistics decoding [55]. The OSD step uses the probability distribution outputted by BP to select a low-weight recovery operator that satisfies the syndrome equation.

The BP+OSD algorithm was first applied to quantum expander codes by Panteleev and Kalachev [55]. Following this, Roffe et al. [56] demonstrated that the BP+OSD decoder applies more widely across a broad range of quantum-LDPC codes, including the 2D surface and toric codes. For this work, we use the software implementation of BP+OSD from Ref. [56], which can be downloaded from Ref. [57].

\section{The two-stage single-shot decoding algorithm}

Our simulations of the two-stage single-shot decoder employ two strategies. (1) MWPM and BP+OSD: stage-1 decoding is performed using MWPM and stage- 2 decoding uses $\mathrm{BP}+\mathrm{OSD}$. (2) $\mathrm{BP}+\mathrm{OSD} \times 2$ : both stages are $\mathrm{BP}+\mathrm{OSD}$.

Algorithm 1 describes our methodology for the simulations of the two-stage single-shot decoder.

The 3D toric code has a failure mode that is not present in the 3D surface code. In such codes, syndromes $\bar{s}_{X}$ exist that satisfy all of the metachecks, $M \bar{s}_{X}=0$, but are invalid syndromes, meaning that $\bar{s}_{X}$ does not belong to the image of $H_{X}$. In other words, $\bar{s}_{X}$ is invalid if there is no error vector $\bar{e}_{Z} \in \mathcal{C}_{1}$ with syndrome $\bar{s}_{X}$ but $\bar{s}_{X}$ is a codeword of the metacode.

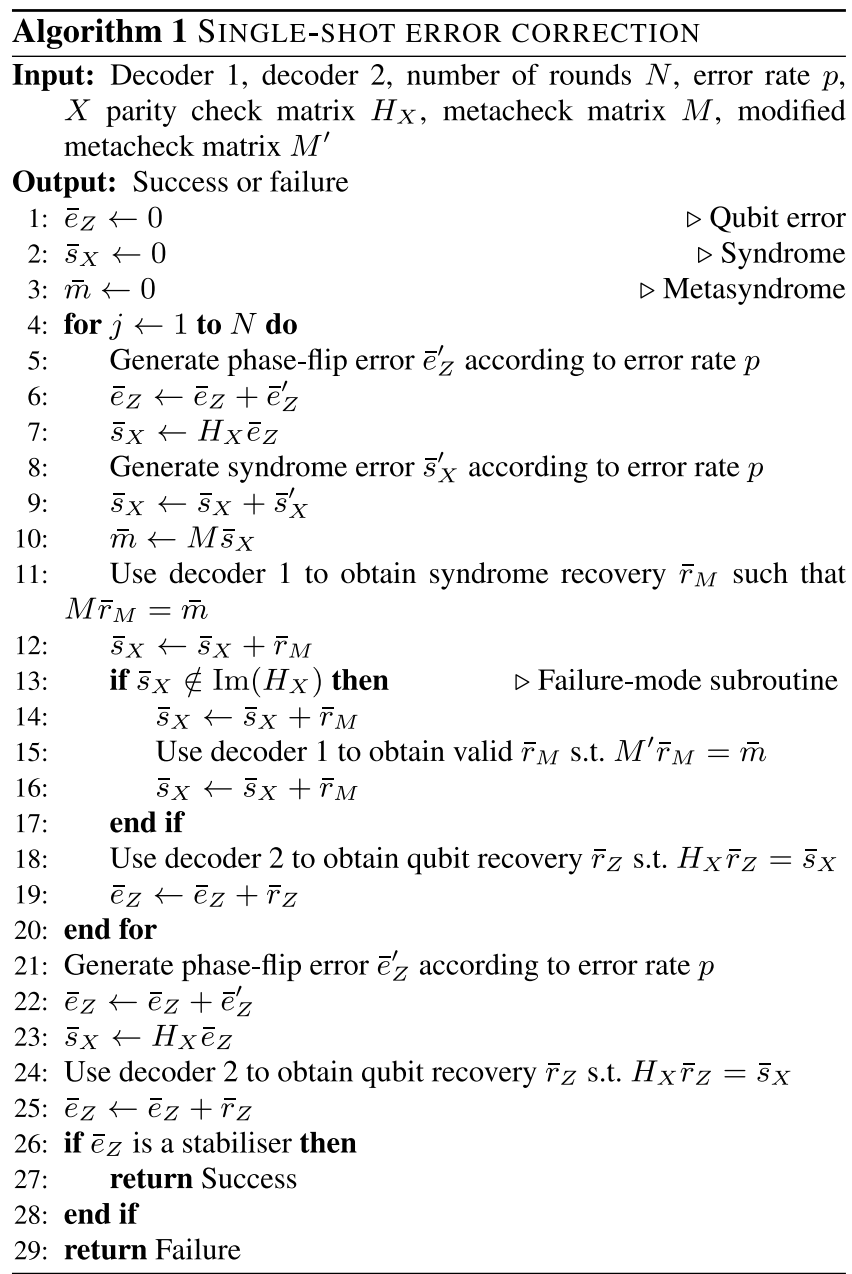

More generally, referring to the chain complex $\left(\mathfrak{C}^{\prime \prime \prime}\right)$ :

$$
\mathcal{C}_{0} \underset{H_{Z}^{T}}{\stackrel{\delta_{0}}{\longrightarrow}} \mathcal{C}_{1} \underset{H_{X}}{\stackrel{\delta_{1}}{\longrightarrow}} \mathcal{C}_{2} \underset{M}{\stackrel{\delta_{2}}{\longrightarrow}} \mathcal{C}_{3}
$$

we see that nonvalid syndromes do exist whenever $\operatorname{Im} \delta_{1} \subsetneq$ $\operatorname{ker} \delta_{2}$. In the homology language, we say that invalid syndromes are nontrivial elements of the second homology group:

$$
\mathcal{H}_{2}=\operatorname{ker} \delta_{2} / \operatorname{Im} \delta_{1}=\operatorname{ker} M / \operatorname{Im} H_{X}
$$

If $k_{m}$ is the dimension of $\mathcal{H}_{2}$, the set of invalid syndromes is a vector subspace of $\mathcal{C}_{2}$ of dimension $k_{m}$ whose vectors can be written as $\bar{u}+H_{X} \bar{e}_{Z}$ where $\bar{u}$ is a representative of the equivalence class $[\bar{u}] \in \mathcal{H}_{2}$ and $\bar{e}_{Z} \in \mathcal{C}_{1}$. Thus, if $F_{M}$ is a matrix whose columns are $k_{m}$ vectors in $\mathcal{C}_{2}$ that generate $\mathcal{H}_{2}$ (meaning that they belong to $k_{m}$ different equivalence classes in $\mathcal{H}_{2}$ ), we can write any invalid syndrome $\bar{s}_{X}$ as

$$
\bar{s}_{X}=F_{M} \bar{v}+H_{X} \bar{e}_{Z},
$$


where $\bar{v} \in \mathbb{F}_{2}^{k_{m}}$ is nonzero if and only if $\bar{s}_{X}$ is invalid and $\bar{e}_{Z}$ is any error vector in $\mathcal{C}_{1}$.

By duality on $\left(\mathfrak{C}^{\prime \prime \prime}\right)$, the second cohomolgy group:

$$
\mathcal{H}_{2}^{*}=\operatorname{ker} \delta_{1}^{T} / \operatorname{Im} \delta_{2}^{T}=\operatorname{ker} H_{X}^{T} / \operatorname{Im} M^{T},
$$

has order $k_{m}$ too. If $L_{M}$ is a matrix whose $k_{m}$ rows generates $\mathcal{H}_{2}^{*}$, then the product $\Pi=L_{M} F_{M}$ has full rank $k_{m}$ because both $L_{M}$ and $F_{M}$ have full rank. Moreover, since the rows of $L_{M}$ in particular belongs to $\operatorname{ker} H_{X}^{T}$, it holds $L_{M} H_{X}=0$. Combining these two observations with Eq. (20) yields

$$
\begin{aligned}
L_{M} \bar{s}_{X} & =L_{M} F_{M} \bar{v}+L_{M} H_{X} \bar{e}_{Z} \\
& =\Pi \bar{v},
\end{aligned}
$$

where $\Pi \bar{v}=0$ if and only if $\bar{v}=0$ because $\Pi$ is full rank. In conclusion, we find that

$$
L_{M} \bar{s}_{X} \neq 0
$$

if and only if $\bar{s}_{X}$ is an invalid syndrome. As a consequence, we can assess whether a syndrome is invalid or not by calculating this product. The meaning of matrices $L_{M}$ and $F_{M}$ can be understood by looking at elements in $\mathcal{H}_{2}$ and $\mathcal{H}_{2}^{*}$ as logical operators of a CSS code defined on $\left(\mathfrak{C}^{\prime \prime \prime}\right)$ with qubits in $\mathcal{C}_{2}$ (see Sec. IV). In this setting, the full-rank condition $\operatorname{rank}\left(L_{M} F_{M}\right)=k_{m}$ translates in the anticommuting relation between logical $X$ and logical $Z$ operators of the code.

In the 3D toric code, these invalid syndromes are loops of edges around one of the handles of the torus, and are equivalent to the logical operators of the metacode. It is therefore possible to check whether stage-1 decoding results in such a failure by checking whether the repaired syndrome anticommutes with a matrix $L_{M}$ whose rows generate the relevant group of the logical operators of the metacode. When a metacode failure is encountered, a failure-mode subroutine (line 13 of Algorithm 1) is called that forces the repaired syndrome into the correct form. This subroutine involves using BP+OSD to decode a modified version of the metacheck matrix $M^{\prime}$ defined as follows:

$$
M^{\prime}=\left(\begin{array}{c}
M \\
L_{M}
\end{array}\right)
$$

The additional constraints in the modified metacheck matrix ensure that the repaired syndrome is never an invalid syndrome. We use this subroutine only when we have an invalid syndrome (rather than all the time) as the $L_{M}$ component causes $M^{\prime}$ to have higher maximum row and column weights than $M$, resulting in a reduction in BP decoding performance. Indeed, the rows of $L_{M}$ must have weight lower bounded by the transpose distances of the seed codes [58]. Since the transpose distances of the seed

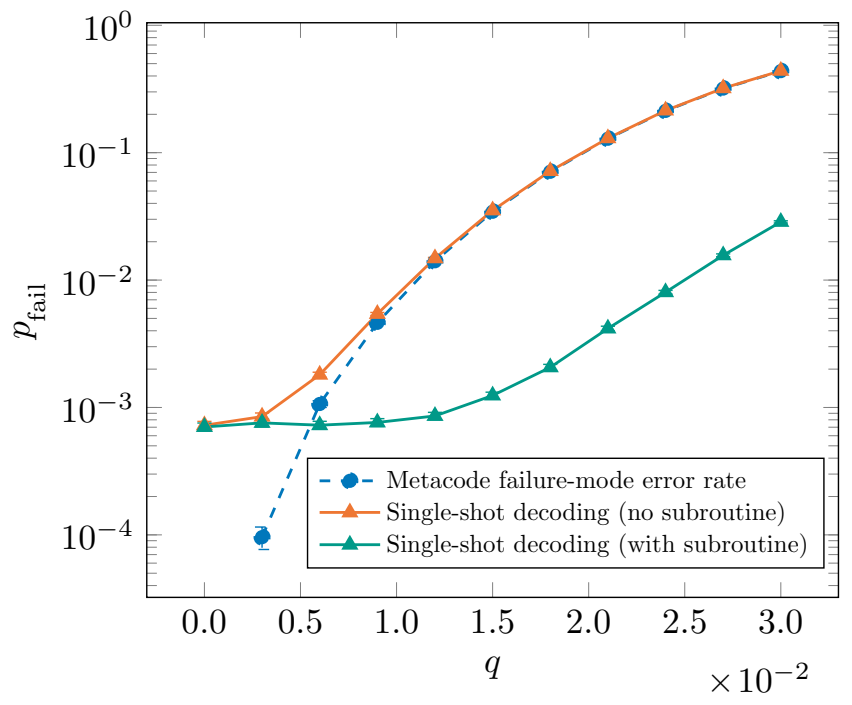

FIG. 2. Single-shot decoding of the 3D toric code with $L=5$, with and without the metacode failure-mode subroutine. The failure rate $p_{\text {fail }}$ is plotted against increasing values of the syndrome error rate $q$, whilst the phase-flip error rate is set to $p=0.1$. Without the subroutine, the single-shot decoder rapidly converges to the failure-mode error rate (dotted line). For large values of $q$ the subroutine improves the logical failure rate by over an order of magnitude. In this simulation, BP+OSD is used for both stage-1 and stage- 2 decoding.

codes also determine the $Z$ distance of the quantum code (Sec. IV), we want these quantities to be growing with the length $n$ of the code and therefore the matrix $L_{M}$ is not, in general, LDPC.

We find that whilst the failure-mode subroutine does not change the error threshold of the decoder, it does considerably reduce the logical error rate for all values of the lattice parameter $L$. This is illustrated for $L=5$ in Fig. 2, which shows the single-shot logical error rate with and without the failure-mode subroutine. For large syndrome error rates, Fig. 2 shows the failure-mode subroutine improves decoding performance by over an order of magnitude.

\section{3D toric and surface codes}

We estimate the sustainable threshold of the 3D toric and surface codes using our two decoding strategies. For codecapacity noise (i.e., perfect syndrome measurements), the syndrome-repair step is not required, so both decoding strategies are the same. For each code family, we observe a code capacity threshold of $p_{\text {th }} \approx 21.6 \%$, as illustrated in Fig. 3. To obtain our threshold estimates, we use the standard critical exponent method [59] (see Appendix E for details). In the single-shot setting, we find similar performance for both our decoding strategies, as summarized in Table II. Our results compare favorably with the performance of other decoders, which we list in Table I. We 


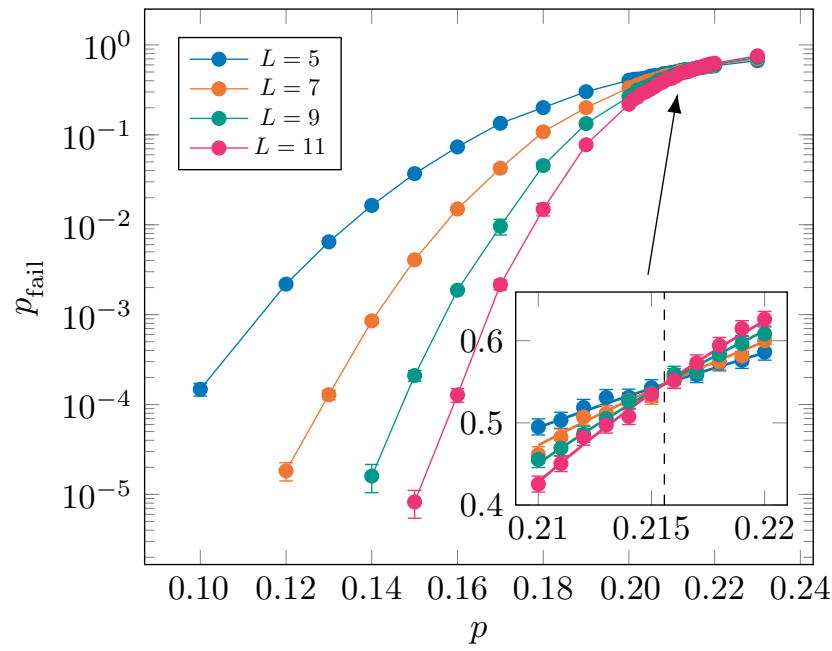

FIG. 3. Code-capacity threshold of the 3D toric code. We plot the logical error rate $p_{\text {fail }}$ as a function of the phase-flip error rate $p$ for codes with linear lattice size $L$. The inset shows an enlargement of the threshold region, where the lines show the threshold fit described in Appendix E. All data points have at least 25 failure events. The error bars show the $95 \%$ confidence intervals $p_{\text {fail }}=\hat{p}_{\text {fail }} \pm 1.96 \sqrt{p_{\text {fail }}\left(1-p_{\text {fail }}\right) / \eta}$, where $\eta \geq 10^{4}$ is the number of Monte Carlo trials.

obtain the highest reported code-capacity threshold and the highest reported single-shot threshold.

We remark that the sustainable threshold that we observe for the 3D toric code is very close to the threshold of MWPM for stringlike errors in the 3D toric code [60]. This implies that the performance of decoder 1 (the syndromerepair step) is limiting the performance of the entire decoding procedure, as was suggested in Ref. [13]. Although the sustainable thresholds we observe for 3D surface codes are slightly higher than for 3D toric codes, the codes we consider are relatively small, which means that boundary effects may be having an impact on our sustainable threshold estimates.

We also investigate the suppression of the logical error rate below threshold in the 3D toric code, using MWPM and $\mathrm{BP}+\mathrm{OSD}$. We use the following ansatz for the logical error rate for values of $p<p_{\text {th }}$,

$$
p_{\text {fail }}(L) \propto\left(p / p_{\text {th }}\right)^{\alpha L^{\beta}},
$$

where $\alpha$ and $\beta$ are parameters to be determined. The code distance of the $3 \mathrm{D}$ toric code for $Z$ errors is $L^{2}$, so if the decoder is correcting errors up to this size, we would expect $\beta \approx 2$. Using the fitting procedure described in Appendix E, we estimate $\beta=1.91$ (3) for $N=0$ (code capacity) and $\beta=1.15$ (3) for $N=8$ (eight rounds of single-shot error correction). Therefore, for the (relatively small) codes that we consider, we find evidence that $\mathrm{BP}+\mathrm{OSD}$ is correcting errors of weight up to the code distance. Viewed as an error-correction problem, the distance of the syndrome-repair step of decoding (i.e., the single-shot distance $d_{\mathrm{SS}}$ ) is $L$, which is consistent with our observed value of $\beta$ in the single-shot case. This provides further evidence that the bottleneck of our single-shot decoding procedure is the syndrome-repair step.

\section{E. Nontopological codes}

Up to this point, we explore the single-shot decoding performance of the $3 \mathrm{D}$ surface and toric codes. As explained in Sec. IV A, the 3D surface and toric codes are topological codes obtained by taking the $3 \mathrm{D}$ product of classical repetition codes. In this section, we extend our numerical analysis to nontopological codes constructed via the $3 \mathrm{D}$ product of random classical codes. Our motivation for investigating nontopological codes is twofold. First, by demonstrating that a random $3 \mathrm{D}$ product code has a sustainable threshold, we provide evidence for our conjecture that the results of Theorems 1 and 3 extend to the stochastic noise setting. Second, we provide evidence that $\mathrm{BP}+\mathrm{OSD}$ is a general decoding method that applies beyond the class of well-studied topological 3D product codes.

Table III shows a family of nontopological codes $\mathcal{C}\left(\delta_{A}, \delta_{B}, \delta_{C}\right)$ obtained by taking the $3 \mathrm{D}$ product of a random code, $\delta_{A}$, with two codes obtained from full-rank repetition codes, $\delta_{B}$ and $\delta_{C}$. For this example, we choose $\delta_{A}$ to be a classical LDPC code constructed by randomly generating parity checks under the constraint that the parity-check matrix $\delta_{A}$ has column and row weights upper bounded by three and four, respectively. The specific advantage of nontopological codes is that they can have nonfixed dimension. For example, the $d=6$ instance of $\mathcal{C}\left(\delta_{A}, \delta_{B}, \delta_{C}\right)$ encodes four logical qubits, whilst the $d=10$ instance encodes ten logical qubits. In contrast, the dimension of the surface and toric codes are fixed at one and three for all code distances. The trade-off is that nontopological codes have nonlocal stabilizer checks, meaning they would have to be implemented on hardware with the ability to perform beyond-nearest-neighbor interactions between

TABLE III. A family of 3D product codes. The seed codes $\left\{\delta_{A}, \delta_{B}, \delta_{C}\right\}$ are set as follows: $\delta_{A}$ is a parity-check matrix of an $[n, k, d]$ LDPC code constructed under the constraint that the column and row weights of its parity-check matrix are upper bounded by three and four, respectively; $\delta_{B}$ is a $[L, 1, L]$ full-rank repetition code; $\delta_{C}$ is the transpose of a $[L, 1, L]$ full-rank repetition code. We denote by $\mathcal{C}\left(\delta_{A}, \delta_{B}, \delta_{C}\right)$ the $3 \mathrm{D}$ product code with seed matrices $\delta_{A}, \delta_{B}, \delta_{C}$. The code distance is set to $\infty$ for codes of dimension 0 .

\begin{tabular}{lccc}
\hline \hline$\delta_{A}$ & $\delta_{B}$ & $\delta_{C}$ & $\mathcal{C}\left(\delta_{A}, \delta_{B}, \delta_{C}\right)$ \\
\hline$[16,4,6]$ & {$[6,1,6]$} & {$[6,0, \infty]$} & {$[[1336,4,6]]$} \\
{$[20,5,8]$} & {$[8,1,8]$} & {$[8,0, \infty]$} & {$[[3100,5,8]]$} \\
{$[24,6,10]$} & {$[10,1,10]$} & {$[10,0, \infty]$} & {$[[5964,6,10]]$} \\
\hline \hline
\end{tabular}




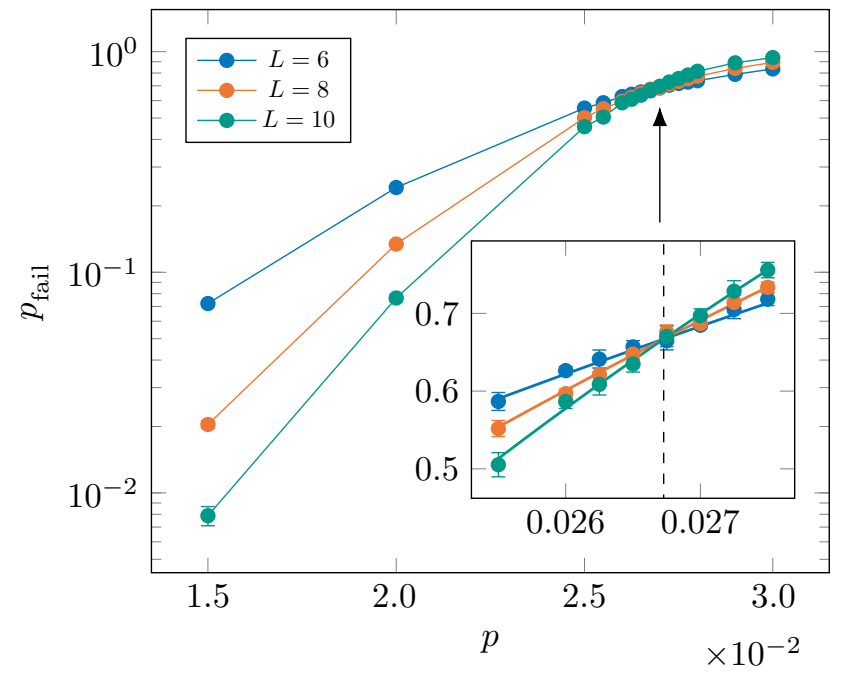

FIG. 4. Threshold plot for the family of nontopological 3D product codes listed in Table III after 16 rounds of single-shot error correction using the $\mathrm{BP}+\mathrm{OSD} \times 2$ decoder. The simulation results suggest a threshold at $2.7 \%$. The error bars show the $95 \%$ confidence intervals $p_{\text {fail }}=\hat{p}_{\text {fail }} \pm 1.96 \sqrt{p_{\text {fail }}\left(1-p_{\text {fail }}\right) / \eta}$, where $\eta$ is the number of Monte Carlo trials.

qubits. An interesting feature of product-code constructions is that they can be used to interpolate between completely local topological codes and random quantum LDPC codes, as explored for the 2D setting in Ref. [56].

To numerically benchmark the single-shot performance of the nontopological code family listed in Table III, we simulate error correction under the two-stage decoder. The strategy we employ is $\mathrm{BP}+\mathrm{OSD} \times 2$, for which both stage1 and stage- 2 decoding use the BP+OSD decoder. The MWPM and BP+OSD strategy used for the surface and toric codes would not work in this setting, as nontopological codes do not have the looplike metacheck syndromes required for MWPM decoding. The simulation results are summarized by Fig. 4, which shows a sustained threshold for the $\mathcal{C}\left(\delta_{A}, \delta_{B}, \delta_{C}\right)$ family of nontopological codes in the region of $2.7 \%$. This result demonstrates that the BP+OSD decoding strategy can be used to decode new 3D product codes and achieve performance close to that of established codes such as the 3D surface and toric codes.

\section{CONCLUSION}

In this paper, we investigate single-shot decoding of 3D product codes. We gave a formal definition of confinement in quantum codes and proved that all 3D product codes have confinement for $Z$ errors. We also prove that confinement is sufficient for single-shot error correction against adversarial noise. This is a strengthening of the result of Campbell [18], who showed that a property called soundness is sufficient for single-shot error correction, in that soundness implies confinement but the converse is not true. Remarkably, there are important classes of codes, such as quantum expander codes, which have confinement but not soundness. Further to that, we prove that codes with linear confinement, and so expander codes, do have a single-shot threshold for local stochastic noise. The obvious open problem arising from our work is how to extend our findings for linear confinement to the superlinear case. Is confinement, in general, a sufficient condition for quantum-LDPC codes to exhibit a single-shot threshold? If not, what other requirements should a code satisfy to ensure the existence of a single-shot threshold?

We simulate single-shot error correction for a variety of 3D product codes, concentrating on 3D toric and surface codes. Using MWPM and BP+OSD, we achieve the best known code-capacity error threshold and sustainable single-shot error threshold for this code family (for phaseflip noise). Our results strongly suggest that the bottleneck of two-stage decoders is the first stage where the noisy syndrome is repaired. For the 3D toric code, the optimal threshold of the syndrome-repair step is 3.3\% [33], whereas the optimal threshold of the entire decoding problem is $11.0 \%$ [35]. This implies that two-stage decoders can never achieve optimal performance in these codes, so perhaps other single-shot decoding methods ought to be investigated in future.

We also simulate single-shot error correction for a family of nontopological 3D product codes, using $\mathrm{BP}+\mathrm{OSD}$ for both decoding steps. We achieve performance very close to that of the 3D toric and surface codes, which indicates that $\mathrm{BP}+\mathrm{OSD}$ is a high-performance single-shot decoder. Furthermore, the versatility of BP+OSD means that we expect it to work as a single-shot decoder for general LDPC 3D product codes. We leave confirmation of this to future work, and we conjecture that BP+OSD will achieve good performance for other classes of quantum-LDPC codes such as topological fracton codes $[61,62]$.

\section{ACKNOWLEDGMENTS}

This work is supported by the Engineering and Physical Sciences Research Council [Grants No. EP/P510270/1 (J.R.S.) and No. EP/M024261/1 (E.T.C. and J.R.)]. J.R. and E.T.C. were supported by the QCDA project (EP/R043825/1) which has received funding from the QuantERA ERA-NET Cofund in Quantum Technologies implemented within the European Union's Horizon 2020 Programme. J.R. also acknowledges funding from BMBF (RealistiQ) and the DFG (CRC 183). M.V. thanks Aleksander Kubica and Nikolas Breuckmann for illuminating discussions. We thank Rui Chao for comments on an early version of the paper. Research at Perimeter Institute is supported in part by the Government of Canada through the Department of Innovation, Science and Economic Development Canada and by the Province of Ontario through the Ministry of Colleges and Universities. This research was 
enabled in part by support provided by Compute Ontario [63] and Compute Canada [64]. This work was completed while E.T.C. was at the University of Sheffield.

\section{APPENDIX A: LINEAR CONFINEMENT AND SINGLE-SHOT THRESHOLD}

We present the stochastic shadow decoder, a variant of the (adversarial) shadow decoder described in Definition 3 , and prove that it succeeds in correcting errors that have connected components that are sufficiently sparse and of bounded size, both on the syndrome and the qubits (Lemma 6). Theorem 2 will then follow from Lemma 6 on the performance of the stochastic shadow decoder: a family of codes with good linear confinement has a single-shot threshold under the local stochastic noise model.

This appendix is organized as follows. After fixing some graph-theory notation in Appendix A 1, we introduce a novel weight function for node sets in a graph, the closeness function, Appendix A 2. We prove that the closeness weight function preserves confinement and that the stochastic shadow decoder can be used on confined codes to keep the closeness of error under control (Appendix A 3). Crucially, the closeness weight function characterizes the structure of local stochastic errors better that the Hamming weight does, as some classic results in percolation theory show. We conclude, in Appendix A 4, by showing that a family of codes with good linear confinement has a sustainable single-shot threshold (Theorem 1). Our proof is built on the results in Refs. [22,65], where the authors prove that expander codes (which have linear confinement) have a single-shot threshold when decoded via the small-set flip decoder.

\section{Notation and preliminaries}

Given a stabilizer code on $n$ qubits with stabilizer group $\mathcal{S} \subseteq \mathcal{P}_{n}$, we associate to it two graphs: $\left(\mathcal{G}_{q}, \sim_{q}\right)$, the qubit graph, and $\left(\mathcal{G}_{s}, \sim_{s}\right)$, the syndrome graph. The set of nodes are $\mathcal{G}_{q}$, the $n$ qubits, and $\mathcal{G}_{s}$, a generating set of the stabilizer group $\mathcal{S}$ [66]. The adjacency relations $\sim_{q}$ and $\sim_{s}$ are defined as

$$
\begin{aligned}
q_{1} \sim_{q} q_{2} & \Leftrightarrow \exists s \in \mathcal{G}_{s} \text { such that }\left\{q_{1}, q_{2}\right\} \subseteq \operatorname{supp}(s), \\
s_{1} \sim_{s} s_{2} & \Leftrightarrow \exists q \in \mathcal{G}_{q} \text { such that } q \in \operatorname{supp}\left(s_{1}\right) \cap \operatorname{supp}\left(s_{2}\right) ;
\end{aligned}
$$

where the support $\operatorname{supp}(s)$ of a Pauli operator $s$ in $\mathcal{P}_{n}$ is the set of all the qubits on which its action is nontrivial. We use lowercase symbols for Pauli operators in $\mathcal{P}_{n}$ and the corresponding uppercase symbol to indicate its support, e.g., $E:=\operatorname{supp}(e)$. We use the term error to refer interchangeably to a Pauli operator or its support, in particular given two Pauli operators $e_{1}$ and $e_{2}$ we use the symbol + to indicate the support of the product operator $e_{1} \cdot e_{2}$ [67], so that

$$
E_{1}+E_{2}=\operatorname{supp}\left(e_{1} \cdot e_{2}\right) .
$$

In this picture, the syndrome $\sigma(\cdot)$ maps the set of Pauli operators on $n$ qubits $\mathcal{P}_{n}$ into the power set of $\mathcal{G}_{s}$,

$$
\begin{aligned}
\sigma: \mathcal{P}_{n} & \longrightarrow \mathscr{P}\left(\mathcal{G}_{s}\right) \\
e & \longrightarrow\left\{s \in \mathcal{G}_{s}: s e=-e s\right\} .
\end{aligned}
$$

We define the neighbor map $\Gamma$ as

$$
\begin{aligned}
\Gamma: \mathcal{P}_{n} & \longrightarrow \mathscr{P}\left(\mathcal{G}_{s}\right) \\
e & \longrightarrow\left\{s \in \mathcal{G}_{s}: \operatorname{supp}(s) \cap E \neq \emptyset\right\} .
\end{aligned}
$$

With slight abuse of terminology, we call syndrome any element of $\mathscr{P}\left(\mathcal{G}_{s}\right)$, even when such a set does not belong to the image of $\sigma$. When referring to an error as a set $E$, it is always to be intended as corresponding to a fixed Pauli operator $e \in \mathcal{P}_{n}$ such that $E:=\operatorname{supp}(e)$. We write interchangeably $\sigma(e) / \sigma(E)$ and $\Gamma(e) / \Gamma(E)$ to indicate the image, via the syndrome map and the neighbor map, respectively, of the Pauli error $e$.

Given two syndromes sets in $\mathcal{G}_{s}$ we use the symbol + to indicate their symmetric difference. It is easy to verify that the map $\sigma(\cdot)$ preserves the + operation (i.e., it is linear):

$$
\sigma\left(e_{1} \cdot e_{2}\right)=\sigma\left(E_{1}+E_{2}\right)=\sigma\left(E_{1}\right)+\sigma\left(E_{2}\right) .
$$

Moreover, the image via $\sigma$ of disjoint nonconnected sets is disjoint. In fact, if $E_{1}, E_{2}$ are two disjoint nonconnected sets in $\mathcal{G}_{q}$ and we suppose that their syndrome sets are not disjoint we find a contradiction. Let $\hat{s}$ be a stabilizer in $\sigma\left(E_{1}\right) \cap \sigma\left(E_{2}\right)$. By definition of $\sigma$, this entails that $e_{1}$ and $e_{2}$ both anticommute with $\hat{s}$, which is equivalent to saying that their supports have odd overlap with $\operatorname{supp}(\hat{s})$. In particular, there exists $q_{i} \in E_{i}$ such that $q_{i} \in \operatorname{supp}(\hat{s})$ and, by the definition of the adjacency relation $\sim_{q}, q_{1} \in E_{1}$ and $q_{2} \in E_{2}$ would be connected via $\hat{s}$, against the assumption. Note that, in general the image via the syndrome map $\sigma(\cdot)$ of a connected set needs not to be connected. However, the neighbor function $\Gamma(\cdot)$ maps connected sets into connected sets. We make use of these properties in Appendix A 3.

\section{The closeness weight function}

When errors are local stochastic it can be handy to use definitions of weight other than the cardinality and Hamming weight. For instance, the authors in Ref. [22] define the quantities of Definition 6 and study a related notion of percolation to understand the tolerance to errors of a given connected graph.

Definition 6 ( $\alpha$ subsets, $\operatorname{MaxConn}_{\alpha}(E)$ [22]). An $\alpha$ subset of a set $E \subseteq \mathcal{G}_{q}$ is a set $K$ such that $|K \cap E| \geq \alpha|K|$. The maximum size of a connected $\alpha$ subset of $E$ is denoted by $\operatorname{MaxConn}_{\alpha}(E)$. 
We here introduce a conceptual cousin to $\operatorname{MaxConn}_{\alpha}(E)$, the $\beta$ closeness of an error set $E$, and prove that it is a welldefined weight function (see Lemma 3 ). We do not explicitly detail the relations between $\alpha$ subsets and closeness here. However, we implicitly use them, as our percolation results and ultimately the proof of Theorem 1 heavily rely on those relations and the proofs in Refs. [22,65].

Definition 7 ( $\beta$ closeness). Let $\mathcal{G}$ be a connected graph, i.e., a graph in which there exist a path between any two of its nodes. Given a subset $E$ of nodes and a positive integer $\beta$, we define its $\beta$ closeness as the quantity:

$$
\|E\|_{\beta}:=\max \{|K \cap E|: K \text { is connected, }|K|=\beta\} .
$$

We call any connected subset of $\beta$ nodes a $\beta$ patch and any $\beta$ patch $K$ such that $|K \cap E|=\|E\|_{\beta}$ maximal patch for $E$.

Since we are interested in the $\beta$ closeness of error sets on a qubit graph $\mathcal{G}_{q}$, it is natural to introduce the notion of reduced $\beta$ closeness.

Definition 8. Given a qubit error set $E \subseteq \mathcal{G}_{q}$, its reduced $\beta$ closeness $\|E\|_{\beta}^{\text {red }}$ is defined as

$$
\begin{gathered}
\|E\|_{\beta}^{\mathrm{red}}:=\min \left\{\|E+T\|_{\beta}: \sigma(E+T)=\sigma(E),\right. \\
\left.T=\operatorname{supp}(t) \text { for some } t \in \mathcal{P}_{n}\right\} .
\end{gathered}
$$

Crucially, we see in Lemma 5 that the closeness function preserves confinement. As a consequence, we can build a variant of the shadow decoder (Definition 9) that succeeds in correcting errors of small reduced closeness.

We now prove some basic properties of the $\beta$-closeness weight function $\|\cdot\|_{\beta}$ on a connected graph $\mathcal{G}$.

Lemma 3. Let $\mathcal{G}$ be a connected graph and denote by $|\mathcal{G}|$ the number of its nodes. For any positive integer $\beta<|\mathcal{G}|$, the following hold:

(i) $\|\cdot\|_{\beta} \leq|\cdot|$;

(ii) $\|\cdot\|_{\beta} \leq \beta$; the equality holds if and only if the considered set of nodes has a connected component of size at least $\beta$; conversely, if $\|\cdot\|_{\beta}<\beta$ then the connected components of the set all have size less than $\beta$;

(iii) it is positive: $\|E\|_{\beta} \geq 0$ and equality holds if and only if $E=\emptyset$;

(iv) it satisfies the triangle inequality: for any $E_{1}, E_{2}$, $\left\|E_{1} \cup E_{2}\right\|_{\beta} \leq\left\|E_{1}\right\|_{\beta}+\left\|E_{2}\right\|_{\beta}$.

(v) it is monotonic: if $E_{1} \subseteq E_{2}$ then $\left\|E_{1}\right\|_{\beta} \leq\left\|E_{2}\right\|_{\beta}$;

In the following, let $K \subseteq \mathcal{G}$ be a maximal $\beta$ patch for $E$, i.e., $\|E\|_{\beta}=|K \cap E|$.

(i) $\|E\|_{\beta}=|K \cap E| \leq|E|$. (ii) $|K \cap E| \leq|K|=\beta$. Equality holds if and only if $K \cap E=K \subseteq E$, which entails that $E$ has a connected component of size at least $\beta$, since $K$ is connected.

(iii) If $E$ is nonempty then there exists at least one node $g \in E$. Since $\mathcal{G}$ is connected, for any integer $1 \leq$ $\beta \leq|\mathcal{G}|$ there exists a $\beta$ patch that contains $g$ so that $\|E\|_{\beta} \geq 1$.

(iv) Let $J$ be any $\beta$ patch in $\mathcal{G}$. The following hold:

$$
\begin{aligned}
\left|J \cap\left(E_{1} \cup E_{2}\right)\right|= & \left|\left(J \cap E_{1}\right)\right|+\left|\left(J \cap E_{2}\right)\right|+ \\
& -\left|J \cap\left(E_{1} \cap E_{2}\right)\right| \\
\leq & \left|J \cap E_{1}\right|+\left|J \cap E_{2}\right| \\
\leq & \left\|E_{1}\right\|_{\beta}+\left\|E_{2}\right\|_{\beta} .
\end{aligned}
$$

Since this holds for any $\beta$ patch, we obtain

$$
\left\|E_{1} \cup E_{2}\right\|_{\beta} \leq\left\|E_{1}\right\|_{\beta}+\left\|E_{2}\right\|_{\beta} .
$$

(v) Let $K_{1}, K_{2}$ be maximal $\beta$ patches for $E_{1}$ and $E_{2}$, respectively. Then

$$
\begin{aligned}
\left|K_{1} \cap E_{1}\right| & \leq\left|K_{1} \cap E_{2}\right| \quad \text { because } E_{1} \subseteq E_{2}, \\
& \leq\left|K_{2} \cap E_{2}\right| \quad \text { by maximality of } K_{2},
\end{aligned}
$$

which yields $\left\|E_{1}\right\|_{\beta} \leq\left\|E_{2}\right\|_{\beta}$.

Lemma 2 below states that there exists a canonical form for maximal $\beta$ patches of an error set $E$. Roughly speaking, a canonical $\beta$ patch $K$ will be made up of some entire connected components of $E$, plus at most one connected proper subset of a connected component of $E$, and some other nodes not in $E$ (see Fig. 5). The existence of a canonical $\beta$ patch is key in proving that the closeness function preserves confinement in the sense explained by Lemma 5 .

Lemma 4 (Canonical $\beta$ patch). For any error $E$ on a qubit graph $\mathcal{G}$ there exists a maximal $\beta$ patch $T$ such that, for all but one connected component $E_{i}$ of $E$, the following holds:

$$
\text { either } E_{i} \subseteq T \text { or } E_{i} \cap T=\emptyset .
$$

In other words, if $E_{1}, \ldots, E_{m}$ are the connected components of E, reordering if necessary, there exists an index v such that

$$
\begin{array}{ll}
\left|T \cap E_{i}\right|=\left|E_{i}\right| & \text { if } i<v, \\
\left|T \cap E_{i}\right| \leq\left|E_{i}\right| & \text { if } i=v, \\
\left|T \cap E_{i}\right|=0 & \text { if } i>v .
\end{array}
$$

We call any such $T$ a canonical $\beta$ patch for the set $E$.

Let $J$ be any maximal $\beta$ patch for $E$, i.e., $J$ is connected, has size $\beta$ and $|J \cap E|=\|E\|_{\beta}$. Starting from $J$ we build a 
(a)

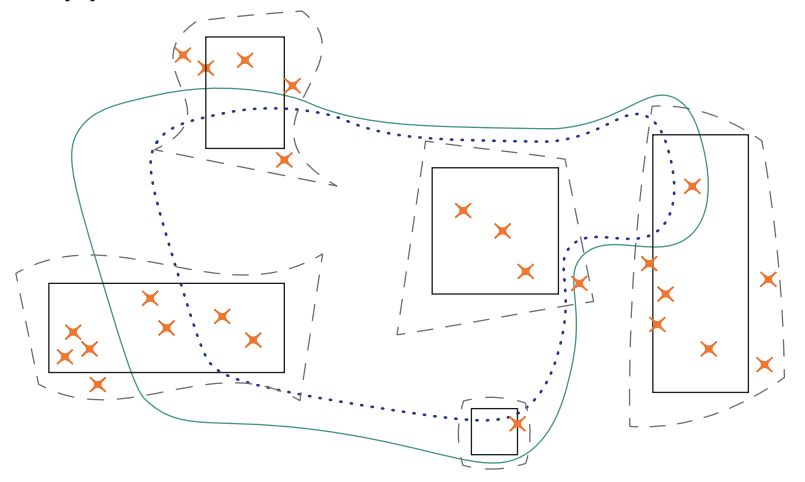

(b)

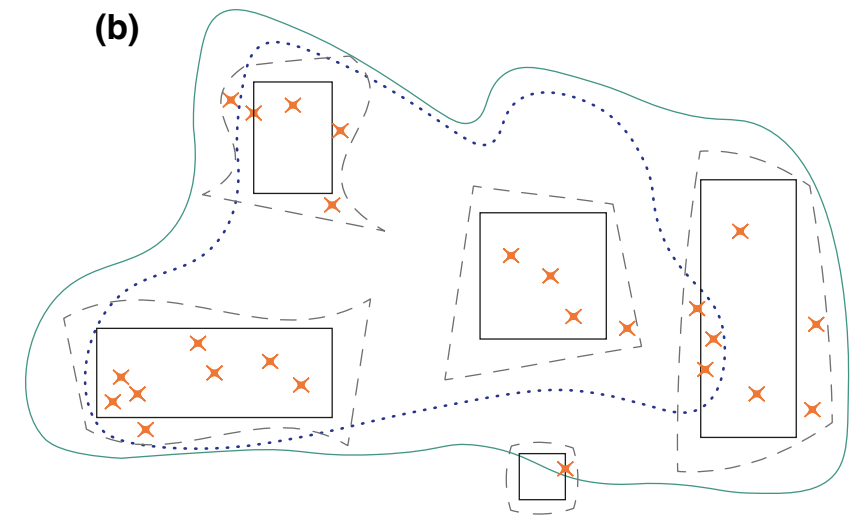

FIG. 5. Graphical representation of patches on a graph. To help the visualization we imagine the qubit graph and the syndrome graph to be superimposed. Black rectangles: connected components of the error $E_{1}, \ldots, E_{5}$. Dashed grey lines: neighbour sets $\Gamma\left(E_{i}\right)$ of the underlying rectangle and error component. Orange crosses: syndrome nodes in $\sigma\left(E_{i}\right)$. Dotted blue curve: $t$ patches on the qubit graph. Green curve: $\omega t$ patches on the syndrome graph. In (a) the patches are generic while in (b) the dotted and error patch is a canonical patch for the error. The importance of the canonical form for a patch is highlighted in the differences between the patches in (a),(b). We observe how the crosses and syndrome nodes $\sigma\left(E_{i}\right)$ are scattered inside the dashed curve and neighbor set $\Gamma\left(E_{i}\right)$. For this reason, in order to group enough syndrome nodes inside a patch of bounded size, we need some care in the choice of the error nodes. When we include entire connected components of the error in a patch in $\mathcal{G}_{q}$, we are able to build a patch in $\mathcal{G}_{s}$, which includes entire neighbor sets and, as a consequence, all the corresponding syndrome nodes. In fact, even if we assume that the dotted blue and error patches in (a),(b) have the same size, when we enlarge them by a factor of $\omega$ to build the green and syndrome patch, we obtain dramatically different results. In (a) since the dotted and error patch contains several incomplete components, the corresponding green and syndrome patch contains incomplete portions of the dashed and neighbor sets $\Gamma\left(E_{i}\right)$. Hence, we have no guarantee on the number of crosses and syndrome nodes included in the patch. In (b) the dotted blue patch is a canonical patch for the error. We can see how the green and syndrome patch entirely contains the dashed and neighbor sets of all but one component of the error contained in the dotted blue and qubit patch. In this way we have the certainty to include in the green and syndrome patch a sufficient number of crosses and syndrome nodes to ensure confinement.

set $T$ with the desired form. Write $J \cap E$ as disjoint union of connected sets:

$$
J \cap E=J_{1} \sqcup \cdots \sqcup J_{v}
$$

We call these $J_{i}$ 's patch-error components. Let $E_{1} \ldots E_{\mu}$ be the connected components of the error $E$. We recall that a connected component $E_{i}$ of $E$ is a connected set, which is connected to no additional nodes in $E \backslash E_{i}$. We say that $E_{i}$ is incomplete with respect to $J$ if it has nontrivial overlap with $J$ but it is not entirely contained in $J$, i.e.,

$$
J \cap E_{i} \neq \emptyset \& E_{i} \nsubseteq J \Rightarrow\left|J \cap E_{i}\right|<\left|E_{i}\right| .
$$

Note that it can be the case for two disjoint (but internally connected) error-patch components $J_{i_{1}}$ and $J_{i_{2}}$ to overlap with the same incomplete error component $E_{i^{\prime}}$.

We consider a metagraph $\mathfrak{G}$ whose metanodes are connected sets in $\mathcal{G}$ and metaedges are paths in $\mathcal{G}$. Because the error-patch components are both internally and reciprocally connected in $J$, there exists a meta spanning tree $\mathcal{T} \subseteq \mathfrak{G}$ whose $v$ nodes $\mathcal{J}_{i}$ are the error-patch components $J_{i}$ and whose metaedges $\varepsilon_{i j}$ are formed by minimum length paths in $\mathcal{G}$ between the $J_{i}$ 's with nodes in $J \backslash E$. In the following we indicate with $\mathcal{T}, \mathcal{J}_{i}$ and $\varepsilon_{i j}$ the metatree, its metanodes, and its metaedges and with $T, J_{i}$, and $e_{i, j}$ the corresponding sets of nodes in $\mathcal{G}$. Note that, by this identification, $T$ has at most $\beta$ nodes. We now show how to modify the metatree $\mathcal{T}$ so that the corresponding set of nodes $T$ in $\mathcal{G}$ is canonical for $E$. We do this in two steps: the balancing and the enlargement step.

\section{a. BALANCING}

We show by induction on the number $v$ of the metanodes $\mathcal{J}_{i}$ 's that it is possible to modify $\mathcal{T}$ so that the corresponding set of nodes $T \subseteq \mathcal{G}$ satisfies conditions (A1) on its overlap with the connected components of $E$.

$v=1:$ the thesis is trivially verified.

$v>1$ : if $J$ is not canonical for $E$ then $E$ must have at least two incomplete components with respect to the patch $J$. Let $\mathcal{J}_{\ell}$ be a metaleaf of $\mathcal{T}$ and $J_{\ell}$ its corresponding subset of nodes in $\mathcal{G}$. We iteratively remove from $T$ the nodes of $J_{\ell}$, both preserving connectivity of $T$ and the size of $T \cap E$.

For any node $q_{\lambda}$ in $J_{\ell}$, we choose a node $q_{\chi}$ such that the following holds:

i. $q_{x}$ belongs to some incomplete component of the error disjoint from $J_{\ell}: q_{\chi} \in E_{\chi}$ and $E_{\chi} \cap J_{\ell}=\emptyset$; 
ii. $q_{\chi}$ is a new node, i.e., it does not belong to $J: q_{\chi} \in$ $\mathcal{G} \backslash J$

iii. $q_{x}$ is connected to at least one node in some errorpatch component other from $J_{\ell}: q_{\chi} \sim_{q} q_{\chi^{\prime}}, q_{\chi^{\prime}} \in J_{\chi}$ for some $\chi \neq \ell$.

We remove $q_{\lambda}$ from $J$ and add $q_{\chi}$ to $J$, and thereby update $T$ accordingly. This process terminates when either (a) we are not able to find such a new node $q_{\chi}$ or (b) there are no more nodes $q_{\lambda}$ in $J_{\ell}$.

Case (a) entails that $E$ has at most one incomplete component with respect to $T$. In fact, if $E$ had an incomplete component $E_{\chi}$ disjoint from $J_{\ell}$ such a node $q_{\chi}$ always exists. As a consequence, if we are not able to find a new error node to enlarge one of the error-patch components $J_{\chi} \neq J_{\ell}$ the only incomplete component of $E$ must be the one relative to $J_{\ell}$. The updated node set $T$ has the desired property, provided that we had removed nodes $q_{\lambda}$ from $J_{\ell}$ preserving connectivity (for instance, considering a spanning tree for nodes in $J_{\ell}$ and iteratively removing leaves). If case (b) is verified, we remove from $\mathcal{T}$ all the metaedges that are incident to $\mathcal{J}_{\ell}$. The updated metatree $\mathcal{T}$ derived from the updated set $T$ has $v-1$ metanodes. By the induction hypothesis, it can be modified to obtain the desired form.

In other words, we pick a metaleaf of $\mathcal{T}$ and we either remove part of its nodes [case (a)] or all of them [case (b)]. We preserve the quantity $|T \cap E|$ by adding new error nodes to some different error-patch component that overlaps with an incomplete component of the error $E$. By choosing a leaf, we are able to preserve the connectivity of $\mathcal{T}$ and thus the connectivity of the corresponding node sets $T$.

We iterate this procedure over metaleaves of $\mathcal{T}$ until the overlap of the corresponding set $T$ in $\mathcal{G}$ and the error set $E$ has the desired form.

\section{b. ENLARGEMENT}

By contradiction, we prove that it is possible to add nodes to the set $T$ corresponding to the balanced metatree $\mathcal{T}$ so that it is connected, it has size exactly $\beta$ and $\mid T \cap$ $E \mid=\|E\|_{\beta}$. First note that during the balancing procedure, the number $|T \cap E|$ remains constant and the following holds:

$$
|T \cap E|=\sum_{i=1}^{v}\left|J_{i}\right|=|J \cap E|=\|E\|_{\beta} .
$$

Moreover, the initial tree is connected and the balancing procedure preserves connectivity. However, we only have an upper bound on the size of $T$. In fact, if $\mathcal{T}$ is the initial metatree and $T$ is its corresponding subgraph in $\mathcal{G}$, it holds $T \subseteq J$ and therefore $|T| \leq \beta$. During the balancing step the size of $T$ could decrease when we remove nodes of $e_{i j}$, belonging to a metaedge $\varepsilon_{i j}$. Thus, in general, after the balancing step for the weight of $T$ holds:

$$
|T| \leq \beta .
$$

If $|T|=\beta$, then $T$ is a $\beta$ patch with maximum overlap with $E$ and, by balancing, it is canonical. If $|T|<\beta$, then there must exist at least $\beta-\|E\|_{\beta}$ nodes in $\mathcal{G} \backslash(E \cup T)$ that are connected to $T$. In fact, a connected proper subset can always be enlarged in a connected graph. If the only way to enlarge $T$ to a $\beta$ patch were by adding nodes in $E$, then we would have found a $\beta$ patch whose overlap with $E$ has size greater than its $\beta$ closeness, which contradicts the definition of $\|E\|_{\beta}$. In conclusion, any of such enlargements of the tree $T$ is a canonical $\beta$ patch for $E$.

\section{Confinement and stochastic shadow decoder}

Here, we first prove that the closeness function preserves confinement, as Lemma 5 states. Then, we present the stochastic shadow decoder (Definition 9) and prove, in Lemma 6, that it succeeds in correcting errors of small enough closeness. These findings, together with the percolation results of Appendix A 4, will yield the proof of the existence of a single-shot threshold for codes with linear confinement.

Lemma 5 (Closeness preserves confinement). Consider a code with qubit degree at most $\tilde{\omega}$ and $(t, f)$ confinement, where $f$ is convex. Then, for any error $E$ with $\|E\|_{t}^{\text {red }} \leq$ $(t / 2)$, the following holds:

$$
f\left(\|\sigma(E)\|_{\omega t}\right) \geq\|E\|_{t}^{\mathrm{red}},
$$

where $\omega=\tilde{\omega}+1$.

To ease the notation, let $F$ be an error set such that $\sigma(E)=\sigma(F),\|F\|_{t}=\|E\|_{t}^{\text {red }}$. If $F_{1}, \ldots, F_{\mu}$ are the connected components of $F$, by Lemma 4 there exists a canonical patch $K$ for $F$ such that

$$
\begin{array}{ll}
\left|K \cap F_{i}\right|=\left|F_{i}\right| & \text { if } \quad i<v, \\
\left|K \cap F_{i}\right| \leq\left|F_{i}\right| & \text { if } \quad i=v, \\
\left|K \cap F_{i}\right|=0 & \text { if } \quad i>v .
\end{array}
$$

for some $v \leq \mu+1$.

First, we prove that there exists a $\omega t$ patch $J$ in the syndrome graph $\mathcal{G}_{s}$ such that it contains the syndrome of the connected components $F_{1}, \ldots, F_{v}$ of the error, which intersect the canonical patch $K$ :

$$
\bigsqcup_{i=1}^{v}\left[\sigma\left(F_{i}\right)\right] \subseteq J
$$

Then, we prove that such a patch $J$ has overlap with $\sigma(F)$ of Hamming weight large enough to ensure confinement 
with respect to the closeness function:

$$
f\left(\|\sigma(F)\|_{\omega t}\right) \geq\|F\|_{t} .
$$

We then find the desired bound on $E$ using the initial assumptions $\sigma(F)=\sigma(E)$ and $\|F\|_{t}=\|E\|_{t}^{\text {red }}$.

\section{a. EXISTENCE OF $J$}

We build a $\omega t$ patch $J$ on $\mathcal{G}_{s}$ as follows. We define $J$ as the disjoint union of the (at most) $\tilde{\omega}\left|F_{i}\right|$ connected nodes $\Gamma\left(F_{i}\right)$ :

$$
J=\bigsqcup_{i=1}^{v} \Gamma\left(F_{i}\right) .
$$

Let $\pi$ be the set of edges of a minimum length path in $K$ that connects all its $v$ disjoint error components $F_{i}$. These edges correspond naturally to a set $\pi_{s} \subseteq \mathcal{G}_{s}$ if we associate to the edge $\left(q_{1}, q_{2}\right)$, the corresponding stabilizer in $\mathcal{G}_{s}$, remembering that

$$
q_{1} \sim_{q} q_{2} \Leftrightarrow\left\{q_{1}, q_{2}\right\} \subseteq \operatorname{supp}(s), \quad s \in \mathcal{G}_{s} .
$$

Under this identification, importantly, adjacent edges are mapped into neighboring syndrome nodes. We add the set $\pi_{s}$ to $J$. As a result, $J$ is now connected. For the size of $J$, the following holds:

$$
|J| \leq \tilde{\omega} \sum_{i=1}^{\nu}\left|F_{i}\right|+\left|\pi_{s}\right| .
$$

By hypothesis, $t / 2 \geq\|F\|_{t}=|K \cap F|$ and because $K$ is canonical for $F$, i.e., $|K \cap F| \leq \sum_{i=1}^{v}\left|F_{i}\right|$, we have

$$
\sum_{i=1}^{v-1}\left|F_{i}\right| \leq \frac{t}{2}
$$

Combining property (ii) of the closeness weight function and the assumption $\|F\|_{t} \leq(t / 2)$, yields, for any $i$, and $v$ in particular,

$$
\left|F_{i}\right| \leq \frac{t}{2}
$$

Since $\pi$ has edges in $K, \pi_{s}$ has size at most $|K|$, i.e.,

$$
\left|\pi_{s}\right| \leq t
$$

Adding up, we obtain

$$
|J| \leq \omega t,
$$

where $\omega=\tilde{\omega}+1$. By enlarging $J$ if necessary to include exactly $\omega t$ nodes, and remembering that by construction it is connected, we find that $J$ is a $\omega t$ patch in $\mathcal{G}_{s}$, as desired.

\section{b. OVERLAP OF $\boldsymbol{J}$ WITH THE ERROR SYNDROME}

Equation (A2) entails in particular that any connected error component $F_{1}, \ldots, F_{v}$ that has nontrivial overlap with the patch $K$, has size smaller than $t$ and therefore it has confinement:

$$
f\left(\left|\sigma\left(F_{i}\right)\right|\right) \geq\left|F_{i}\right| .
$$

Because $\sigma$ maps disjoint sets of $\mathcal{G}_{q}$ in disjoint sets of $\mathcal{G}_{s}$,

$$
\begin{aligned}
\sigma\left(\bigsqcup_{i=1}^{v} F_{i}\right) & =\bigsqcup_{i=1}^{v} \sigma\left(F_{i}\right) \\
\Rightarrow\left|\sigma\left(\bigsqcup_{i=1}^{v} F_{i}\right)\right| & =\sum_{i=1}^{v}\left|\sigma\left(F_{i}\right)\right| .
\end{aligned}
$$

Thus, applying $f$ to each term of the summation of Eq. (A4) we have

$$
\sum_{i=1}^{v} f\left(\left|\sigma\left(F_{i}\right)\right|\right) \geq \sum_{i=1}^{v}\left|F_{i}\right| .
$$

For the left-hand side of Eq. (A5), using convexity of $f$ we obtain

$$
f\left(\sum_{i=1}^{v}\left|\sigma\left(F_{i}\right)\right|\right) \geq \sum_{i=1}^{v} f\left(\left|\sigma\left(F_{i}\right)\right|\right),
$$

for the right-hand side of Eq. (A5) instead, since $K$ is canonical for $F$, it holds that

$$
\sum_{i=1}^{v}\left|F_{i}\right| \geq|K \cap F|,
$$

Combining these two bounds for Eq. (A5) yields

$$
f\left(\sum_{i=1}^{v}\left|\sigma\left(F_{i}\right)\right|\right) \geq\|F\|_{t} .
$$

To obtain the thesis from Eq. (A6), we just need to substitute the Hamming weight on the left-hand side with the closeness weight $\|\cdot\|_{\omega t}$. By construction, for $J$ it holds that

$$
|J \cap \sigma(F)| \geq \sum_{i=1}^{v}\left|\sigma\left(F_{i}\right)\right| .
$$

Moreover, since $J$ is a $\omega t$ patch:

$$
\|\sigma(F)\|_{\omega t} \geq|J \cap \sigma(F)| .
$$

Using the monotonicity of $f$ and combining Eqs. (A7), (A8), and (A6) yields

$$
f\left(\|\sigma(F)\|_{\omega t}\right) \geq\|F\|_{t} .
$$




\section{c. CONCLUSION}

Because $F$ is an error set equivalent to $E$, i.e., $\sigma(F)=$ $\sigma(E)$, such that $\|F\|_{t}=\|E\|_{t}^{\text {red }}$, we conclude

$$
f\left(\|\sigma(E)\|_{\omega t}\right) \geq\|E\|_{t}^{\mathrm{red}}
$$

for $\omega=\tilde{\omega}+1$.

Lemma 5 in particular entails that the closeness weight is in fact a sensible quantity to look at when dealing with errors on confined codes.

We now introduce the stochastic shadow decoder. The difference between this variant and the one previously presented (Definition 3) is on the weight functions used. While the standard and adversarial shadow decoder tries to minimize the Hamming weight of the residual error, the stochastic shadow decoder attempts to keep under control its closeness.

Definition 9 (Stochastic shadow decoder). The stochastic shadow decoder has variable parameters $0<\alpha \leq 1$, and $0<\beta, \gamma \in \mathbb{Z}$. Given an observed syndrome $S=\sigma(E)+$ $S_{e}$ where $S_{e} \subseteq \mathcal{G}_{s}$ is the syndrome error, the stochastic shadow decoder of parameters $(\alpha, \beta, \gamma)$ performs the following two steps:

1. Syndrome repair: find $S_{r}$ of minimum $\gamma$ closeness $\left\|S_{r}\right\|_{\gamma}$ such that $S+S_{r}$ belongs to the $(\alpha, \beta)$ shadow of the code, where

$$
(\alpha, \beta) \text { shadow }=\left\{\sigma(E) \text { such that }\|E\|_{\beta} \leq \alpha \beta\right\} .
$$

2. Qubit decode: find $E_{r}$ of minimum $\beta$ closeness $\left\|E_{r}\right\|_{\beta}$ such that $\sigma\left(E_{r}\right)=S+S_{r}$.

We call $R=E+E_{r}$ the residual error.

We have the following promise on the stochastic shadow decoder, which mirrors the results of Lemma 1 for the adversarial shadow decoder.

Lemma 6. Consider a stabilizer code that has $(t, f)$ confinement and qubit degree $\leq \omega-1$. Provided that the original error pattern $E$ has $\|E\|_{t}^{\text {red }} \leq t / 2$, on input of the observed syndrome $S=\sigma(E)+S_{e}$, the residual error $R$ left by the stochastic shadow decoder of parameter $[(1 / 2), t, \omega t]$ satisfies

$$
\|R\|_{t}^{\text {red }} \leq f\left(2\left\|S_{e}\right\|_{\omega t}\right) .
$$

Thanks to Lemma 5, we know that the closeness function preserves confinement. The proof is then a straightforward adaption of the proof of Lemma 1, where the Hamming weight has to be substituted with $\|\cdot\|_{t}$ on error sets and $\|\cdot\|_{\omega t}$ on syndrome sets, respectively. We here briefly report the proof for completeness.
Assume $\|E\|_{t}^{\text {red }} \leq t / 2$, and let $E_{r}$ be the output of the qubit decode step. By construction, it has minimum $t$ closeness among the errors with syndrome $S+S_{r}$, which belongs to the $[(1 / 2), t]$ shadow of the code. In particular, $\left\|E_{r}\right\|_{t} \leq(t / 2)$. We recall that the + operation between two error sets in $\mathcal{G}_{q}$ denotes the support of the product of the two corresponding Pauli operators and, as such, it holds that (see Appendix A 1)

$$
E+E_{r} \subseteq E \cup E_{r} .
$$

By the property of the closeness weight function, this entails

$$
\left\|E+E_{r}\right\|_{t} \leq\left\|E \cup E_{r}\right\|_{t} \leq\|E\|+\left\|E_{r}\right\|_{t} .
$$

The linearity of the syndrome function $\sigma(\cdot)$ yields

$$
\sigma\left(E+E_{r}\right)=\sigma(E)+\sigma\left(E_{r}\right)=S_{e}+S_{r} .
$$

Since $S_{e}$ is a possible solution of the syndrome-repair step $\left\|S_{r}\right\|_{\omega t} \leq\left\|S_{e}\right\|_{\omega t}$ and so,

$$
\begin{aligned}
\left\|S_{e}+S_{r}\right\|_{\omega t} & \leq\left\|S_{e}\right\|_{\omega t}+\left\|S_{r}\right\|_{\omega t} \\
& \leq 2\left\|S_{e}\right\|_{\omega t} .
\end{aligned}
$$

Combining this and the monotonicity of $f$ gives

$$
\left\|E+E_{r}\right\|_{t}^{\mathrm{red}} \leq f\left(2\left\|S_{e}\right\|_{\omega t}\right) .
$$

Lemma 5 tells us that the stochastic shadow decoder succeeds whenever the $t$ closeness of the error is small enough. Importantly then, if we are able to bound the probability of the complement of this event, we could infer an upper bound on the failure probability of our decoder. This is the subject of the next section.

\section{Percolation results and proof of Theorem 2}

We consider error sets $E$ on the qubit graph $\mathcal{G}_{q}$ and error sets $S_{e}$ on the syndrome graph $\mathcal{G}_{s}$ and we assume that the probability of observing a particular error is at most exponential in its size. Formally, we use this error model.

Definition 10 (Local stochastic error). An error set $E$ on a graph $\mathcal{G}$ is local stochastic of parameter $p$ if, for all set of nodes $G \subseteq \mathcal{G}$, the following holds:

$$
\mathbb{P}(G \subseteq E) \leq p^{|G|} .
$$

We then use some results in percolation theory, Lemmas 7 and 8 below, to understand the probability that errors of closeness linear in the patch size (i.e., $\|E\|_{\beta}=\alpha \beta$ for some $0<\alpha \leq 1)$ occur when the noise is local stochastic. 
Lemma 7 (Corollary 28 of Ref. [22]). Let $\mathcal{G}$ be a graph with vertex degree upper bounded by $z$. Then the number $N_{\beta}$ of connected components of size $\beta$ ( $\beta$ patches) satisfies

$$
N_{\beta} \leq|\mathcal{G}| \Phi^{\beta},
$$

where $\Phi=(z-1)\left(1+\frac{1}{z-2}\right)^{z-2}$.

Lemma 8. Let $\mathcal{G}$ be a graph with vertex degree upper bounded by $z$. Let $t$ be a positive integer and $0<\alpha \leq 1$. Then there exists $p_{\text {th }}>0$ such that, for local stochastic errors $E$ of parameter $p<p_{\text {th }}$, we have

$$
\mathbb{P}\left(\|E\|_{t} \geq \alpha t\right) \leq \frac{|\mathcal{G}|}{1-2^{h(\alpha) / \alpha} p}\left(\frac{p}{p_{\text {th }}}\right)^{\alpha t},
$$

where $h(\alpha)=\alpha \log _{2}(1 / \alpha)+(1-\alpha) \log _{2}(1 / 1-\alpha)$ is the binary entropy function.

The proof is a straightforward adaption of the proof of Theorem 17 in Ref. [22]. By expanding the left-hand side of Eq. (A10), we find

$$
\begin{aligned}
\mathbb{P}\left(\|E\|_{t} \geq \alpha t\right) & =\mathbb{P}(\exists K t \text { patch }:|K \cap E| \geq \alpha t) \\
& \leq \sum_{K \text { is a } t \text { patch }} \mathbb{P}(|K \cap E| \geq \alpha t) .
\end{aligned}
$$

Observe that, for a $t$ patch $K$,

$$
\begin{aligned}
\mathbb{P}(|K \cap E| \geq \alpha t) & \leq \sum_{m \geq \alpha t} \sum_{\substack{K^{\prime} \subseteq K \\
\left|K^{\prime}\right|=m}} \mathbb{P}\left(K \cap E=K^{\prime}\right) \\
& \leq \sum_{m \geq \alpha t} \sum_{\substack{K^{\prime} \subseteq K \\
\left|K^{\prime}\right|=m}} \mathbb{P}\left(K^{\prime} \subseteq E\right) \\
& \leq \sum_{m \geq \alpha t} \sum_{\substack{K^{\prime} \subseteq K \\
\left|K^{\prime}\right|=m}} p^{m} \\
& \leq \sum_{m \geq \alpha t}\left(\begin{array}{c}
t \\
m
\end{array}\right) p^{m}
\end{aligned}
$$

By Stirling's approximation [68],

$$
\mathbb{P}(|K \cap E| \geq \alpha t) \leq \frac{\left(2^{h(\alpha) / \alpha} p\right)^{\alpha t}}{1-2^{h(\alpha) / \alpha} p} .
$$

Combining Eqs. (A11), (A12), and Lemma 7 yields

$$
\begin{aligned}
\mathbb{P}\left(\|E\|_{t} \geq \alpha t\right) & \leq N_{t} \frac{\left(2^{h(\alpha) / \alpha} p\right)^{\alpha t}}{1-2^{h(\alpha) / \alpha} p} \\
& \leq \frac{|\mathcal{G}|}{1-2^{h(\alpha) / \alpha} p} \cdot\left(\Phi 2^{h(\alpha)} p^{\alpha}\right)^{t}
\end{aligned}
$$

By imposing the right-hand side to decrease with $t$, we find

$$
p \leq\left(\frac{1}{\Phi 2^{h(\alpha)}}\right)^{\frac{1}{\alpha}}:=p_{\text {th }} .
$$

And in conclusion,

$$
\mathbb{P}\left(\|E\|_{t} \geq \alpha t\right) \leq \frac{|\mathcal{G}|}{1-2^{h(\alpha) / \alpha} p}\left(\frac{p}{p_{\text {th }}}\right)^{\alpha t} .
$$

Finally, we are able to prove that there exists a threshold under which the probability of local stochastic errors to be noncorrectable via the stochastic shadow decoder becomes exponentially small in the system size, provided that the graphs $\mathcal{G}_{s}$ and $\mathcal{G}_{q}$ have bounded degree and linear confinement.

By Lemma 6, the residual error left by the stochastic shadow decoder on a $(t, f)$-confined code is kept under control provided that

$$
\|E\|_{t} \leq \frac{t}{4} \text { and } f\left(2\left\|S_{e}\right\|_{\omega t}\right) \leq \frac{t}{4} .
$$

If the function $f$ is linear, i.e., $f(x)=\kappa x$ for some $\kappa>$ $0 \in \mathbb{Z}$, then conditions (A13) can be written as

$$
\|E\|_{t} \leq \frac{t}{4} \text { and }\left\|S_{e}\right\|_{\omega t} \leq \frac{t}{8 \kappa} .
$$

If the qubit error $E$ is local stochastic of parameter $p$ and the syndrome error $S_{e}$ is local stochastic of parameter $q$, thanks to Lemma 8 , we obtain

$$
\begin{array}{r}
\mathbb{P}\left(\|E\|_{t} \geq t / 4\right) \leq \frac{\left|\mathcal{G}_{q}\right|}{1-2^{4 h\left(\frac{1}{4}\right)} p}\left(\frac{p}{p_{\text {th }}}\right)^{\frac{t}{4}} \\
:=C_{q}\left|\mathcal{G}_{q}\right|\left(\frac{p}{p_{\text {th }}}\right)^{\frac{t}{4}}
\end{array}
$$

and

$$
\begin{gathered}
\mathbb{P}\left(\left\|S_{e}\right\|_{\omega t} \geq \frac{t}{8 \kappa}\right) \leq \frac{\left|\mathcal{G}_{s}\right|}{1-2^{8 \omega \kappa h\left(\frac{1}{8 \omega \kappa}\right)} q}\left(\frac{q}{q_{\mathrm{th}}}\right)^{\frac{t}{8 \omega \kappa}} \\
:=C_{s}\left|\mathcal{G}_{s}\right|\left(\frac{q}{q_{\mathrm{th}}}\right)^{\frac{t}{8 \omega \kappa}}
\end{gathered}
$$

where

$$
p_{\text {th }}:=\left(\frac{1}{\Phi_{q} 2^{h\left(\frac{1}{4}\right)}}\right)^{4} \text { and } q_{\text {th }}:=\left(\frac{1}{\Phi_{s} 2^{h\left(\frac{1}{8 \omega \kappa}\right)}}\right)^{8 \omega \kappa} .
$$

As a result, by Lemma 6, the residual error is correctable except with probability at most

$$
\max \left\{C_{q}\left|\mathcal{G}_{q}\right|\left(\frac{p}{p_{\mathrm{th}}}\right)^{\frac{t}{4}}, C_{s}\left|\mathcal{G}_{s}\right|\left(\frac{q}{q_{\mathrm{th}}}\right)^{\frac{t}{8 \omega \kappa}}\right\} .
$$


In other words, for local stochastic noise of intensity $p \leq p_{\text {th }}$ on the qubits and $q \leq q_{\text {th }}$ on the syndrome, the stochastic shadow decoder has a sustainable single-shot threshold.

We conclude by noting that the assumption of linear confinement is key in the proof of Theorem 2. However, we speculate that the limitations of Theorem 2 are an artefact of our proof and superlinear confinement is a sufficient condition for a family of codes to exhibit a single-shot threshold. In fact, the existence of a threshold $p_{\text {th }}$ and $q_{\text {th }}$ relies on the bounds given in Lemma 8 . There, it is fundamental that the relation between the chosen size of the patch and the size of the overlap with the error is linear [see Eqs. (A11) and (A12)]. In other words, Lemma 8 states that, if we take $\beta$ patches on the error graph and $\gamma$ patches on the syndrome graph, we are able to estimate the probability that errors have closeness less than $\alpha \beta$ and $\tilde{\alpha} \gamma$, respectively. By Eq. (A13), in order to bound the closeness of the residual error left by the stochastic shadow decoder, we need

$$
\left\|S_{e}\right\|_{\gamma} \leq \frac{1}{2} f^{-1}(\alpha \beta) .
$$

As a consequence, combining this with the requirements of Lemma 8, entails

$$
\gamma=\kappa\left(\frac{1}{2} f^{-1}(\alpha \beta)\right),
$$

for some positive constant $\kappa$. In conclusion, building up on the results of Lemma 8, we either need to prove that confinement is preserved if we take on the syndrome graph patches of size linear in $f^{-1}(\alpha \beta)$ or, using our Lemma 5 without modification, that the function is itself linear.

\section{APPENDIX B: QUBIT PLACEMENT ON A 3D LATTICE}

Here we detail how to embed a 3D product code on a cubic lattice, where qubits sit on edges, $Z$ stabilizers on vertices, $X$ stabilizers on faces and metachecks on cells.

Let $C^{0}$ and $C^{1}$ be two vector spaces over $\mathbb{F}$ with basis $\mathcal{B}^{0}=\left\{e_{1}^{0}, \ldots, e_{n}^{0}\right\}$ and $\mathcal{B}^{1}=\left\{e_{1}^{1}, \ldots, e_{m}^{1}\right\}$, respectively. Given a linear map from $C^{0}$ into $C^{1}$, it can be represented as a $m \times n$ matrix $\delta$ over $\mathbb{F}$ such that its action on the elements of the basis $\mathcal{B}^{0}$ is given by

$$
\begin{aligned}
& \delta: C^{0} \longrightarrow C^{1} \\
& e_{i}^{0} \longmapsto \delta e_{i}^{0}=\sum_{\alpha=1}^{m} \delta_{\alpha, i} e_{\alpha}^{1} .
\end{aligned}
$$

Expression (B1) allows us to write the support of vectors in $\delta\left(\mathcal{B}^{0}\right)=\left\{\delta e_{i}^{0}\right\}_{i}$ in a compact form. In fact, the support of $\delta e_{i}^{0}$ is the subset of $\mathcal{B}^{1}$ :

$$
\operatorname{supp}\left(\delta e_{i}^{0}\right)=\left\{e_{\alpha}^{1}: \delta_{\alpha, i} \neq 0\right\}_{\alpha} .
$$

Since basis vectors are uniquely identified by their index, we can compactly write Eq. (B1) as a relation $*$ on the set of indices of the basis $\mathcal{B}^{0}$ and $\mathcal{B}^{1}$ :

$$
\begin{aligned}
\{1, \ldots, n\} & \longrightarrow\{1, \ldots, m\} \\
\kappa & \longrightarrow \kappa^{*}
\end{aligned}
$$

where

$$
\kappa^{*}=\left\{\eta: \delta_{\eta, \kappa} \neq 0\right\}_{\eta} .
$$

Similarly, the transpose $\delta^{T}$ of the matrix $\delta$ induces a map from $C^{1}$ to $C^{0}$, which is defined on $\mathcal{B}^{1}$ as

$$
\begin{aligned}
& \delta^{T}: C^{1} \longrightarrow C^{0} \\
& e_{\alpha}^{1} \longmapsto \delta^{T} e_{\alpha}^{1}=\sum_{i=1}^{n} \delta_{\alpha, i} e_{i}^{0},
\end{aligned}
$$

yields the relation on indices

$$
\begin{aligned}
\{1, \ldots, m\} & \longrightarrow\{1, \ldots, n\} \\
\eta & \longrightarrow \eta^{*}
\end{aligned}
$$

where

$$
\eta^{*}=\left\{\kappa: \delta_{\eta, \kappa} \neq 0\right\}_{\kappa} .
$$

Referring to the chain complex ( $\left.\mathfrak{C}^{\prime \prime \prime}\right)$ described in Sec. IV, we choose bases $\mathcal{B}_{\ell}^{\tau}=\left\{e_{\iota}^{\ell_{\tau}}\right\}$, of $C_{\ell}^{\tau}$ for $\tau=0,1$ and $\ell=$ $A, B, C$. We accordingly fix matrix representations of the maps $\delta_{A}, \delta_{B}$, and $\delta_{C}$; with slight abuse of notation, we indicate with the same symbol the $m_{\ell} \times n_{\ell}$ matrix representation of a map and the map itself. We indicate with $i, j, k$ indices of $\mathcal{B}_{A}^{0}, \mathcal{B}_{B}^{0}$, and $\mathcal{B}_{C}^{0}$, respectively, and with $\alpha, \beta, \gamma$ indices of $\mathcal{B}_{A}^{1}, \mathcal{B}_{A}^{1}, \mathcal{B}_{C}^{1}$. Since we deal with threefold tensor product spaces (e.g., $C_{A}^{0} \otimes C_{B}^{0} \otimes C_{C}^{0}$ ) we consider triplets $(i, j, k)$ of valid indices; we indicate with $\left(i^{*}, j, k\right)$ the set of indices $\left\{(\eta, j, k): \eta \in i^{*}\right\}$, and similarly for any possible triplet combination of starred $\left(\iota^{*}\right)$ and nonstarred ( $)$ indices.

As illustrated in Sec. IV, when defining a CSS code on the chain complex $\left(\mathfrak{C}^{\prime \prime \prime}\right)$, the following relations hold:

1. basis elements of $\mathcal{C}_{0}$ are in one-to-one correspondence with a generating set of $Z$ stabilizers;

2 . basis elements of the vector space $\mathcal{C}_{1}$ are in one-toone correspondence with the qubits;

3 . basis elements of the vector space $\mathcal{C}_{2}$ are in oneto-one correspondence with a generating set of $X$ stabilizers;

4. basis elements of $\mathcal{C}_{3}$ are in one-to-one correspondence with a generating set of metachecks. 
TABLE IV. Notation and correspondences between objects of the chain complex $\left(\mathfrak{C}^{\prime \prime \prime}\right)$.

\begin{tabular}{|c|c|c|}
\hline Object & Indexing & Basis vector \\
\hline \multirow{3}{*}{ Qubits } & $(\alpha, j, k)$ & $\left(e_{\alpha}^{A_{1}} \otimes e_{j}^{B_{0}} \otimes e_{k}^{C_{0}}, \quad 0, \quad 0\right)$ \\
\hline & $(i, \beta, k)$ & $\left(0, \quad e_{i}^{A_{0}} \otimes e_{\beta}^{B_{1}} \otimes e_{k}^{C_{0}}, \quad 0\right)$ \\
\hline & $(i, j, \gamma)$ & $\left(0, \quad 0, \quad e_{i}^{A_{0}} \otimes e_{j}^{B_{0}} \otimes e_{\gamma}^{C_{1}}\right)$ \\
\hline \multirow{3}{*}{$X$ stabilizers } & $(\alpha, \beta, k)$ & $\delta_{2}^{T}\left(e_{\alpha}^{A_{1}} \otimes e_{\beta}^{B_{1}} \otimes e_{k}^{C_{0}}, \quad 0, \quad 0\right)$ \\
\hline & $(\alpha, j, \gamma)$ & $\delta_{2}^{T}\left(0, \quad e_{\alpha}^{A_{1}} \otimes e_{j}^{B_{0}} \otimes e_{\gamma}^{C_{1}}, \quad 0\right)$ \\
\hline & $(i, \beta, \gamma)$ & $\delta_{2}^{T}\left(0, \quad 0, \quad e_{i}^{A_{0}} \otimes e_{\beta}^{B_{1}} \otimes e_{\gamma}^{C_{1}}\right)$ \\
\hline$Z$ stabilizers & $(i, j, k)$ & $\delta_{1}\left(e_{i}^{A_{0}} \otimes e_{j}^{B_{0}} \otimes e_{k}^{C_{0}}\right)$ \\
\hline Metacheck & $(\alpha, \beta, \gamma)$ & $\delta_{3}^{T}\left(e_{\alpha}^{A_{1}} \otimes e_{\beta}^{B_{1}} \otimes e_{\gamma}^{C_{1}}\right)$ \\
\hline
\end{tabular}

Combining these with Eqs. (B2) and (B2 T), we obtain the relations reported in Table IV. More precisely, we choose as bases for the spaces $\mathcal{C}_{3}, \mathcal{C}_{2}, \mathcal{C}_{1}$, and $\mathcal{C}_{0}$ the product bases obtained by combining $\mathcal{B}_{\ell=A, B, C}^{0}$ and $\mathcal{B}_{\ell=A, B, C}^{1}$ and we index qubits, stabilizers, and metachecks on $\mathcal{C}\left(\delta_{A}, \delta_{B}, \delta_{C}\right)$ accordingly. Equivalently, basis vectors are labeled with consecutive integers so as to preserve the ordering induced by the bases.

We use the relations of Table IV to visualize the chain complex $\left(\mathfrak{C}^{\prime \prime \prime}\right)$ on a $3 \mathrm{D}$ cubic lattice. In order to do so, we first fix a coordinate system

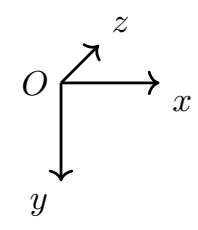

where $O$ is the origin. Since basis vectors are labeled with integers (the $i$ th basis vector corresponds to the integer $i$, and vice versa) we can build a $3 \mathrm{D}$ grid of points where any point corresponds to a basis vector of $\mathcal{C}_{0}, \mathcal{C}_{1}, \mathcal{C}_{2}$, or $\mathcal{C}_{3}$. More precisely we fix a set of valid coordinates for points in the grid:

1. integer coordinates $(z, y, x)=(i, j, k)$ for $i=1, \ldots$, $n_{a}, j=1, \ldots, n_{b}$, and $k=1, \ldots, n_{c}$;

2. half-integers coordinates $(z, y, x)=(\alpha+0.5, \beta+$ $0.5, \gamma+0.5)$ for $\alpha=1, \ldots, m_{a}, \beta=1, \ldots, m_{b}$, and $\gamma=1, \ldots, m_{c}$;

3. the origin has coordinates $O=(1,1,1)$.

In this way, any point with valid coordinates uniquely identifies a basis vector (and therefore an object in the chain complex, see Table IV). For example,
TABLE V. Correspondence between qubits in $\mathcal{C}_{1}$ and edges of the lattice.

\begin{tabular}{lc}
\hline \hline Qubit & \multicolumn{1}{c}{ Edge } \\
\hline $\begin{array}{l}\text { Transverse qubits } \\
(\alpha, j, k)\end{array}$ & Edges parallel to the $z$ axis \\
Vertical qubits & Middle point: $(\alpha+0.5, j, k)$ \\
$(i, \beta, k)$ & Edges parallel to the $y$ axis \\
\hline Horizontal qubits & Middle point: $(i, \beta+0.5, k)$ \\
$(i, j, \gamma)$ & Edges parallel to the $x$ axis \\
\hline \hline
\end{tabular}

1. the point $(1,4,2)$ corresponds to the basis vector $\left(e_{1}^{A_{0}} \otimes e_{4}^{B_{0}} \otimes e_{2}^{C_{0}}\right) \in \mathcal{C}_{0}(Z$ stabilizers $) ;$

2 . the point $(1.5,4,2)$ corresponds to the basis vector $\left(e_{1}^{A_{1}} \otimes e_{4}^{B_{0}} \otimes e_{2}^{C_{0}}, 0,0\right) \in \mathcal{C}_{1}$ (qubits);

3 . the point $(1.5,4,2.5)$ corresponds to the basis vector $\left(0, e_{1}^{A_{1}} \otimes e_{4}^{B_{0}} \otimes e_{2}^{C_{1}}, 0\right) \in \mathcal{C}_{2}(X$ stabilizers $) ;$

4. the point $(1.5,4.5,2.5)$ corresponds to the basis vector $\left(e_{1}^{A_{1}} \otimes e_{4}^{B_{1}} \otimes e_{2}^{C_{1}}\right) \in \mathcal{C}_{3}$ (metachecks).

We draw an edge for any qubit of the code defined on $\left(\mathfrak{C}^{\prime \prime \prime}\right)$. Qubits are in one-to-one correspondence with basis element of $\mathcal{C}_{1}$ and therefore are of three different types: $(v, 0,0),(0, v, 0)$, and $(0,0, v)$. Accordingly, we draw edges of three different types as detailed in Table V (see also Fig. 6). In other words, any point with two integer entries and one half-integer entry is the middle point of an edge of unit length, which corresponds to a qubit. In this way we obtain a cubic lattice with (possibly) some missing edges.

Points with two half-integer and one integer entries do not intersect any edge and sit in the center of a (a)

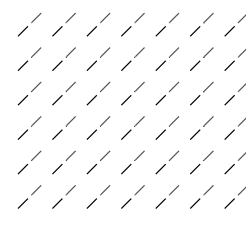

(b)

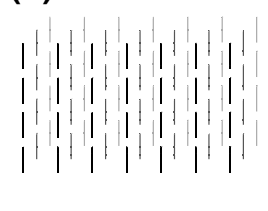

(d)

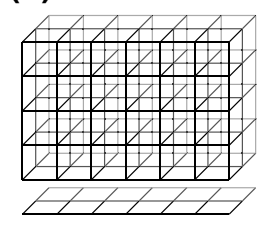

(c)

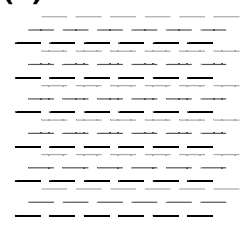

FIG. 6. Graphical representation of the cubic lattice associated to a $3 \mathrm{D}$ product code where the seed matrices $\delta_{A}, \delta_{B}, \delta_{C}$ have size $2 \times 3,4 \times 6$, and $6 \times 7$, respectively. In (a), (b), and (c) only transversal, vertical, and horizontal edges are depicted. In (d) we can see the complete lattice obtained by matching the origin $O=$ $(1,1,1)$ of the three lattices of edges. 
(a)

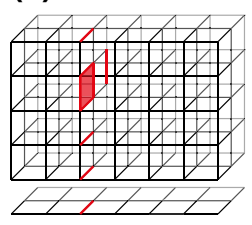

(b)

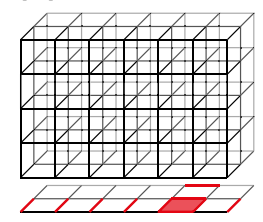

(c)

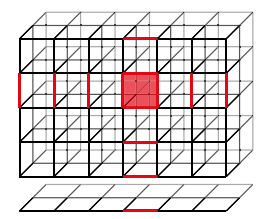

FIG. 7. $X$ stabilizers on the lattice described in Fig. 6. (a) $X$ stabilizer corresponding to the transversal-vertical square indexed by $(\alpha, \beta, k)=(1,2,3)$; its support is contained in the cross of transversal and vertical qubits (red edges) in the $y-z$ plane $\{x=3\}$. The crossing has coordinates $(z, y, x)=$ $(1.5,2.5,3)$ and sits in the center of the red square. (b) $X$ stabilizer corresponding to the transversal-horizontal square indexed by $(\alpha, j, \gamma)=(1,6,5)$; its support is contained in the cross of transversal and vertical qubits (red edges) in the $x-z$ plane $\{y=$ $6\}$. The crossing has coordinates $(z, y, x)=(1.5,6,5.5)$ and sits in the center of the red square. (c) $X$ stabilizer corresponding to the vertical-horizontal square indexed by $(i, \beta, \gamma)=(1,4,2)$; its support is contained in the cross of transversal and vertical qubits (red edges) in the $x-y$ plane $\{z=1\}$. The crossing has coordinates $(z, y, x)=(1,2.5,4.5)$ and sits in the center of the red square.

(possibly incomplete) square face. These points correspond to $X$ stabilizers, which we therefore identify with faces. Given a triplet corresponding to one of such a point, the associated $X$ stabilizer has support contained in the set of edges, which are parallel to the edges of the square, forming a cross in a plane. $X$ stabilizers, like qubits, are of three different types, being in one-toone correspondence with basis elements of $\mathcal{C}_{2}$. Namely, each $X$ stabilizer in $\mathcal{C}_{2}$ has support in two out of three types of qubits: transverse-vertical, transverse-horizontal, or vertical-horizontal (see Table VI and Fig. 7).

Points with integer coordinates are associated to $Z$ stabilizers; these are points where endpoints of edges intersect.

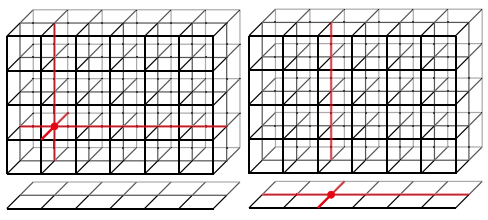

FIG. 8. $Z$ stabilizers on the lattice described in Fig. 6. (a) $Z$ stabilizer corresponding to the vertex indexed by $(i, j, k)=(2,4,2)$; its support is contained in the cross of qubits highlighted as red edges in the picture. The crossing has coordinates $(z, y, x)=$ $(2,4,2)$ (red circle). (b) $Z$ stabilizer corresponding to the vertex indexed by $(i, j, k)=(3,6,2)$; its support is contained in the cross of qubits highlighted as red edges in the picture. The crossing has coordinates $(z, y, x)=(3,6,2)$ (red circle).

The $Z$ stabilizer corresponding to $(i, j, k)$ has support on a $3 \mathrm{D}$ cross of edges and qubits centered in $(z, y, x)=(i, j, k)$ (see Table VI and Fig. 8).

Points with half-integer coordinates sit in the center of a cube. To any such cube is associated a metacheck in $\mathcal{C}_{3}$. Metachecks have support on a 3D cross of faces and $X$ stabilizers parallel to the faces of the cube they are associated to (see Table VI).

\section{On geometric locality}

One interesting feature of the embedding of $3 \mathrm{D}$ product codes on a cubic lattice is that it preserves some locality properties of the seed matrices $\delta_{A}, \delta_{B}$, and $\delta_{C}$. Thus, if we were able to place qubits on a $3 \mathrm{D}$ cubic lattice we could use the 3D homological product to build LDPC codes with nearest-neighbor interactions.

Let $\delta$ be an $m \times n$ matrix with row and column indices $\alpha \in\{1, \ldots, m\}$ and $i \in\{1, \ldots, n\}$, respectively, and let $v=$ $\max \{m, n\}$. We say that $\delta$ is geometrically $\rho$ local on a

TABLE VI. Correspondence between operators of the chain complex $\left(\mathfrak{C}^{\prime \prime \prime}\right)$, their type as geometric objects on the lattice, and their support. Note that the support of $X$ and $Z$ stabilizers is a set of qubits and edges while the support of metachecks is a set of $X$ stabilizers and faces.

\begin{tabular}{|c|c|c|}
\hline Operator & Type & Support \\
\hline$X$ stabilizers & $\begin{array}{c}\text { Transverse-vertical square } \\
(\alpha, \beta, k) \\
\text { Transverse-horizontal square } \\
(\alpha, j, \gamma) \\
\text { Vertical-horizontal square } \\
(i, \beta, \gamma)\end{array}$ & $\begin{array}{l}\text { Transverse qubits: }\left(\alpha, \beta^{*}, k\right) \\
\text { Vertical qubits: }\left(\alpha^{*}, \beta, k\right) \\
\text { Transverse qubits: }\left(\alpha, j, \gamma^{*}\right) \\
\text { Horizontal qubits: }\left(\alpha^{*}, j, \gamma\right) \\
\text { Vertical qubits: }\left(i, \beta, \gamma^{*}\right) \\
\text { Horizontal qubits: }\left(i, \beta^{*}, \gamma\right)\end{array}$ \\
\hline$Z$ stabilizers & $(i, j, k)$ & $\begin{array}{l}\text { Transverse qubits: }\left(i^{*}, j, k\right) \\
\text { Vertical qubits: }\left(i, j^{*}, k\right) \\
\text { Horizontal qubits: }\left(i, j, k^{*}\right)\end{array}$ \\
\hline Metachecks & $(\alpha, \beta, \gamma)$ & $\begin{array}{c}\text { Transverse-vertical faces: }\left(\alpha, \beta, \gamma^{*}\right) \\
\text { Transverse-horizontal faces: }\left(\alpha, \beta^{*}, \gamma\right) \\
\text { Vertical-horizontal faces: }\left(\alpha^{*}, \beta, \gamma\right)\end{array}$ \\
\hline
\end{tabular}


torus if for any row and any column index

$$
\alpha^{*} \subseteq U_{\rho, v}(\alpha) \text { and } i^{*} \subseteq U_{\rho, v}(i),
$$

where $U_{\rho, v}(\zeta)$ is any set of $\rho$ consecutive integers modulo $v$, which contains $\zeta$. In particular, we require the $\alpha$ th rows to have support on columns with index that is close to the integer $\alpha$, and similar for columns. The reason for this choice will be clear when we prove Proposition 1. Briefly, conditions (B3) say that $\delta$ is geometrically $\rho$ local on a torus if the following hold: (1) any of its rows has support on a bounded box of $\rho$ columns, and the box for row $\alpha+1$ is a right shift of the box for row $\alpha$; (2) any of its columns has support on a bounded box of $\rho$ rows, and the box for column $i+1$ is a downward shift of the box for column $i$. In particular, if we associate row and column indices with integer points on a circle of $v$ points:

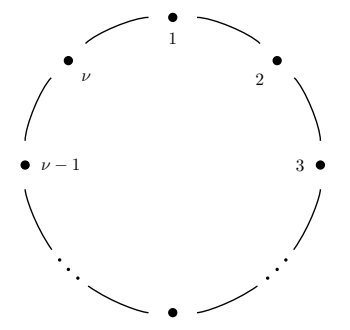

locality means that any set $\alpha^{*} / i^{*}$ is contained in a closed interval on the circle such that (i) it has length at most $\rho$ and (ii) it contains the point $\alpha / i$. For instance, the degenerate parity-check matrix of the repetition code:

$$
\left(\begin{array}{lllll}
1 & 1 & 0 & 0 & 0 \\
0 & 1 & 1 & 0 & 0 \\
0 & 0 & 1 & 1 & 0 \\
0 & 0 & 0 & 1 & 1 \\
1 & 0 & 0 & 0 & 1
\end{array}\right)
$$

is $\rho$ local for $\rho=2$.

A closely related notion of locality on a torus is geometric locality in Euclidean space. We say that an $m \times n$ matrix is geometrically $\rho$ local in Euclidean space if for any row and column index

$$
\alpha^{*} \subseteq U_{\rho}(\alpha) \text { and } i^{*} \subseteq U_{\rho}(i),
$$

where $U_{\rho}(\zeta)$ is any set of $\rho$ consecutive integer in $[1, \ldots, v], v=\max \{m, n\}$, which contains $\zeta$. In this case we can graphically picture locality by associating row and column indices with integer points on a line of $v$ points:

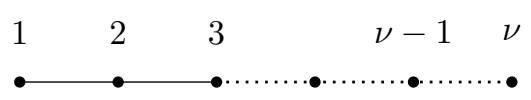

A matrix is local if any set $\alpha^{*} / i^{*}$ is contained in a closed interval on the line such that (i) it has length at most $\rho$ and (ii) it contains the point $\alpha / i$. For example, the full-rank parity-check matrix of the repetition code:

$$
\left(\begin{array}{lllll}
1 & 1 & 0 & 0 & 0 \\
0 & 1 & 1 & 0 & 0 \\
0 & 0 & 1 & 1 & 0
\end{array}\right)
$$

is 2 local.

Geometric locality also applies to codes other than the repetition code. For instance, the matrix

$$
H=\left(\begin{array}{lllllllll}
1 & 1 & 0 & 0 & 0 & 0 & 0 & 0 & 0 \\
0 & 1 & 0 & 0 & 0 & 0 & 1 & 0 & 0 \\
0 & 0 & 1 & 1 & 0 & 0 & 0 & 0 & 0 \\
0 & 0 & 0 & 1 & 0 & 0 & 0 & 1 & 0 \\
0 & 0 & 0 & 0 & 1 & 1 & 0 & 0 & 0 \\
0 & 0 & 0 & 0 & 0 & 1 & 0 & 0 & 1 \\
1 & 0 & 1 & 0 & 1 & 0 & 0 & 0 & 0
\end{array}\right)
$$

obtained via the edge augmentation procedure presented in Ref. [56] is 7 local on a torus. We remark that geometric locality is a property of matrices. For example, the matrix with the same row as $H$ but different ordering $\{1,2,3,4,7,5,6\}$, is geometrically 5 local on a torus.

In general, geometric locality is a relaxation of the locality property of the repetition code, which only allows for interactions between pairs of nearest bits. Importantly, as Proposition 1 states, it is preserved by the 3D product construction. For this reason, geometrically local classical codes, combined with the $3 \mathrm{D}$ product construction, could be good candidates in the quest to quantum local codes beyond the toric and the surface codes.

The remainder of this appendix is organized as follows. We first state Proposition 1 and prove that geometric locality is preserved by the $3 \mathrm{D}$ product construction. We conclude by observing how this proof provides an explicit identification of the 3D toric and surface codes as 3D product codes.

To ease the notation, in the following we shortly refer to codes as geometrically local, dropping the specification on a torus or in Euclidean space. When considering qubits on a cubic lattice, the lattice would be on a torus or in Euclidean space depending on the definition of locality that applies to the seed matrices.

Proposition 1. Consider the $3 D$ product code obtained from three seed matrices geometrically $\rho$ local. If its qubits are displayed on the edges of a cubic lattice as detailed in Appendix B, then it is geometrically $\rho$ local in the following sense:

1. any $X$-stabilizer generator has weight at most $2 \rho$ with support contained in a $2 D$ box of size $\rho \times \rho$;

2. any Z-stabilizer generator has weight at most $3 \rho$ with support contained in a $3 D$ box of size $\rho \times \rho \times \rho$. 
We prove the condition on the $Z$ stabilizers, the proof for the $X$ stabilizer being similar.

Let $S_{z}$ be a $Z$-stabilizer generator. As reported in Tables IV and VI, it is the image of a basis vector $\left(e_{i}^{A_{0}} \otimes e_{j}^{B_{0}} \otimes e_{k}^{C_{0}}\right) \in \mathcal{C}_{0}$ via the map $\delta_{1}$ and it corresponds to the point on the lattice of integers coordinates $(i, j, k)$. By exploiting the choice of the basis for the spaces $\mathcal{C}_{0}$ and $\mathcal{C}_{1}$ and some linear algebra:

$$
\begin{aligned}
\delta_{1}\left(e_{i}^{A_{0}} \otimes e_{j}^{B_{0}} \otimes e_{k}^{C_{0}}\right) & =\sum_{\alpha \in i^{*}}\left(e_{\alpha}^{A_{1}} \otimes e_{j}^{B_{0}} \otimes e_{k}^{C_{0}}, 0,0\right) \\
& +\sum_{\beta \in j^{*}}\left(0, e_{i}^{A_{0}} \otimes e_{\beta}^{B_{1}} \otimes e_{k}^{C_{0}}, 0\right) \\
& +\sum_{\gamma \in k^{*}}\left(0,0, e_{i}^{A_{0}} \otimes e_{j}^{B_{0}} \otimes e_{\gamma}^{C_{1}}\right) .
\end{aligned}
$$

Again using Table IV, the set of indices, which corresponds to this sum of basis vectors of $\mathcal{C}_{1}$, can be written as

$$
\begin{aligned}
\operatorname{indices}\left(S_{z}\right)= & \left\{(\alpha, j, k): \alpha \in i^{*}\right\} \\
& \cup\left\{(i, \beta, k): \beta \in j^{*}\right\} \\
& \cup\left\{(i, j, \gamma): \gamma \in k^{*}\right\} .
\end{aligned}
$$

Following the nomenclature of qubits as traversal, vertical, and horizontal, we see that the three components of the support of $S_{z}$ given above respect this division and therefore we can write

$$
\operatorname{indices}\left(S_{z}\right)=\operatorname{indices}\left(S_{z}\right)_{t} \cup \operatorname{indices}\left(S_{z}\right)_{v} \cup \operatorname{indices}\left(S_{z}\right)_{h} \text {. }
$$

Using (B4) [or (B3)], we see that the sets indices $\left(S_{z}\right)_{t}$, indices $\left(S_{z}\right)_{v}$, and indices $\left(S_{z}\right)_{h}$ correspond, respectively, to the three sets of consecutive coordinates on the lattice:

$$
\begin{aligned}
\Pi_{t} & =\left\{(\bar{i}, j, k): \bar{i} \in U_{\rho}(i)\right\}, \\
\Pi_{v} & =\left\{(i, \bar{j}, k): \bar{j} \in U_{\rho}(j)\right\}, \\
\Pi_{h} & =\left\{(i, j, \bar{k}): \bar{k} \in U_{\rho}(k)\right\} .
\end{aligned}
$$

Since we require $\zeta \in U_{\rho}(\zeta)$ [or $\zeta \in U_{\rho, v}(\zeta)$ ], the three sets of coordinates intersect on the point $(z, y, x)=(i, j, k)$. Moreover, all three intervals $\Pi_{t}, \Pi_{v}, \Pi_{h}$ have length at most $\rho$. Combining these, we find that the support of $S_{z}$ indexed by $(i, j, k)$ is contained in in a $\rho \times \rho \times \rho$ neighborhood of the point $(z, y, x)=(i, j, k)$ and has cardinality at most $3 \rho$. In other words, we show that the support of $S_{z}$ is contained on a 3D cross of qubits with arms of length at most $\rho$.

As previously said, the $3 \mathrm{D}$ toric and planar codes are particular instances of the 3D product construction. Furthermore, it is well known that they are local on a torus and in the Euclidean space, respectively. To see how this is the case, we remind the reader that the $3 \mathrm{D}$ toric code is obtained by choosing as seed matrices the degenerate parity-check matrix of the repetition code in the standard basis. For matrix size $L \times L$, it holds that

$$
\begin{aligned}
\{1, \ldots, L\} & \longleftrightarrow\{1, \ldots, L\} \\
i & \longrightarrow\{i, i+1 \bmod L\} \\
\{\alpha, \alpha+1 \bmod L\} & \longleftrightarrow \alpha .
\end{aligned}
$$

Therefore, stabilizers have support on pairs of consecutive edges, and it is straightforward to see that they have the usual shape:

1. $Z$ stabilizers have support on edges incident to a vertex;

2. $X$ stabilizers have support on edges on the boundary of a square face;

3. metachecks have support on the faces of a cube.

A similar argument holds for the 3D surface code, which is local in Euclidean space.

\section{APPENDIX C: ALL 3D PRODUCT CODES HAVE X-CONFINEMENT}

In this section we prove Theorem 1, which states that all 3D product codes have $X$ confinement. Our proof follows the proof of soundness for 4D codes given in Ref. [18] with some minor adaptions and it is here reported for completeness.

First, we show that an opportunely chosen length-2 chain complex has confined maps. Secondly, we explain how to use this chain complex as a building block of the length-3 chain complex ( $\left.\mathfrak{C}^{\prime \prime \prime}\right)$ described in Sec. IV. Lastly, we prove that the confinement property is preserved and thus $3 \mathrm{D}$ codes defined on $\left(\mathfrak{C}^{\prime \prime \prime}\right)$ as explained in Sec. IV have $X$ confinement.

Let $\delta_{A}: C_{A}^{0} \rightarrow C_{A}^{1}$ and $\delta_{B}: C_{B}^{0} \rightarrow C_{B}^{1}$ be two length1 chain complexes. We consider the length-2 product complex $\tilde{\mathfrak{C}}$ defined as

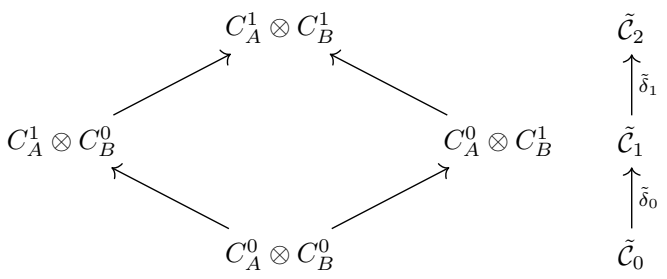

where

$$
\begin{aligned}
& \tilde{\delta}_{0}=\left(\begin{array}{ll}
\delta_{A} \otimes \mathbb{1} & 1 \otimes \delta_{B}
\end{array}\right), \\
& \tilde{\delta}_{1}=\left(\begin{array}{l}
\mathbb{1} \otimes \delta_{B} \\
\delta_{A} \otimes \mathbb{1}
\end{array}\right) .
\end{aligned}
$$

We first show that the map $\tilde{\delta}_{0}$ has confinement. 
Lemma 9. $\tilde{\delta}_{0}$ has $(t, f)$ confinement where $t=\min \left\{d_{A}, d_{B}\right\}$ and $f(x)=x^{2} / 4$.

In order to prove Lemma 9 we first introduce some useful notation. When considering vectors $v$ in a twofold tensor product space $\mathbb{F}^{n_{1}} \otimes \mathbb{F}^{n_{2}}$ it can be handy to consider their reshaping, which is a $n_{1} \times n_{2}$ matrix on $\mathbb{F}$. Namely, fixed bases $\mathcal{B}^{1}=\left\{a_{1}, \ldots, a_{n_{1}}\right\}$ and $\mathcal{B}^{2}=\left\{b_{1}, \ldots, b_{n_{2}}\right\}$ of $\mathbb{F}^{n_{1}}$ and $\mathbb{F}^{n_{2}}$, respectively, their product

$$
\mathcal{B}=\left\{a_{i} \otimes b_{j}\right\}_{\substack{i=1, \ldots, n_{1} \\ j=1, \ldots, n_{2}}}
$$

is a basis of $\mathbb{F}^{n_{1}} \otimes \mathbb{F}^{n_{2}}$. Therefore, we can write

$$
v=\sum_{a_{i} \otimes b_{j} \in \mathcal{B}} v_{i j} a_{i} \otimes b_{j}
$$

for some $v_{i j} \in \mathbb{F}$. We call the matrix $V$ whose entries are the coefficient $v_{i j}$ the reshaping of $v$. Given matrices $M$ and $N$ of size $n_{1} \times m_{1}$ and $n_{2} \times m_{2}$ associated to linear maps from $\mathbb{F}^{n_{1}}$ and $\mathbb{F}^{n_{2}}$, respectively, the map $M \otimes N$ from $\mathbb{F}^{n_{1}} \otimes \mathbb{F}^{n_{2}}$ to $\mathbb{F}^{m_{1}} \otimes \mathbb{F}^{m_{2}}$ acts on the reshaping of $v$ as

$$
(M \otimes N) V \longmapsto M V N^{T} .
$$

In the following we always indicate with lowercase symbol vectors and with the corresponding uppercase symbols their reshaping. We can now use this notation to prove Lemma 9. Let $v \in C_{0}^{A} \otimes C_{0}^{B}$ and let $s=\tilde{\delta}_{0}(v)$. By reshaping,

$$
S=\left(\begin{array}{c}
\delta_{A} V \\
V \delta_{B}^{T}
\end{array}\right)
$$

If we assume $|v|=|v|^{\text {red }} \leq t=\min \left\{d_{A}, d_{B}\right\}$ then $V$ has no column in $\operatorname{ker} \delta_{A}$ and no row in $\operatorname{ker} \delta_{B}^{T}$ so that

$$
\operatorname{col}\left(\delta_{A} V\right)=\operatorname{col}(V) \text { and } \operatorname{row}\left(V \delta_{B}^{T}\right)=\operatorname{row}(V),
$$

where $\operatorname{col}(V) / \operatorname{row}(V)$ is the number of nonzero columns and rows of the matrix $V$. Therefore, for the weight of $S$, it holds that

$$
\begin{aligned}
|S|=\left|\delta_{A} V\right|+\left|V \delta_{B}^{T}\right| & \geq \operatorname{col}\left(\delta_{A} V\right)+\operatorname{row}\left(V \delta_{B}^{T}\right) \\
& =\operatorname{col}(V)+\operatorname{row}(V) .
\end{aligned}
$$

Combining this with $(a+b)^{2} / 4 \geq a b$ for integers $a, b$ yields

$$
|S|^{2} / 4 \geq \operatorname{col}(V) \cdot \operatorname{row}(V) \geq|V| .
$$

Corollary 1 below follows easily from Lemma 9 .

Corollary 1. If $\delta_{A}$ or $\delta_{B}$ have $(g, t)$ confinement with $g$ increasing and subadditive [69] then $\tilde{\delta}_{0}$ has $(g, t)$ confinement too.
Without loss of generality, we assume that $\delta_{A}$ has $(t, g)$ confinement (the proof for $\delta_{B}$ being symmetrical).

Consider the syndrome matrix

$$
S=\left(\begin{array}{l}
\delta_{A} V \\
V \delta_{B}^{T}
\end{array}\right)=\left(\begin{array}{l}
S_{1} \\
S_{2}
\end{array}\right)
$$

where $V$ is the reshaping of a vector $v$ of reduced weight less than $t$, i.e., $|v|=|v|^{\text {red }} \leq t$. Because $\delta_{A}$ has confinement, for the column of $V$ it holds that

$$
\left|S_{1}^{j}\right| \geq g\left(\left|V^{j}\right|\right)
$$

Thus, we can use confinement columnwise and obtain

$$
\begin{aligned}
& \left|S_{1}\right|=\sum_{j}\left|S_{1}^{j}\right| \quad \text { by definition of }|\cdot| \\
& \geq \sum_{j} g\left(\left|V^{j}\right|\right) \quad \text { by confinement of } \delta_{A} \\
& \geq g\left(\sum_{j}\left|V^{j}\right|\right) \quad \text { by subadditivity of } g \\
& \geq g(|V|) \quad \text { by definition of }|\cdot| \text {. }
\end{aligned}
$$

Combining this and

$$
|S|=\left|S_{1}\right|+\left|S_{2}\right| \geq\left|S_{1}\right|
$$

yields $|S| \geq g(|V|)$.

Loosely, Corollary 1 states that the result of Lemma 9 can be improved whenever at least one of the seed matrices $\delta_{A}$ and $\delta_{B}$ used to build the length-2 product complex $(\tilde{\mathfrak{C}})$ shows linear confinement. However, this is not sufficient to prove that the quantum code $\mathcal{C}\left(\mathfrak{C}_{1}\right)$ associated to $(\tilde{\mathfrak{C}})$ by equating $H_{X}=\tilde{\delta}_{1}, H_{Z}=\tilde{\delta}_{0}^{T}$ has confinement. In fact, here we prove that the matrix $H_{Z}^{T}$ has confinement and not that one of the syndrome matrices $H_{Z}$ or $H_{X}$ have it. In other words, Corollary 1 it is not sufficient to infer the construction of expander codes outlined in Ref. [20]; here confinement goes in the "wrong" direction, namely as the transpose of the syndrome map. Even if not interestingly on its own, Corollary 1 can be used to improve the confinement function of the $X$-syndrome map of the code $\mathcal{C}\left(\delta_{A}, \delta_{B}, \delta_{C}\right)$.

More generally, we want to use Lemma 9 to infer that the code $\mathcal{C}\left(\delta_{A}, \delta_{B}, \delta_{C}\right)$ defined on the chain complex $\left(\mathfrak{C}^{\prime \prime \prime}\right)$ has $X$ confinement. To see how this is the case, we consider an "asymmetrical" version of $\left(\mathfrak{C}^{\prime \prime \prime}\right)$ as the product of the length-2 chain complex $(\tilde{\mathfrak{C}})$ and the length-1 chain complex $\delta_{C}: C_{0}^{C} \rightarrow C_{1}^{C}$. The asymmetric product complex $\breve{\mathfrak{C}}$ is then 


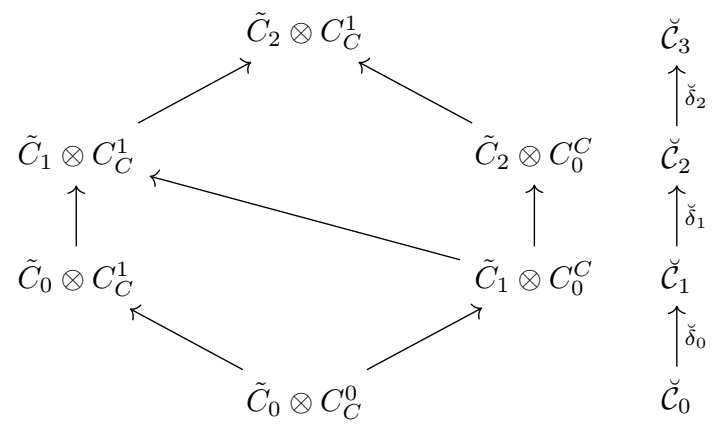

$(\breve{\mathfrak{C}})$

where

$$
\begin{aligned}
& \breve{\delta}_{0}=\left(\begin{array}{c}
\mathbb{1} \otimes \delta_{C} \\
\tilde{\delta}_{0} \otimes \mathbb{1}
\end{array}\right), \\
& \breve{\delta}_{1}=\left(\begin{array}{cc}
\tilde{\delta}_{0} \otimes \mathbb{1} & \mathbb{1} \otimes \delta_{C} \\
0 & \tilde{\delta}_{1} \otimes \mathbb{1}
\end{array}\right), \\
& \breve{\delta}_{2}=\left(\begin{array}{ll}
\tilde{\delta}_{1} \otimes \mathbb{1} & \mathbb{1} \otimes \delta_{C}
\end{array}\right) .
\end{aligned}
$$

Claim 1. Let $(v, w) \in \breve{\mathcal{C}}_{1}$ have weight less than $t$ and $s=$ $\breve{\delta}_{1}(v, w)$ be its syndrome. If $(V, W)$ is the reshaping of the vector $(v, w)$ then the following syndrome equation holds:

$$
S=\left(\begin{array}{c}
S_{1} \\
S_{2}
\end{array}\right)=\left(\begin{array}{c}
\tilde{\delta}_{0} V+W \delta_{C}^{T} \\
\tilde{\delta}_{1} W
\end{array}\right)
$$

where $S$ is the reshaping of $s$.

Note that a stabilizer for the chain complex $\breve{\mathcal{C}}_{0} \rightarrow \breve{\mathcal{C}}_{1} \rightarrow$ $\breve{\mathcal{C}}_{2} \rightarrow \breve{\mathcal{C}}_{3}$ and the syndrome map $\breve{\delta}_{1}(\cdot)$ has the form $\breve{\delta}_{0}(\mathrm{~m})$ for some $m \in \breve{\mathcal{C}}_{0}$. By construction, we can add any stabilizer to $(v, w)$ without violating the syndrome Eq. (SE). In particular,

1. $|(v, w)|<t$ entails that its reshaping satisfies the following properties:

(a) Both V and W have at most t nonzero rows. Thus all their columns have weight at most $t$.

(b) Both $V$ and $W$ has at most $t$ nonzero columns. Thus all their rows have weight at most $t$.

2. Fix a row index $i$ and a column index $j$. Let $M$ be a matrix in $\breve{\mathcal{C}}_{0}$ with columns

$$
M^{h}= \begin{cases}\nu^{j} & \text { for hin } \operatorname{supp}\left(W \delta_{C}^{T}\right)_{i}=\operatorname{supp}\left(W_{i} \delta_{C}^{T}\right) \\ 0 & \text { elsewhere }\end{cases}
$$

Its image $\left(M \delta_{C}^{T}, \tilde{\delta}_{0} M\right)$ through $\breve{\delta}_{0}$ is a stabilizer. Define $V^{*}$ and $W^{*}$ as

$$
V^{*}=V+M \delta_{C}^{T} \text { and } W^{*}=W+\tilde{\delta}_{0} M .
$$

Observe that (a) $M$ is a matrix whose nonzero columns are equal to a column of $V$. Therefore, $M$ has row support contained in the row support of $V$ :

$$
\operatorname{row}\left(V^{*}\right) \subseteq \operatorname{row}(V) .
$$

(b) $M$ is a matrix whose column support is $\operatorname{supp}\left(W_{i} \delta_{C}^{T}\right)$ for some row $W_{i}$ of $W$. Therefore, $M$ has column support contained in the column support of $W$ :

$$
\operatorname{col}\left(W^{*}\right) \subseteq \operatorname{col}(W) .
$$

Lemma 10 (Inheritance of confinement). $\breve{\delta}_{1}$ has $(t, f)$ confinement, where $t=\min \left\{d_{A}, d_{B}, d_{C}\right\}$ and $f(x)=x^{3} / 4$.

Let $(v, w) \in \breve{\mathcal{C}_{1}}$ be such that $|(v, w)|=|(v, w)|^{\text {red }} \leq t$ and $s=\breve{\delta}_{1}(v, w)$ be its syndrome. Reshaping vectors into matrices [see Eqs. (C1) and (C2)] yields the following syndrome equation:

$$
S=\left(\begin{array}{c}
S_{1} \\
S_{2}
\end{array}\right)=\left(\begin{array}{c}
\tilde{\delta}_{0} V+W \delta_{C}^{T} \\
\tilde{\delta}_{1} W
\end{array}\right)
$$

We transform the vector $(V, W)$ by adding stabilizers to it in order to change its column and row support. We do this by iterating the following two steps.

Step 1: Let $i, j$ be row and column indices such that

(a) $\left(W \delta_{C}^{T}\right)_{i} \neq 0$ and $\left(S_{1}\right)_{i}=0$;

(b) $\left(\tilde{\delta}_{0} V\right)_{i j} \neq 0$ and $\left(W \delta_{C}^{T}\right)_{i j}=1$.

Build a matrix $M$ as in Claim 1 .

Transform $V$ and $W$ accordingly:

$$
\begin{gathered}
V \longmapsto V+M \delta_{C}^{T}, \\
W \longmapsto W+\tilde{\delta}_{0} M .
\end{gathered}
$$

Note that in this way we are able to delete row $i$ of $W \delta_{C}^{T}$.

Iterate this step until we obtain

$$
\operatorname{row}\left(W \delta_{C}^{T}\right) \subseteq \operatorname{row}\left(S_{1}\right)
$$

Step 2: Let $i, j$ be row and column indices such that

(a) $\left(W \delta_{C}^{T}\right)^{j} \neq 0$ and $\left(S_{1}\right)^{j}=0$; this entails $\left(\tilde{\delta}_{0} V\right)^{j}=$ $\left(W \delta_{C}^{T}\right)^{j}$

(b) $\left(\tilde{\delta}_{0} V\right)_{i j}=\left(W \delta_{C}^{T}\right)_{i j}=1$.

Build a matrix $M$ as in Claim 1 .

Transform $V$ and $W$ accordingly:

$$
\begin{gathered}
V \longmapsto V+M \delta_{C}^{T}, \\
W \longmapsto W+\tilde{\delta}_{0} M .
\end{gathered}
$$

Note that in this way we are able to delete row $i$ of $W \delta_{C}^{T}$ and by repeatedly doing so we can delete any column $j$ 
of $W\left(\delta_{C}^{T}\right)$, which does not belong to the column support of $S_{1}$.Iterate this step until we obtain

$$
\operatorname{col}\left(W \delta_{C}^{T}\right) \subseteq \operatorname{col}\left(S_{1}\right)
$$

Let $\mathbf{M}$ be the matrix formed by summing over all the matrices $M$ found during these two steps. Define $V^{*}$ and $W^{*}$ as

$$
V^{*}=V+\mathbf{M} \delta_{C}^{T} \text { and } W^{*}=W+\tilde{\delta}_{0} \mathbf{M} .
$$

We now proceed to prove an upper bound for the weight of $W^{*}$ first and then one for the weight of $V^{*}$. By combining these two bounds we obtain the desired confinement relation between the weight of the syndrome and the weight of the error.

\section{BOUND ON THE WEIGHT OF $W^{*}$}

1. By Claim 1, no row of $W^{*}$ has weight more than $t$ and therefore none of them belongs to $\operatorname{ker} \delta_{C}^{T}$ so that $\operatorname{row}\left(W^{*} \delta_{C}^{T}\right)=\operatorname{row}\left(W^{*}\right)$. Combining this with Eq. (C3) yields

$$
\operatorname{row}\left(W^{*}\right) \subseteq \operatorname{row}\left(S_{1}\right)
$$

2. By Claim 1, the column support of $W^{*}$ is contained in the column support of $W$, which is equal to the column support of $S_{2}$, by assumption on its weight. Summing these up,

$$
\operatorname{col}\left(W^{*}\right) \subseteq \operatorname{col}\left(S_{2}\right)
$$

3. Combining Eqs. (C5) and (C6) yields

$$
\left|S_{1}\right|\left|S_{2}\right| \geq\left|W^{*}\right|
$$

\section{BOUND ON THE WEIGHT OF $V^{*}$.}

1. By rearranging the syndrome Eq. (SE), we can write $\tilde{\delta}_{0} V^{*}=S_{1}+W^{*} \delta_{C}^{T}$. Equations (C3) and (C4) therefore entail

$$
\operatorname{row}\left(\tilde{\delta}_{0} V^{*}\right) \subseteq \operatorname{row}\left(S_{1}\right)
$$

and

$$
\operatorname{col}\left(\tilde{\delta}_{0} V^{*}\right) \subseteq \operatorname{col}\left(S_{1}\right)
$$

2. By Claim 1, the row support of $V^{*}$ is contained in the row support of $V$, which has cardinality at most $t$. In particular, all the columns of $V^{*}$ have weight at most $t$ and therefore we can use the confinement property of the map $\tilde{\delta}_{0}$ columnwise (see Lemma 9). In other words, for each column $j$ of $V^{*}$, the following holds:

$$
\frac{\left|\left(\tilde{\delta}_{0} V^{*}\right)^{j}\right|^{2}}{4} \geq\left|\left(V^{*}\right)^{j}\right|
$$

Combining this with Eq. (C7) yields

$$
\frac{\left|\operatorname{row}\left(S_{1}\right)\right|^{2}}{4} \geq\left|\left(V^{*}\right)^{j}\right|
$$

3. By Claim 1, no column of $V^{*}$ has weight more than $t$ and therefore none of them belongs to $\operatorname{ker} \tilde{\delta}_{0}$ so that $\operatorname{col}\left(V^{*}\right)=\operatorname{col}\left(\tilde{\delta}_{0} V^{*}\right)$. By Eq. (C9) this entails

$$
\operatorname{col}\left(V^{*}\right) \subseteq \operatorname{col}\left(S_{1}\right)
$$

In other words, $V^{*}$ has at most $\left|\operatorname{col}\left(S_{1}\right)\right|$ nonzero columns and combining this with Eq. (SE) yields

$$
\frac{\left|\operatorname{row}\left(S_{1}\right)\right|^{2}}{4}\left|\operatorname{col}\left(S_{1}\right)\right| \geq\left|V^{*}\right|
$$

which entails

$$
\frac{1}{4}\left|S_{1}\right|^{3} \geq\left|V^{*}\right| .
$$

Since $|S|=\left|S_{1}\right|+\left|S_{2}\right|$ and $|(V, W)|=|V|+|W|$, we can add the bounds found for $V^{*}$ and $W^{*}$. Observing that $(a+b)^{3} \geq\left(a^{3}+a^{2} b+a b\right)$ for integer $a, b$, we obtain that $\left(v^{*}, w^{*}\right)$ is a vector equivalent to $(v, w)$ [i.e., it satisfies the syndrome Eq. (SE)] for which it holds

$$
\frac{1}{4}|s|^{3} \geq\left|\left(v^{*}, w^{*}\right)\right| .
$$

In conclusion, since $\left|\left(v^{*}, w^{*}\right)\right| \geq|(v, w)|=|(v, w)|^{\text {red }}$, we prove that $\breve{\delta}_{1}$ has confinement with respect to the function $f(x)=x^{3} / 4$.

It is sometimes possible to find a better confinement function $f$ for the map $\breve{\delta}_{1}$ when $\tilde{\delta}_{0}$ has $(t, g)$ confinement, for instance, as per Corollary 1. In fact, in such a case, Eq. (C9) becomes

$$
g\left(\left|\tilde{\delta}_{0} V^{* j}\right|\right) \geq\left|\left(V^{*}\right)^{j}\right|,
$$

and combining this with Eq. (C7) yields

$$
g\left(\operatorname{row}\left(S_{1}\right)\right) \geq\left|\left(V^{*}\right)^{j}\right| .
$$

Thanks to Eq. (C10) we obtain

$$
g\left(\operatorname{row}\left(S_{1}\right)\right)\left|\operatorname{col}\left(S_{1}\right)\right| \geq\left|V^{*}\right|,
$$

which, for $g$ increasing, entails

$$
g\left(\left|S_{1}\right|\right)\left|S_{1}\right| \geq\left|V^{*}\right| .
$$


Summing up,

$$
\left|S_{1}\right|\left|S_{2}\right|+g\left(\left|S_{1}\right|\right)\left|S_{1}\right| \geq\left|V^{*}\right|+\left|W^{*}\right| .
$$

In other words, depending on the confinement function $g$ for $\tilde{\delta}_{0}$, Eq. (C12) can be used to find better confinement function $f$ for $\breve{\delta}_{1}$. For instance, if $g(x)=\alpha x$ is linear then $f(x)=\hat{\alpha} x^{2}$, for $\hat{\alpha}=\max \{\alpha, 1\}$ is a confinement function for $\breve{\delta}_{1}$. To sum up, whenever at least one of the seed matrices $\delta_{A}, \delta_{B}, \delta_{C}$ has linear confinement, the associated $3 \mathrm{D}$ product code has quadratic confinement.

We do not rule out the existence of a direct relationship between the confinement function for the seed matrices $\delta_{A}, \delta_{B}$, and $\delta_{C}$ and the confinement function for the corresponding $\breve{\delta}_{1}$ map of their $3 \mathrm{D}$ product code. In fact, we do believe that the cubic factor of Lemma 10 is an artefact of our proof and not a tight bound. For instance, when considering the $3 \mathrm{D}$ toric or surface code we find a quadratic relationship between the error size and syndrome size that follows a surface area-perimeter law.

\section{APPENDIX D: ON SOME PROPERTIES OF EXAPANDER CODES}

Here we prove that the family of expander codes considered in Ref. [20] has the three properties stated in the main text, namely,

(i) they have full-rank parity-check matrices;

(ii) they have $(t, 3 x)$ confinement with $t \in \Omega(d)$;

(iii) for every small error $|e| \leq 3, \sigma(e)>1$.

Property (i) is true by assumption made by the authors in Ref. [20]. In order to prove property (ii) we use Corollary 9 of Ref. [20], which states that the code family considered has robustness. Robustness for a code is very similar to confinement but uses a slightly different notion of reduced weight, which, for an operator $e$, is defined as

$$
|e|_{\mathcal{S}}^{\text {red }}:=\min \{|e+s|: s \text { is a stabilizer }\} .
$$

Our definition of reduced weight instead minimizes over all Pauli operators with the same syndrome and therefore it considers both stabilizers and logical operators. Because for the reduced weight we minimize over a bigger set, the following holds:

$$
|e|_{\mathcal{S}}^{\mathrm{red}} \geq|e|^{\mathrm{red}}
$$

Confinement follows combining the statement of Corollary 9 in Ref. [20] for errors such that $|e|_{\mathcal{S}}^{\text {red }}<d$ and Eq. (D1):

$$
3|\sigma(e)| \geq|e|^{\mathrm{red}}
$$

In order to prove property (iii) we need to make use of the hypergraph product structure of the expander codes in Ref. [20]. Briefly, the code family is defined by taking the two-product of the length-1 chain complex $\delta: C_{0} \longrightarrow$ $C_{1}$ with itself, where $\delta$ is an expander matrix (see also Ref. [70]). More precisely, the expander codes in Ref. [20] are CSS codes defined on the chain complex:
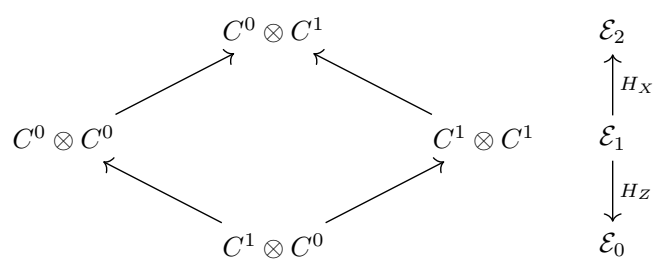

where

$$
\begin{aligned}
& H_{Z}=\left(\begin{array}{ll}
\delta \otimes \mathbb{1} & \mathbb{1} \otimes \delta^{T}
\end{array}\right), \\
& H_{X}=\left(\begin{array}{ll}
\mathbb{1} \otimes \delta & \delta^{T} \otimes \mathbb{1}
\end{array}\right) .
\end{aligned}
$$

The matrix $\delta$ is chosen in a family of LDPC expander matrices with full rank and constant column and row weight $w_{c}$ and $w_{r}$ bigger than two.

We prove property (iii) for $X$ errors $e$ and the syndrome map $\sigma(e)=H_{Z} \bar{e}$, where $\bar{e}$ is the binary vector representation of the Pauli operator $e$; the proof for $Z$ errors and syndrome map $H_{X}$ follows by duality with minor changes.

Let $e$ be a weight- $1 X$ operator and $\bar{e}$ its representation as a unit vector. Then $\sigma(e)=H_{Z} \bar{e}$ is a column of the matrix $H_{Z}$, namely column $j$ if $\bar{e}$ has $j$ th coordinate equal to 1 . Since the seed matrix $\delta$ has constant column and row degree bigger than 2, so has the matrix $H_{Z}$ and therefore $|\sigma(e)| \geq 2$.

Consider now a weight- 2 error operator $e$. By reshaping of vectors into matrices we can write $e$ as

$$
(L, R)
$$

for some binary matrices $L$ of size $n \times n, R$ of size $m \times$ $m$, and such that $|L|+|R|=2$, where $\delta$ has size $m \times n$. Following this notation, the syndrome $S$ of $E$ can be written as

$$
S=\delta L+R \delta
$$

We have three cases to be distinguished.

(a) $|L|=2$. If the support of $L$ is not contained in one column, i.e., $L_{i_{1}, j_{1}}=L_{i_{2}, j_{2}}=1$ and $j_{1} \neq j_{2}$, then for the syndrome $S=\delta L$ the following holds:

$$
S^{i_{1}}=\delta^{i_{1}} \text { and } S^{j_{2}}=\delta^{i_{2}}
$$

i.e., the syndrome matrix $S$ has at least two nonzero columns and therefore weight at least 2 . If instead the support of $L$ is contained in one column, $L_{i_{1}, j}=$ 
$L_{i_{2}, j} \neq 0$ then the syndrome $S$ is zero but for the $j$ th column:

$$
S^{j}=\delta^{i_{1}}+\delta^{i_{2}}
$$

In this case, whenever $\delta$ has distance at least 3, because it has constant column weight $w_{c}$, the following holds:

$$
\begin{aligned}
\left|\delta^{i_{1}}+\delta^{i_{2}}\right| & =\left|\delta^{i_{1}}\right|+\left|\delta^{i_{2}}\right|-2\left|\delta^{i_{1}} \wedge \delta^{i_{2}}\right| \\
& \geq 2 w_{c}-2\left(w_{c}-1\right) \\
& \geq 2 .
\end{aligned}
$$

Where the second to last inequality holds because if $\delta$ defines code of distance bigger than 3 , then it must have all distinct columns and different vectors of constant weight $w_{c}$ overlap in at most $w_{c}-1$ positions.

(b) $|L|=|R|=1$. Suppose $L_{i_{1}, j_{1}}=R_{i_{2}, j_{2}}=1$. The syndrome $S$ has support contained in one column and one row, in the shape of a cross as follows:

$$
\begin{array}{rlrl}
S_{k, j_{1}} & =\delta_{k, i_{1}}, & k \neq i_{2} \\
S_{i_{2}, k} & =\delta_{j_{2}, k}, & k \neq j_{1} \\
S_{i_{2}, j_{1}} & =\delta_{i_{2}, i_{1}}+\delta_{j_{2}, j_{1}}, & & \\
S_{i j} & =0, & & \text { otherwise. }
\end{array}
$$

It then follows for the weight of the syndrome $S$ that

$$
|S| \geq w_{c}+w_{r}-1
$$

which is bigger that 1 by assumption on the column and row weight of $\delta$.

(c) The case $|R|=2$ can be proven as done in (a) for $|L|=2$ by exchanging the role of columns and rows.

To sum up, whenever $|e|=2,|\sigma(e)|>2$.

Last, consider a weight-3 error $e$. As done for weight-2 errors, we can write $e$ as $(L, R)$ for some binary matrices $L$ and $R$. Again, we need to distinguish between the possible weight combinations of $|L|+|R|=3$. We now prove (a)

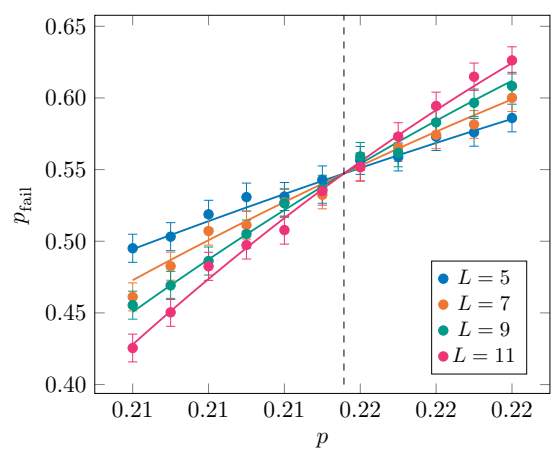

(d)

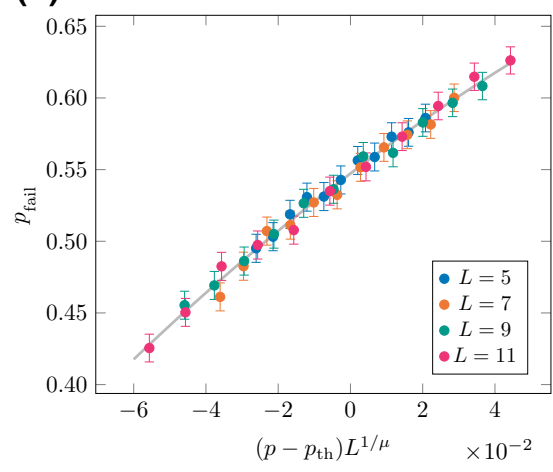

(b)

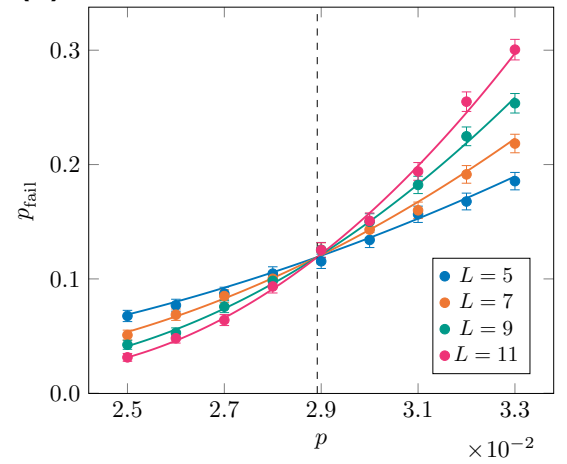

(e)

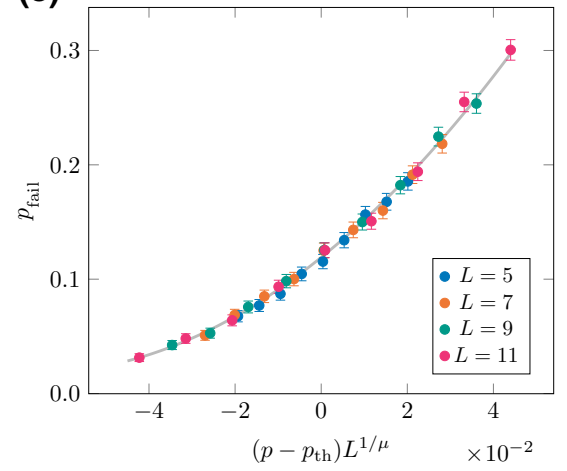

(c)

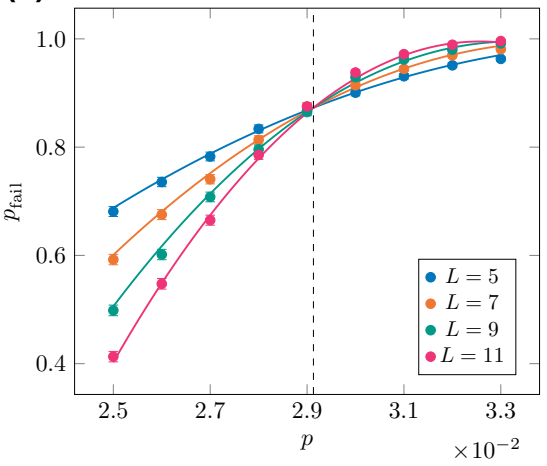

(f)

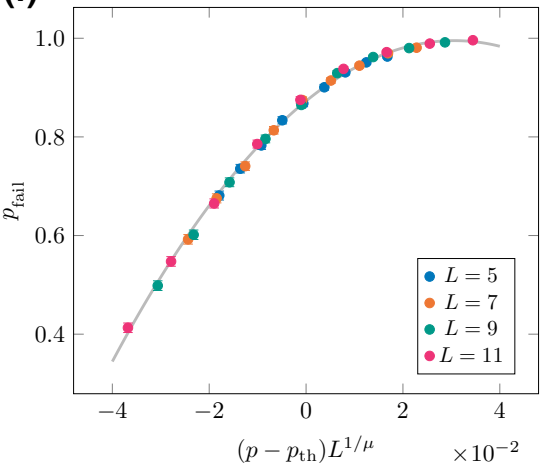

FIG. 9. Threshold fits for the 3D toric code using MWPM and BP+OSD to decode. In (a), we plot the logical error rate $p_{\text {fail }}$ as a function of the phase-flip error rate $p$, for values of $p$ close to the threshold. The colored lines show the fit given by Eq. (E1), with parameters $a_{0}=0.547, a_{1}=1.92, a_{2}=-4.04, \mu=1.04$, and $p_{\text {th }}=0.216$ (dashed gray line). In (d), we show the same data using the rescaled variable $x=\left(p-p_{\text {th }}\right) L^{1 / \mu}$. Subfigures (b) and (e) show equivalent data for one round of single-shot error correction, with fit parameters $a_{0}=0.119, a_{1}=3.04, a_{2}=22.9, \mu=1.01$, and $p_{\text {th }}=0.0289$. Subfigures (c) and (f) show equivalent data for 16 rounds of single-shot error correction, with fit parameters $a_{0}=0.873, a_{1}=7.99, a_{2}=-130, \mu=1.10$, and $p_{\text {th }}=0.0291$. The error bars show the $95 \%$ confidence intervals $p_{\text {fail }}=\hat{p}_{\text {fail }} \pm 1.96 \sqrt{p_{\text {fail }}\left(1-p_{\text {fail }}\right) / \eta}$, where $\eta \geq 10^{4}$ is the number of Monte Carlo trials. 
the case for $|L|=3$ and support of $L$ contained in one column. The other cases are either a dual argument of this one (i.e., for $|R|=3$ supported on one row) or follows easily adapting the proof for $|e|=2$.

Let $e$ be a weight-3 error operator with reshaping $(L, R)$ such that $|L|=3$ and $L_{i_{1}, j}=L_{i_{2}, j}=L_{i_{3}, j}=1$, for some column index $j$. In this case, the syndrome matrix $S$ has support contained in its $j$ th column:

$$
S^{j}=\delta^{i_{1}}+\delta^{i_{2}}+\delta^{i_{3}}
$$

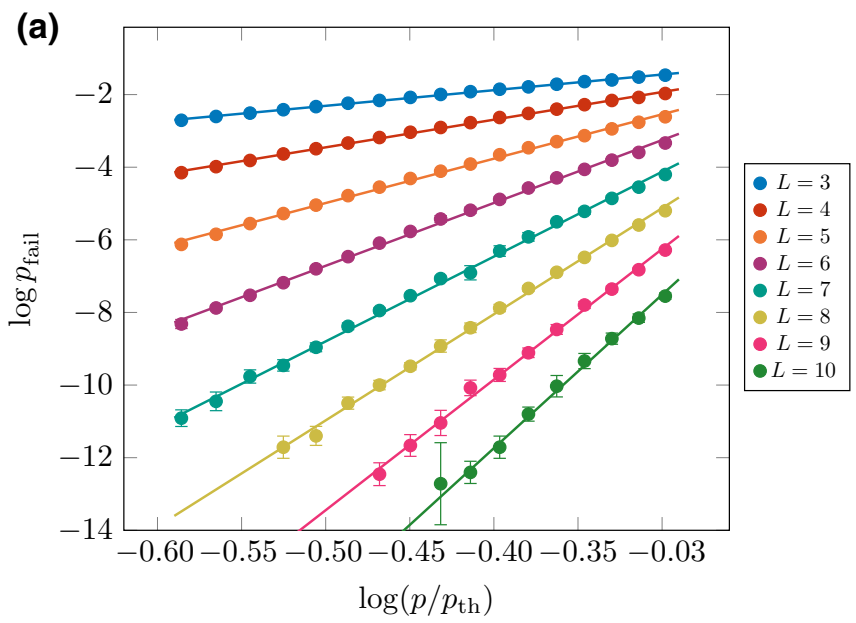

(c)

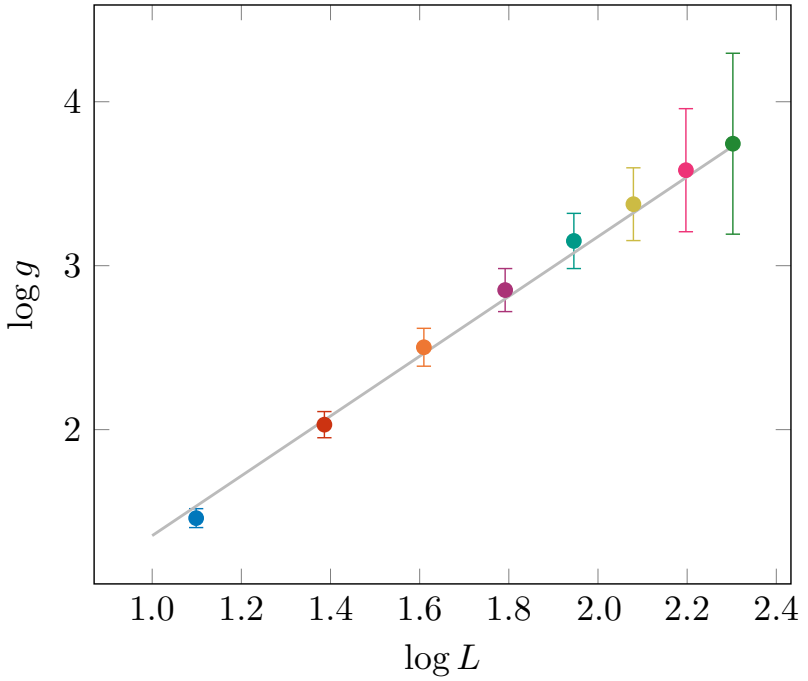

and therefore,

$$
|\sigma(e)|=|S|=\left|S^{j}\right|=\left|\delta^{i_{1}}+\delta^{i_{2}}+\delta^{i_{3}}\right| .
$$

In order to prove $|\sigma(e)|=\left|S^{j}\right|>2$, we need to use the expansion properties of $\delta$ and more specifically Lemma 3 of Ref. [20], (see also Ref. [70]). We first introduce some notation. We refer to the rows of $\delta$ as checks and to its columns as bits; we say that a bit $j$ is in the support of the check $i$ if and only if $\delta_{i j}=1$. Given a set of bits $B \subseteq\{1, \ldots, n\}$ we say that the check $i \in\{1, \ldots, m\}$

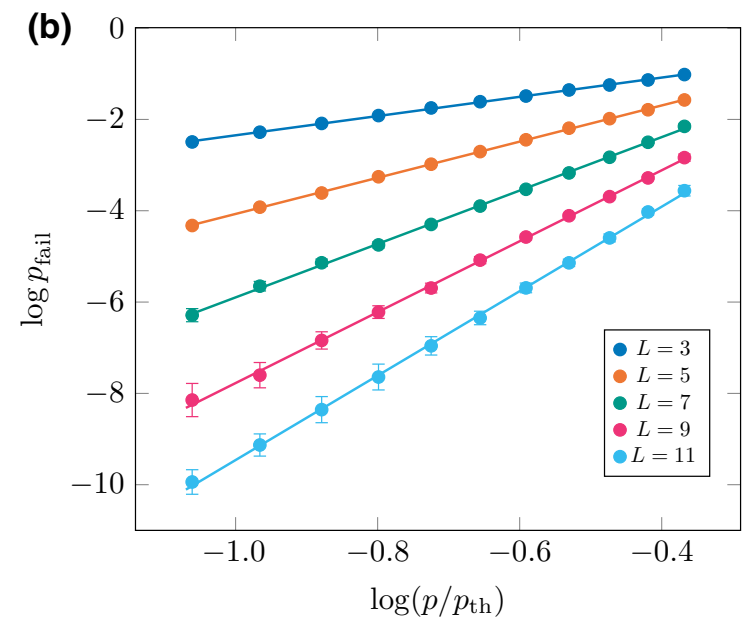

(d)

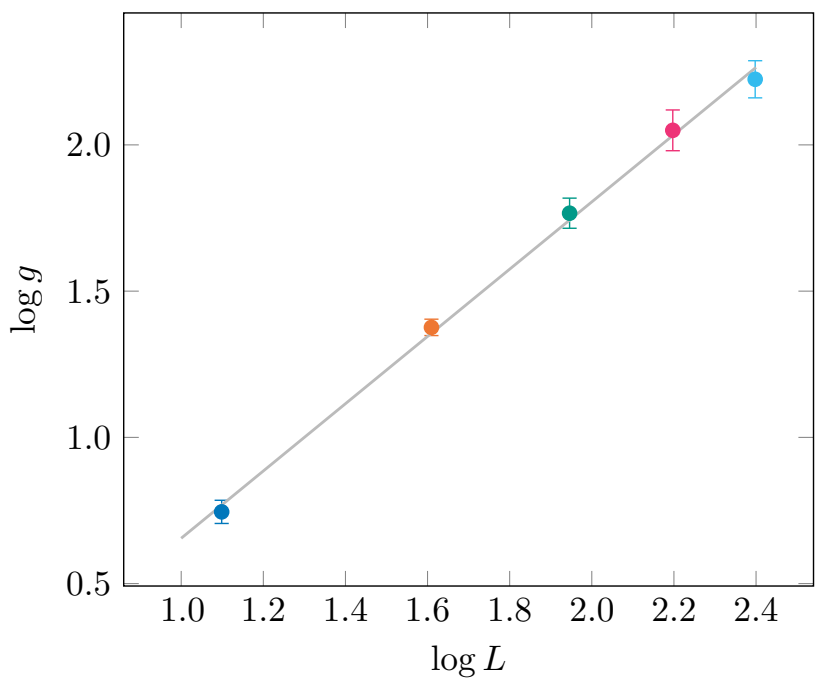

FIG. 10. Illustration of the fitting procedure for finding the coefficients describing the suppression of the logical error rate for phaseflip error rates substantially below threshold. (a),(c) Data for code-capacity noise (no measurement errors), and (b),(d) show data for eight rounds of single-shot error correction. In both cases, we first plot $\log p_{\text {fail }}$ as a function of $\log \left(p / p_{\text {th }}\right)$ for differing values of $L$, observing trends that agree with the straight line prediction of Eq. (E3) [(a),(b)]. We note that for the single-shot case there is an odd-even effect so we include only the data for odd $L$. We extract the gradients $g(L)$ from the corresponding straight line fits in (a),(b) (gray lines), and plot the logarithms of these values against $\log L[(\mathrm{c}),(\mathrm{d})]$. The data fit well to the linear ansatz given in Eq. (E5), which allows us to estimate the parameters $\alpha$ and $\beta$, which control the suppression of the logical error rate as per Eq. (E2). For code-capacity noise, we estimate $\alpha=0.546(33)$ and $\beta=1.91(3)$, and for eight rounds of single-shot error correction, we estimate $\alpha=0.610(37)$ and $\beta=1.15$ (3). The error bars in (a),(b) show the $95 \%$ confidence intervals $\log p_{\text {fail }}=\log \hat{p}_{\text {fail }} \pm \frac{1.96}{p_{\text {fail }}} \sqrt{p_{\text {fail }}\left(1-p_{\text {fail }}\right) / \eta}$, where $\eta \geq 10^{4}$ is the number of Monte Carlo trials. We include data points with at least 25 failures. The error bars in (c),(d) show the $95 \%$ confidence intervals given by the LINEARMODELFIT function of Mathematica. 
is a unique neighbor of $B$ if and only if one and only one bit in $B$ belongs to the support of the check $i$. We indicate with $\Gamma_{u}(B)$ the set of unique neighbors of $B$. Lemma 3 in Ref. [20] states that, for the considered class of matrices $\delta$ :

$$
\left|\Gamma_{u}(B)\right| \geq \frac{2}{3} w_{c}|B| .
$$

Combining this with the observation that $\left|\Gamma_{u}\left(\left\{i_{1}, i_{2}, i_{3}\right\}\right)\right|$ is a lower bound on $\left|S^{j}\right|$ and plugging in $|B|=3$, we find

$$
\left|S^{j}\right| \geq\left|\Gamma_{u}\left(\left\{i_{1}, i_{2}, i_{3}\right\}\right)\right| \geq 2 w_{c} .
$$

To sum up, whenever an error $(L, R)$ of weight 3 has support on either one column of $L$ or one row of $R$, by expansion its syndrome has weight strictly bigger than 1 . When instead a weight-3 error has support spread among more than one column and row it is enough to use the hypergraph product structure of the code family, as done for weight-2 errors, to find that its syndrome need to have weight at least 2 .

\section{APPENDIX E: FITTING DETAILS}

To obtain our threshold estimates, we use the standard critical exponent method [59]. Specifically, in the vicinity of the threshold, we fit our data to the following ansatz:

$$
a_{0}+a_{1} x+a_{2} x^{2}
$$

where the rescaled variable $x=\left(p-p_{\mathrm{t} h}\right) L^{1 / \mu}$. Examples of this fit are shown in Fig. 9.

We use the fitting method described in Ref. [12] to understand the behavior of the 3D toric code logical error rate for error rates $p$ significantly below threshold. Recall from Sec. V that we use the following ansatz:

$$
p_{\text {fail }}(L) \propto\left(p / p_{\text {th }}\right)^{\alpha L^{\beta}},
$$

we take the logarithm of both sides to obtain

$$
\log p_{\text {fail }}=\log f(L)+\alpha L^{\beta} \log \left(p / p_{\text {th }}\right) .
$$

For different values of $L$, we plot $\log p_{\text {fail }}$ as a function of $\log \left(p / p_{\text {th }}\right)$ and fit to a straight line to obtain gradients

$$
g(L)=\frac{\partial \log p_{\text {fail }}}{\partial u}=\alpha L^{\beta},
$$

where $u=\log \left(p / p_{\text {th }}\right)$. Finally, take the logarithm of both sides of the above to give

$$
\log g=\log \alpha+\beta \log L
$$

We then plot $\log g$ as a function of $\log L$ and fit to a straight line to get $\alpha$ and $\beta$. Figure 10 illustrates the above fitting procedure for code-capacity noise (no measurement errors) and for eight rounds of single-shot error correction.

[1] Joschka Roffe, Quantum error correction: An introductory guide, Contemporary Phys. 60, 226 (2019).

[2] Eric Dennis, Alexei Kitaev, Andrew Landahl, and John Preskill, Topological quantum memory, J. Math. Phys. 43, 4452 (2002).

[3] Austin G. Fowler, Ashley M. Stephens, and Peter Groszkowski, High-threshold universal quantum computation on the surface code, Phys. Rev. A 80, 052312 (2009).

[4] R. Raussendorf, J. Harrington, and K. Goyal, Topological fault-tolerance in cluster state quantum computation, New J. Phys. 9, 199 (2007).

[5] A. Bolt, G. Duclos-Cianci, D. Poulin, and T. M. Stace, Foliated Quantum Error-Correcting Codes, Phys. Rev. Lett. 117, 070501 (2016).

[6] Naomi Nickerson and Héctor Bombín, Measurement based fault tolerance beyond foliation, arXiv:1810.09621 (2018).

[7] Hector Bombin, 2D quantum computation with 3D topological codes, arXiv:1810.09571 (2018).

[8] Benjamin J. Brown, A fault-tolerant non-Clifford gate for the surface code in two dimensions, arXiv:1903.11634 (2019).

[9] Michael Newman, Leonardo Andreta de Castro, and Kenneth R. Brown, Generating fault-tolerant cluster states from crystal structures, arXiv:1909.11817 (2019).

[10] Héctor Bombín, Single-Shot Fault-Tolerant Quantum Error Correction, Phys. Rev. X 5, 031043 (2015).

[11] Héctor Bombín, Resilience to Time-Correlated Noise in Quantum Computation, Phys. Rev. X 6, 041034 (2016).

[12] Benjamin J. Brown, Naomi H. Nickerson, and Dan E. Browne, Fault-tolerant error correction with the gauge color code, Nat. Commun. 7, 1 (2016).

[13] Kasper Duivenvoorden, Nikolas P. Breuckmann, and Barbara M. Terhal, Renormalization group decoder for a fourdimensional toric code, IEEE Trans. Inf. Theory 65, 2545 (2018).

[14] Nikolas P. Breuckmann and Vivien Londe, Single-shot decoding of linear rate LDPC quantum codes with high performance, arXiv:2001.03568 (2020).

[15] Aleksander Kubica, Ph.D. thesis, Caltech, 2018.

[16] Aleksander Kubica and John Preskill, Cellular-Automaton Decoders with Provable Thresholds for Topological Codes, Phys. Rev. Lett. 123, 020501 (2019).

[17] Michael Vasmer, Dan E. Browne, and Aleksander Kubica, Cellular automaton decoders for topological quantum codes with noisy measurements and beyond, arXiv:2004.07247 (2020).

[18] Earl T. Campbell, A theory of single-shot error correction for adversarial noise, Quantum Sci. Technol. 4, 025006 (2019).

[19] Jean-Pierre Tillich and Gilles Zémor, Quantum LDPC codes with positive rate and minimum distance proportional to the square root of the blocklength, IEEE Trans. Inf. Theory 60, 1193 (2014).

[20] Anthony Leverrier, Jean-Pierre Tillich, and Gilles Zémor, in Foundations of Computer Science (FOCS), 2015 IEEE 56th Annual Symposium on (IEEE, 2015), p. 810. 
[21] Omar Fawzi, Antoine Grospellier, and Anthony Leverrier, in Proc. STOC (ACM, 2018), p. 521.

[22] Omar Fawzi, Antoine Grospellier, and Anthony Leverrier, Constant overhead quantum fault tolerance with quantum expander codes. to appear in FOCS 2018, (2018).

[23] Austin G. Fowler, Time-optimal quantum computation, arXiv:1210.4626 (2012).

[24] Barbara M. Terhal, Quantum error correction for quantum memories, Rev. Mod. Phys. 87, 307 (2015).

[25] Poulami Das, Christopher A. Pattison, Srilatha Manne, Douglas Carmean, Krysta Svore, Moinuddin Qureshi, and Nicolas Delfosse, A Scalable Decoder Micro-architecture for Fault-Tolerant Quantum Computing, arXiv:2001.06598 (2020), p. 1.

[26] Antoine Grospellier and Anirudh Krishna, Numerical study of hypergraph product codes, arXiv:1810.03681 (2018).

[27] Antoine Grospellier, Lucien Grouès, Anirudh Krishna, and Anthony Leverrier, Combining hard and soft decoders for hypergraph product codes, arXiv:2004.11199 (2020).

[28] Sergey Bravyi and Matthew B. Hastings, in Proceedings of the 46th Annual ACM Symposium on Theory of Computing (ACM, 2014), p. 273.

[29] Benjamin Audoux and Alain Couvreur, On tensor products of CSS codes, arXiv:1512.07081 (2015).

[30] Arun B. Aloshious and Pradeep Kiran Sarvepalli, Decoding toric codes on three dimensional simplical complexes, arXiv:1911.06056 (2019).

[31] Nikolas P. Breuckmann and Xiaotong Ni, Scalable neural network decoders for higher dimensional quantum codes, Quantum 2, 68 (2018).

[32] Yukiyasu Ozeki and Nobuyasu Ito, Multicritical dynamics for the $\pm \mathrm{J}$ Ising model, J. Phys. A: Math. General 31, 5451 (1998).

[33] Takuya Ohno, Gaku Arakawa, Ikuo Ichinose, and Tetsuo Matsui, Phase structure of the random-plaquette $\mathbb{Z}_{2}$ gauge model: Accuracy threshold for a toric quantum memory, Nucl. Phys. B 697, 462 (2004).

[34] Martin Hasenbusch, Francesco Parisen Toldin, Andrea Pelissetto, and Ettore Vicari, Magnetic-glassy multicritical behavior of the three-dimensional $\pm \mathrm{J}$ Ising model, Phys. Rev. B - Condens. Matter Mater. Phys. 76, 184202 (2007).

[35] Koujin Takeda and Hidetoshi Nishimori, Self-dual randomplaquette gauge model and the quantum toric code, Nucl. Phys. B 686, 377 (2004).

[36] Aleksander Kubica, Michael E. Beverland, Fernando Brandão, John Preskill, and Krysta M. Svore, ThreeDimensional Color Code Thresholds via StatisticalMechanical Mapping, Phys. Rev. Lett. 120, 180501 (2018).

[37] Alexey A. Kovalev and Leonid P. Pryadko, Fault tolerance of quantum low-density parity check codes with sublinear distance scaling, Phys. Rev. A 87, 020304 (2013).

[38] Sergey Bravyi and Matthew B. Hastings, in Proceedings of the forty-sixth annual ACM symposium on Theory of computing (ACM, 2014), p. 273.

[39] Allen Hatcher, Algebraic Topology. 2002 (Cambridge UP, Cambridge, 2002), p. 606.

[40] Weilei Zeng and Leonid P. Pryadko, Higher-Dimensional Quantum Hypergraph-Product Codes with Finite Rates, Phys. Rev. Lett. 122, 230501 (2019).

[41] Jack Edmonds, Paths, trees, and flowers, Canadian J. Math. 17, 449 (1965).
[42] Austin G. Fowler, Matteo Mariantoni, John M. Martinis, and Andrew N. Cleland, Surface codes: Towards practical large-scale quantum computation, Phys. Rev. A 86, 032324 (2012).

[43] Nikolas P. Breuckmann and Barbara M. Terhal, Constructions and noise threshold of hyperbolic surface codes, IEEE Trans. Inf. Theory 62, 3731 (2016).

[44] Benjamin J. Brown and Dominic J. Williamson, Parallelized quantum error correction with fracton topological codes, Phys. Rev. Res. 2, 013303 (2020).

[45] Aleksander Kubica and Nicolas Delfosse, Efficient color code decoders in $d \geq 2$ dimensions from toric code decoders, arXiv:1905.07393 (2019).

[46] Namely, the dual lattice of the one described in Appendix B.

[47] Vladimir Kolmogorov, Blossom V: A new implementation of a minimum cost perfect matching algorithm, Math. Program. Comput. 1, 43 (2009).

[48] David J. C. MacKay and Radford M. Neal, Near shannon limit performance of low density parity check codes, Electron. Lett. 33, 457 (1997).

[49] F. R. Kschischang, B. J. Frey, and H. Loeliger, Factor graphs and the sum-product algorithm, IEEE Trans. Inf. Theory 47, 498 (2001).

[50] David Poulin and Yeojin Chung, On the iterative decoding of sparse quantum codes, Quantum Info. Comput. 8, 987 (November 2008).

[51] Z. Babar, P. Botsinis, D. Alanis, S. X. Ng, and L. Hanzo, Fifteen years of quantum ldpc coding and improved decoding strategies, IEEE Access 3, 2492 (2015).

[52] Ye-Hua Liu and David Poulin, Neural Belief-Propagation Decoders for Quantum Error-Correcting Codes, Phys. Rev. Lett. 122, 200501 (2019).

[53] Alex Rigby, J. C. Olivier, and Peter Jarvis, Modified belief propagation decoders for quantum low-density paritycheck codes, Phys. Rev. A 100, 012330 (Jul 2019).

[54] M. Li and T. J. Yoder, in 2020 IEEE International Conference on Quantum Computing and Engineering (QCE) (2020), p. 109.

[55] Pavel Panteleev and Gleb Kalachev, Degenerate quantum LDPC codes with good finite length performance, arXiv:1904.02703 (2019).

[56] Joschka Roffe, David R. White, Simon Burton, and Earl Campbell, Decoding across the quantum low-density parity-check code landscape, Phys. Rev. Res. 2, 043423 (Dec 2020).

[57] Joschka Roffe, BP+OSD - a decoder for sparse quantum codes. github.com.

[58] More precisely, rows of $L_{M}$ are vectors in $\mathcal{C}_{2}$ that correspond to elements of the second cohomology group $\mathcal{H}_{2}^{*}$; hence their weight is lower bounded by $d_{2}^{*}=$ $\min \left\{d_{a}^{T} d_{b}^{T}, d_{a}^{T} d_{c}^{T}, d_{b}^{T} d_{c}^{T}\right\}$, see Ref. [40].

[59] J. Harrington, Ph.D. thesis, Caltech, 2004.

[60] Chenyang Wang, Jim Harrington, and John Preskill, Confinement-higgs transition in a disordered gauge theory and the accuracy threshold for quantum memory, Ann. Phys. 303, 31 (2003).

[61] Sagar Vijay, Jeongwan Haah, and Liang Fu, Fracton topological order, generalized lattice gauge theory, and duality, Phys. Rev. B 94, 235157 (2016).

[62] Arpit Dua, Isaac H. Kim, Meng Cheng, and Dominic J. Williamson, Sorting topological stabilizer models 
in three dimensions, Phys. Rev. B 100, 155137 (2019).

[63] www.computeontario.ca.

[64] www.computecanada.ca.

[65] Antoine Grospellier, Ph.D. thesis, Sorbonne universités, 2019.

[66] When the code is a CSS code we consider the group generated by the $X$ stabilizers and the $Z$ stabilizers separately. $\mathcal{S}$ will thus refer either to $\mathcal{S}_{X}$ or $\mathcal{S}_{Z}$.
[67] For example, if $e_{1}$ and $e_{2}$ are both $X$ operators, $E_{1}+E_{2}$ is the symmetric difference of the sets $E_{1}$ and $E_{2}$.

[68] $\left(\begin{array}{l}n \\ k\end{array}\right) \simeq 2^{n h(k / n)}$, where $h(x)=x \log _{2}(1 / x)+(1-x) \log _{2}$ $[1 /(1-x)]$ is the binary entropy function.

[69] A real function is said subadditive if for any $x, y \in \mathbb{R}$, $g(x)+g(y) \geq g(x+y)$.

[70] Michael Sipser and Daniel A. Spielman, Expander codes, IEEE Trans. Inf. Theory 42, 1710 (1996). 